\title{
Surge Instability on a Cavitating Propeller
}

\author{
Thesis by \\ Mark Edward Duttweiler
}

In Partial Fulfillment of the Requirements

for the Degree of

Doctor of Philosophy

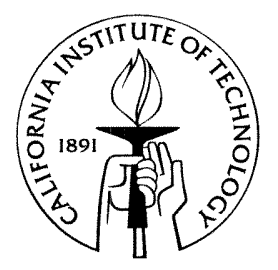

California Institute of Technology

Pasadena, California

2001

(Defended September 26, 2000) 
(c) 2001

Mark Edward Duttweiler

All rights Reserved 


\section{Acknowledgements}

The ensuing attempt to encapsulate five years of research in a relatively short and concise document implies that my experiences at Caltech have been limited to a brief endeavor into fluid mechanics insulated within the laboratory. In fact, it has been so much more. This is due in large part to the wealth of fantastically bright, delightfully supportive individuals throughout the Caltech community to whom I wish to express my sincerest gratitude.

Above all, I thank my advisor, Professor Christopher Brennen. I am privileged to have had the opportunity to learn from such an accomplished and respected individual the wonders of research. His careful guidance and support educated me in not only a small corner of scientific inquiry, but in entire methods of thought and exploration previously foreign to me.

I also appreciated the continued support and constructive suggestions offered by the other members of my thesis committee, Professors Alan Acosta, Fred Raichlen, Richard Murray, and Tim Colonius. Essential as well were the generous support of the Office of Naval Research, which supported this work under Contract N-00014-97-1-0002, and the oversight of technical monitor Dr. Edwin Rood. Dr. Stuart Jessup of the David Taylor Model Basin was kind enough to provide the model Navy propeller used in the experiments.

I am also indebted to the graduate students who have come and gone before me. I thank Dr. Garrett Reisman for exhibiting extraordinary patience while enlightening me in the subtle moods of the Low Turbulence Water Tunnel, and for imparting to me the courage needed to manage such a facility. Dr. Tricia Sur also provided much guidance and advice regarding many of the experimental methods used in the research. Drs. Elizabeth McKenney and Douglas Hart labored to design and construct much of the experimental equipment that performed so reliably throughout this work.

Clancy Rowley, from the very beginning, offered continued friendship and kind words, especially through the tense moments leading up to our candidacy examinations. In the following years, Al Preston accompanied me to high and remote places where adrenaline and fear convinced me that enclosing myself within the lab seemed like a prudent idea, if only for safety. Throughout the course of the research, several undergraduate students provided invaluable assistance in conducting many of the necessary experiments. Don Kwak, Phil Rodriguez, Rajat Kongovi, Dale Parkes, Russel Howe, and especially Steven Schell provided not only extra hands, but motivation, insight, and friendship as well.

I thank Rodney Rojas and John Van Deusen for introducing me to the wonders and joys of machining. When it seemed there was no way to make this from that, they showed me how. Rich Eastvedt and Mike Vondrus also offered valuable support in maintaining and improving the Low Turbulence Water Tunnel facility. Countless were the times when Dana Young delivered sincere responses to my inane questions or calming words to my uneasy mind. My thanks also to Dr. Andrew Lewis, who developed the citthesis $\mathrm{LT}_{\mathrm{E}} \mathrm{X}$ document class, and in so doing spared myself and others numerous hours of struggle.

A very special thank you also to Waterman, Baden Powell, and San Antonio. These places were a continued source of inspiration during some of the lower moments. Upon arrival at Caltech, I had the great fortune of meeting in Stuart Baur a longtime friend 
whose thoughts and support have also been appreciated. To the members of Ruddock House, I offer my gratitude, for extending my Caltech experience beyond what I had imagined possible, and for providing me with countless cherished memories.

Lastly, I thank my father, for introducing me to the wonders of science and engineering at an early age; my brother, for showing me the way; my mother, for always believing; and Christine, for holding my hand and my heart with such care and patience. 


\title{
Surge Instability on a Cavitating Propeller \\ by
}

\author{
Mark Edward Duttweiler
}

In Partial Fulfillment of the

Requirements for the Degree of

Doctor of Philosophy

\section{Abstract}

The present study details results from experiments investigating a surge instability on a cavitating propeller. Initially, the stable behavior of the propeller is explored, and the nature and extent of the cavitation is documented at various experimental conditions, including propeller yaw. The cavitation surge instability is first explored through visual observation of the cavitation on the propeller blades and in the tip vortices. Particular note is made of similarities between the behavior of the re-entrant jets and that noted by other investigators. It is also observed that the nature of the instability is closely related to the partial cavity instability observed on single, two-dimensional hydrofoils.

The flow conditions that lead to instability are determined and it is shown that onset corresponds to a specific configuration of attached cavity lengths on an individual propeller blade. Pressure measurements are obtained from transducers within the experimental facility, and the acoustic signature of the instability is identified. The magnitude of the fluctuating pressures is very large, presumably capable of producing severe hull vibration. A simple model is developed based on cavity volume estimates obtained from high speed video footage, and the predictions of the model are compared with the experimentally obtained pressures.

To assess the significance of the surrounding facility in initiating and sustaining the instability, a model is developed for the experimental facility dynamics. The predictions of this model are then compared with an experimentally determined facility response to a volumetric excitation imposed by an oscillating piston. To quantify the response of the 
cavitation to fluctuations in test section conditions, quasistatic estimates are obtained for the cavitation compliance and mass flow gain factor of the propeller. These parameters have previously been employed in developing system transfer functions for cavitating pumps.

Finally, a model is developed for the complete system, incorporating both the cavitation and facility dynamics. The model predicts active system dynamics and therefore potentially unstable behavior for two distinct frequency ranges, and one such range is hypothesized to correspond to the observed instability. The ability of the model to predict the observed characteristics of the instability is then evaluated. 


\section{Contents}

1 Introduction 1

1.1 Adverse Effects of Cavitation . . . . . . . . . . . . . . . 1

1.2 Unsteady Cavitation Studies . . . . . . . . . . . . . . . 2

1.2.1 Cloud Cavitation . . . . . . . . . . . . . . . . 2

1.2 .2 Hydrofoils . . . . . . . . . . . . . . . . . . . 2

$1.2 .3 \quad$ Pumps . . . . . . . . . . . . . . . . . 3

1.2 .4 Propellers . . . . . . . . . . . . . . . . . 4

1.3 Current Work . . . . . . . . . . . . . . . . . 5

2 Experimental Facilities and Methods $\quad 7$

2.1 Low Turbulence Water Tunnel Facility . . . . . . . . . . . . . . . . 7

2.2 Propeller Experiments . . . . . . . . . . . . . . . . . . . . . 9

2.2 .1 Propeller and Propeller Drive . . . . . . . . . . . . . . . . 9

2.2 .2 Pressure Measurements . . . . . . . . . . . . . . . . . 11

2.2 .3 Visual Observations . . . . . . . . . . . . . . . . 12

2.3 Oscillating Piston Experiments . . . . . . . . . . . . . . . 14

2.3.1 Oscillating Piston Mechanism . . . . . . . . . . . . . 14

2.3 .2 Pressure Measurements . . . . . . . . . . . . . 16

2.4 Data Acquisition and Processing . . . . . . . . . . . . . . 16

3 Cavitating Propeller Experiments $\quad 17$

3.1 Experimental Parameters . . . . . . . . . . . . . . . . . . . . . 17

3.2 Stable Cavitating Behavior . . . . . . . . . . . . . . . . . . 19 
3.2 .1 Propeller at Zero Yaw . . . . . . . . . . . . . . . . . 19

3.2 .2 Propeller at Yaw . . . . . . . . . . . . . . 2 25

3.3 Unstable Cavitating Behavior . . . . . . . . . . . . . . . 27

3.3.1 General Description of the Instability . . . . . . . . . . 27

3.3.2 Re-entrant Jet Behavior on Swept Hydrofoils . . . . . . . . . . 28

3.3.3 Description of the Instability Cycle . . . . . . . . . . . 30

3.3 .4 Similarities to the Partial Cavity Instability . . . . . . . . . . 31

3.3.5 Measurement of the Pressures Generated by the Instability . . . . 32

3.3 .6 Onset of the Instability f . . . . . . . . . . . . . . 34

3.3.7 Variation of the Instability Frequency . . . . . . . . . . 37

3.3.8 Analysis of the Pressures Generated by the Instability . . . . . . . 40

4 Experimental Facility Dynamics $\quad 46$

4.1 Modeling of the Facility Dynamics . . . . . . . . . . . . . 46

4.1 .1 System Impedance . . . . . . . . . . . . . . . . . 46

$4.1 .2 \quad$ Lumped Parameters . . . . . . . . . . . . . . . . . . . 48

4.1.3 Characterization of the Experimental Facility . . . . . . . . . 50

4.1.4 Evaluation of the Lumped Parameters . . . . . . . . . . . . 52

4.2 Natural Frequency Experiments . . . . . . . . . . . . . . . . 59

4.2.1 Prediction and Measurement of the Natural Frequency . . . . . . 59

4.2 .2 Effect of Overflow Tank Conditions . . . . . . . . . . . . 61

4.2 .3 Effect of Air on Tunnel Compliance . . . . . . . . . . . . . . 64

4.3 Forced Oscillation Experiments . . . . . . . . . . . . . . . . . 67

4.3.1 Experimental Determination of the Facility Response . . . . . . 67

4.3.2 Magnitude of Response . . . . . . . . . . . . . . . 69

4.3 .3 Phase of Response . . . . . . . . . . . . . . . . 72

4.3 .4 Test Section Inertance . . . . . . . . . . . . . . . . 74

4.3 .5 Resistances . . . . . . . . . . . . . . 76

4.3.6 Effect of Mean Flow . . . . . . . . . . . . . . . 77

4.4 Far Field Pressure Attenuation . . . . . . . . . . . . . . 78 
5 Cavitation Dynamics $\quad \mathbf{8 0}$

5.1 Development of Cavitation Dynamic Parameters . . . . . . . . . . . . 80

5.2 Estimation of Cavitation Dynamic Parameters . . . . . . . . . . . 81

5.3 Comparison of Cavitation Dynamic Parameters . . . . . . . . . . . . 85

5.4 Effect of Tip Vortex Participation _. . . . . . . . . . . 87

6 Combined Facility and Cavitation Dynamics $\quad 89$

6.1 Passive and Active Dynamics _ . . . . . . . . . . . . . . . 89

6.1 .1 System Impedance $\ldots \ldots \ldots$. . . . . . . . . . . . . 89

6.1 .2 Fluctuation Energy Flux . . . . . . . . . . . . . . . . . 91

6.2 Local Impedance . . . . . . . . . . . . . . . . . . . . . . . . . . 91

6.3 Modeling of the System . . . . . . . . . . . . . . . . . . . 93

$6.3 .1 \quad$ Full Model . . . . . . . . . . . . . . . . . . . . . . 93

6.3 .2 Simplified Model . . . . . . . . . . . . . . . . . 96

6.4 Effect of Tip Vortex Participation _ . . . . . . . . . . . . . 98

6.5 Variation of the Instability Frequency $\ldots \ldots \ldots \ldots$

6.6 Effect of Resistance . . . . . . . . . . . . . . . . . . . . . 100

6.7 Modeling of the Far Field Pressure . . . . . . . . . . . . . . . . 102

7 Conclusions $\quad 105$

7.1 Summary of Thesis Work . . . . . . . . . . . . . . . . . 105

7.2 Future Research . . . . . . . . . . . . . . . . . . . . 108 


\section{List of Figures}

2.1 Overview of Low Turbulence Water Tunnel facility. . . . . . . . . . . . 8

2.2 Propeller drive experimental setup. . . . . . . . . . . . . . . . 9

2.3 Propeller, propeller mount, and right angle gear case. . . . . . . . . . 10

2.4 Schematic of propeller drive installation and pressure transducer locations within tunnel test section. . . . . . . . . . . . . . . . . . 12

2.5 Oscillating piston experimental setup. . . . . . . . . . . 15

3.1 Effective angle of attack of propeller blade. . . . . . . . . . . 18

3.2 Photograph of stable propeller cavitation. . . . . . . . . . . 20

3.3 Schematic of cavitation features and cavitation extent. . . . . . . . . 20

3.4 Cavitation extent at low rotation speed. . . . . . . . . . . . . . . 22

3.5 Cavitation extent at moderate rotation speed. . . . . . . . . . . 23

3.6 Cavitation extent at high rotation speed. . . . . . . . . . . . . 24

3.7 Cavitation extent for yawed propeller. . . . . . . . . . 26

3.8 Photograph of re-entrant jet on three-dimensional hydrofoil. . . . . . . . 28

3.9 Schematic of re-entrant jet on three-dimensional hydrofoil. . . . . . . . . . 29

3.10 Schematic of reflection of re-entrant jet at a cavity closure. . . . . . . . 29

3.11 Cavitation variation during instability cycle. . . . . . . . . . . 31

3.12 Measured pressures generated by instability. . . . . . . . . . . . . 33

3.13 Power spectral density of pressures generated by instability. . . . . . . . 33

3.14 Series of power spectral densities at decreasing cavitation number. . . . . 35

3.15 Onset of instability with cavitation number and advance ratio. . . . . . 36

3.16 Comparison of instability onset with stability criterion based on dimensionless cavity length. . . . . . . . . . . . . . . 37 
3.17 Variation in instability frequency with advance ratio. . . . . . . . . . 39

3.18 Variation in instability frequency with cavitation number. . . . . . . . 39

3.19 Non-dimensional cavity length through several instability cycles. . . . . . 41

3.20 Comparison of volumetric acceleration and measured pressure. . . . . . . 43

3.21 Predicted fluctuating pressure at floor-mounted transducer. . . . . . . . . 44

4.1 Schematic of facility excitation and impedance. . . . . . . . . . . . 47

4.2 Lumped parameters utilized in experimental facility model. . . . . . . . 49

4.3 Significant features of experimental facility. . . . . . . . . . . 50

4.4 Impedance of lumped parameters. . . . . . . . . . . . . . . 51

4.5 Schematic of experimental facility. . . . . . . . . . . . 52

4.6 Experimentally measured deflection of tunnel walls. . . . . . . . . . . . 57

4.7 Location of tunnel wall deflection measurements. . . . . . . . . . . . . 58

4.8 Fluctuating pressure during natural frequency experiments. . . . . . . 61

4.9 Natural frequency with changing overflow tank free surface area. . . . . 63

4.10 Natural frequency with changing overflow tank pressure. . . . . . . . . 64

4.11 Effect of trapped air on natural frequency. . . . . . . . . . . . . 66

4.12 Effect of deaeration on natural frequency. . . . . . . . . . . 67

4.13 Fluctuating pressure during forced response experiments. . . . . . . . . 68

4.14 Magnitude of system impedance at low frequencies. . . . . . . . . . 70

4.15 Magnitude of system impedance at high frequencies. . . . . . . . . . 71

4.16 Phase of system impedance at low frequencies. . . . . . . . . . . . 73

4.17 Magnitude of system impedance calculated from ceiling-mounted and test section centerline transducer measurements. . . . . . . . . . 74

4.18 Effect of resistance on system impedance at low frequencies. . . . . . . 76

4.19 Effect of resistance on system impedance at high frequencies. . . . . . . . 77

4.20 Floor- and ceiling-mounted transducer measurements. . . . . . . . . 78

5.1 Cavity volume on propeller blades at moderate rotation speed. . . . . . . 82

5.2 Cavitation compliance and mass flow gain factor estimates at moderate propeller rotation speed. . . . . . . . . . . . . . 83 
5.3 Cavitation compliance and mass flow gain factor estimates at high propeller

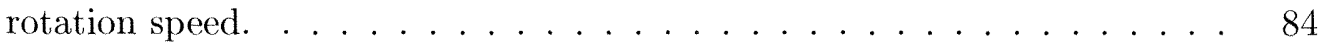

6.1 Schematic of system including only cavitation dynamics. . . . . . . . . 90

6.2 Schematic of local impedance. . . . . . . . . . . . . . . . . . . . 92

6.3 Schematic of facility and cavitation dynamics. . . . . . . . . . . . . . 94

6.4 Real part of system impedance for combined dynamics model. . . . . . . . 95

6.5 Schematic of simplified facility and cavitation dynamics. . . . . . . . . 97

6.6 Real part of system impedance for various cavitation compliances. . . . . 99

6.7 Effect of resistance on real part of system impedance. . . . . . . . . . . 101

6.8 Effect of unbalanced upstream and downstream resistances. . . . . . . . 103

6.9 Magnitude of system impedance for combined dynamics model. . . . . . . 104 


\section{List of Tables}

4.1 Measurements of the experimental facility. . . . . . . . . . . . . . 53

4.2 Numerical estimates of the lumped parameters. . . . . . . . . . . . 54

4.3 Revised estimates of the lumped parameters. . . . . . . . . . . . 72 


\section{Nomenclature}

The following symbols denote the various quantities encountered throughout the experiments and models.

\begin{tabular}{ll}
$c$ & chord length \\
$f$ & fluid friction factor \\
$f$ & frequency \\
$g$ & acceleration of gravity \\
$k$ & polytropic constant \\
$k$ & reduced frequency \\
$l$ & length \\
$m$ & mass flow rate \\
$n$ & frequency of propeller rotation \\
$p$ & pressure \\
$s$ & surface coordinate \\
$t$ & time \\
$u$ & flow velocity \\
$x$ & position \\
$A$ & cross-sectional area \\
$C$ & compliance \\
$D$ & diameter \\
$E$ & bulk modulus \\
$E$ & energy \\
$J$ & advance ratio \\
$K$ & cavitation compliance \\
$L$ & inertance \\
\hline & \\
\hline
\end{tabular}




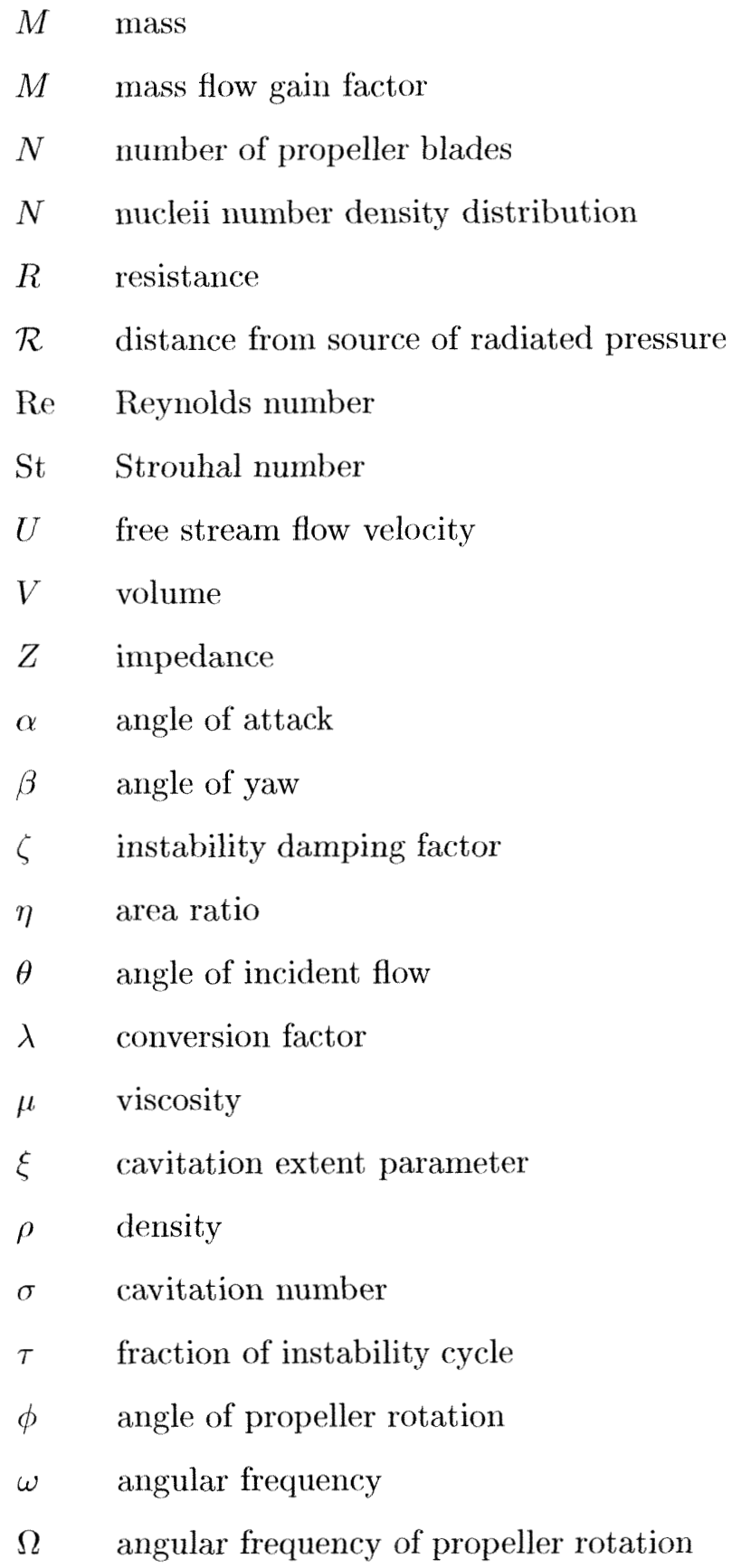


The following subscripts indicate under what conditions or at what location the above quantities are applicable.

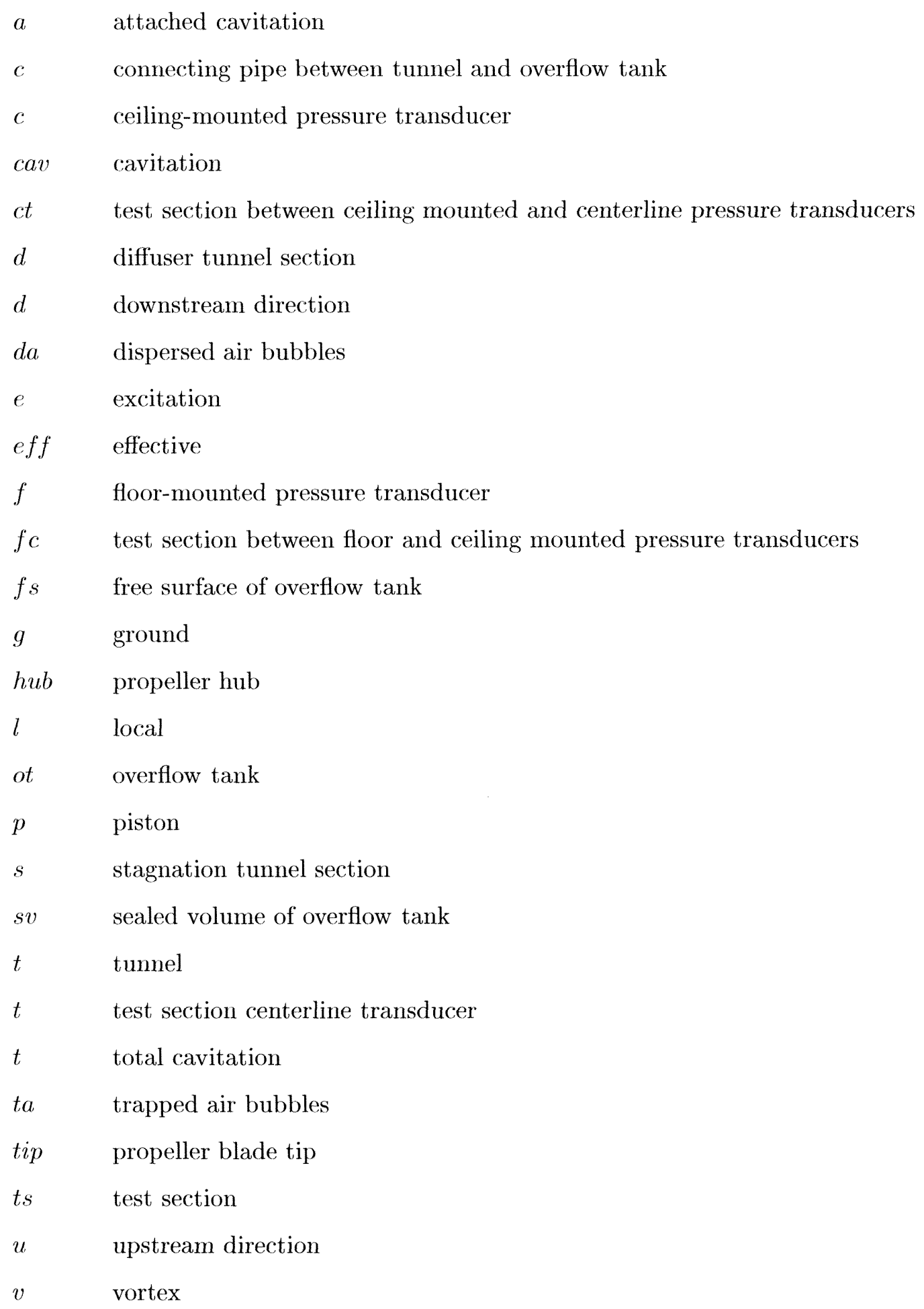




$\begin{array}{ll}w & \text { propeller wash } \\ x & \text { propeller cross-section } \\ L & \text { liquid } \\ R & \text { cavity boundary } \\ V & \text { vapor } \\ \infty & \text { far field }\end{array}$

The following superscripts indicate the nature of the above quantities.

$\begin{array}{ll}* & \text { dimensional } \\ T & \text { total } \\ S & \text { static }\end{array}$

In addition, the following mathematical notation is used.

$\bar{q} \quad$ steady or unperturbed value of quantity $q$

$\tilde{q} \quad$ complex fluctuating quantity $q$

$\bar{q} \quad$ conjugate of complex quantity $q$

$q_{o} \quad$ initial or original value of quantity $q$

$q_{o} \quad$ value of quantity $q$ at resonance or a transfer function pole

$q_{z} \quad$ value of quantity $q$ at a transfer function zero

$\Delta q \quad$ change in quantity $q$

$\operatorname{Arg}[q]$ phase angle of complex quantity $q$

$\operatorname{Re}[q] \quad$ real part of complex quantity $q$ 


\section{Chapter 1}

\section{Introduction}

In simplest terms, cavitation refers to the change of a fluid from a liquid phase to a gaseous phase in response to a reduction in local pressure below the vapor pressure of the fluid. Under some circumstances, the presence of cavitation can enable or enhance the operation

of a fluid mechanical device or process. Under the great majority of circumstances, though, cavitation degrades the performance of the device or process. Accordingly, cavitation has received a great deal of attention from the fluid mechanics research community.

\subsection{Adverse Effects of Cavitation}

The adverse effects of cavitation are numerous and varied, but are most conveniently categorized into the following four classes of phenomena.

- Noise. The vapor bubbles and cavities formed during the cavitation process are typically convected into regions of higher pressure within the flow, where through a very energetic collapse process, they return to the liquid phase. Some of this energy is emitted in the form of acoustic energy. The resulting noise may be a mere annoyance, or wholly unacceptable, as in the case of a naval craft seeking silent operation.

- Mechanical damage. The large energies associated with the collapse of the vapor bubbles and cavities can generate tremendously large local pressures that lead to rapid erosion of nearby solid surfaces. This can ultimately lead to catastrophic 
failure of the device.

- Decreased efficiency. The presence of vapor cavities, particularly on the suction surfaces of hydrofoils, pumps, and propellers, can substantially alter the nature of the flow over the surface. The end result of this change is often a dramatic decrease in the performance, such as lift or thrust, and efficiency of the device.

- Undesirable dynamics. The dynamics associated with the growth and collapse of vapor bubbles and cavities can lead to unsteady or unstable behavior of a device that does not occur in the absence of cavitation. This behavior can present very serious consequences in the system surrounding the device undergoing the resulting fluctuations in performance.

\subsection{Unsteady Cavitation Studies}

\subsubsection{Cloud Cavitation}

Experimental experience and theoretical analyses have revealed that the adverse effects of cavitation are often augmented under unsteady flow conditions. One particularly well documented phenomenon associated with unsteady flow is the production of coherent clouds of many vapor bubbles, termed cloud cavitation. Many investigators, including Knapp (1955), Bark and van Berlekom (1978), and Kubota, Kato, Yamaguchi, and Maeda (1989), have observed that the noise and mechanical damage resulting from the structurally complex collapse of cloud formations exceeds that implied by the aggregate contributions of the individual vapor bubbles. Analysis and simulation (van Wijngaarden 1964; d'Agostino and Brennen 1983; d'Agostino and Brennen 1989; Reisman, Wang, and Brennen 1998), have offered insight into the mechanisms responsible for this increase in noise and damage.

\subsubsection{Hydrofoils}

One very well studied source of cloud cavitation is the flow over a two-dimensional hydrofoil, in particular the phenomenon referred to as the partial cavity instability. Wade and Acosta (1966) first observed that the length of the cavity, $l$, on a two-dimensional 
hydrofoil of chord $c$ was stable when it was less than approximately $\frac{c}{2}$ or greater than $\frac{3 c}{2}$. At intermediate cavity lengths, the flow became unstable, and the cavity length fluctuated violently between these two limits of stability. Often, a cloud of cavitation bubbles was shed from the hydrofoil during each fluctuation cycle. Similar observations were made by Franc and Michel (1988), Le, Franc, and Michel (1989), and de Lange, de Bruin, and van Wijngaarden (1994).

The behavior of hydrofoils subject to forced oscillation in pitch about a spanwise axis has also been the subject of much research. Many investigators (Shen and Peterson 1978; Franc and Michel 1988; Hart, Brennen, and Acosta 1990; Soyama, Kato, and Oba 1992; McKenney and Brennen 1994; Reisman, Wang, and Brennen 1998) have noted that the periodic nature of the pressures on the suction surface of the oscillating hydrofoil can lead to even more dramatic production of cloud cavitation.

Until very recently, the great majority of this work focused on the behavior of twodimensional, unswept hydrofoils. However, the recent observations of attached cavities on hydrofoils with sweep by Jessup (1997) and Laberteaux and Ceccio (1998) have identified some important differences between the cavitation on three-dimensional swept hydrofoils and unswept two-dimensional hydrofoils. Most notably, the re-entrant flow on threedimensional hydrofoils was redirected normal to the line of cavity closure, rather than upstream, as is typical of two-dimensional hydrofoils. Also, the large regions of closed, attached cavitation on three-dimensional hydrofoils were generally steadier than the corresponding cavities on two-dimensional hydrofoils.

\subsubsection{Pumps}

Many of these investigations of the unsteady cavitation on hydrofoils suggest that the phenomena observed within the laboratory will also occur on a hydrofoil operating in more open conditions. Relatively few investigators (Shen and Peterson 1978; Kjeldsen, Vennatro, Arndt, and Keller 1999) have attempted to account for possible interactions between the dynamics of the unsteady cavitation and the dynamics of the surrounding experimental facility.

This is not the case for cavitating pumps, where for some time the dynamics of the 
pump cavitation and the response of the surrounding facility have been known to interact with very dramatic consequences. An example is the catastrophic POGO instability observed in liquid propellant rockets. A great deal of research has therefore been focused on quantifying the cavitation dynamics involved in oscillations of this nature. A relatively consistent approach has been adopted, with efforts aimed at developing a transfer matrix characterizing the relationship between the fluctuating pressure and mass flow rate at the pump inlet and discharge. This transfer matrix thus represents the dynamic behavior of the pump, including the cavitation.

In determining the elements of this transfer matrix, two important parameters have been identified. The cavitation compliance accounts for the effective compressibility of the cavitating flow between the inlet and outlet (Brennen and Acosta 1973) and was shown to play an important role in several observed hydraulic system instabilities. The mass flow gain factor, representing the response of the cavitation to variations in the inlet mass flow rate, was identified by Brennen and Acosta (1976). It was later demonstrated that this parameter, for which a typically positive value implies an increase in cavitation volume with decreasing inlet mass flow rate, is a key factor in sustaining instabilities such as cavitation surge (Brennen 1978; Tsujimoto, Kamijo, and Yoshida 1993).

The effects of the mass flow gain factor on the pump dynamics and the surrounding hydraulic system suggested by these analyses were confirmed experimentally (Braisted and Brennen 1978; Braisted and Brennen 1980) by observing the auto-oscillation of cavitating inducers. The first attempts to experimentally measure the mass flow gain factor as well as the cavitation compliance and the other components of the transfer matrix were performed by $\mathrm{Ng}$ and Brennen (1978). More precise measurements were later obtained by Brennen, Meissner, Lo, and Hoffman (1982). More recently, the frequency dependence of the mass flow gain factor has been explored theoretically by Otsuka, Tsujimoto, Kamijo, and Furuya (1996).

\subsubsection{Propellers}

The majority of investigations exploring the unsteady cavitating behavior of propellers has focused on the consequences of the varying inflow encountered by a propeller blade as it 
rotates through the irregular wake behind a ship hull. Huse (1972) was the first to explore the resulting variations in cavity volume and their effect on the pressures generated by the cavitation. The amplitude of the pressure fluctuations and the induced vibrations of the ship hull were found to be orders of magnitude greater than for the case of steady cavitation. A summary of the large body of work focused on the propeller-hull vibration problem is offered in Weitendorf (1989). A discussion of the various unsteady cavitation structures that can result from the irregular wake is presented by Bark (1986). McKenney (1995) explored the unsteady flow field encountered by a yawed propeller and its effect on tip vortex cavitation.

\subsection{Current Work}

Few if any cavitating propeller investigations have adopted an approach similar to the cavitating pump analyses and explored the possible interaction between unsteady propeller cavitation and the dynamics of the surrounding environment. The goal of this work is to utilize concepts garnered from general unsteady cavitation research and extend several specific concepts from investigations of cavitating pump instabilities to describe and explain a previously unobserved cavitation surge instability on a propeller.

The instability is characterized by a periodic fluctuation in cavitation extent on the propeller blades and in the tip vortices. The resulting pressure fluctuations are dramatic, and propagate throughout the surrounding experimental facility. It is hoped that a better understanding of the instability will help mitigate the potential increase in propeller damage and hull vibration should the instability occur in a ship wake environment.

The details of the experimental facilities and techniques employed throughout the work are presented in Chapter 2. The steady cavitating behavior of the propeller is described in the initial portion of Chapter 3, with observations made regarding the extent and nature of the cavitation under varied experimental conditions. The remainder of Chapter 3 describes the observed cavitating propeller instability, including the nature and extent of the cavitation throughout the instability cycle, the fluctuating pressures generated by the instability, the onset of the instability, and the variation of the instability frequency. 
To investigate the possible effects of the surrounding hydraulic system, a model for the facility dynamics is developed in Chapter 4 . Chapter 5 , drawing on some of the results obtained in Chapter 3, more precisely quantifies the dynamics of the propeller cavitation. These separate dynamics are then considered together in Chapter 6 to offer insight into the origin and behavior of the instability. Finally, conclusions drawn from the research and recommendations for future topics of investigation are presented in Chapter 7. 


\section{Chapter 2}

\section{Experimental Facilities and Methods}

Summarized in this chapter are the experimental facilities and methods used throughout the investigation. All of the experiments were conducted in the Keck Engineering Laboratory on the Caltech campus. A combination of existing and original experimental equipment was employed.

\subsection{Low Turbulence Water Tunnel Facility}

The experiments investigating the cavitating behavior of the model propeller were conducted in the Low Turbulence Water Tunnel (LTWT) facility. Originally designed to study the effect of particulates on turbulent flows, the later addition of a vacuum system allowed for the study of cavitation phenomena. A complete description of the facility can be found in Gates (1977).

An overview of the features of the facility is given in Figure 2.1. A $22.5 \mathrm{~kW}$ DC motor driving a mixed flow pump is used to vary the flow rate in the tunnel. At peak output from the pump, the $16: 1$ contraction ratio produces a flow velocity of approximately $10 \mathrm{~ms}^{-1}$ through the test section, which has a length of $2.5 \mathrm{~m}$ and a $0.3 \mathrm{~m}$ square cross-section. A vacuum pump applied to the interior of an overflow tank containing the free surface of the facility is used to adjust the static pressure throughout the facility. In this manner, the test section static pressure can be varied from 20 to $112 \mathrm{kPa}$.

The test section static pressure and the flow velocity are calculated by a Zenith Intel 8086 based computer which monitors the output of pressure transducers connected to 


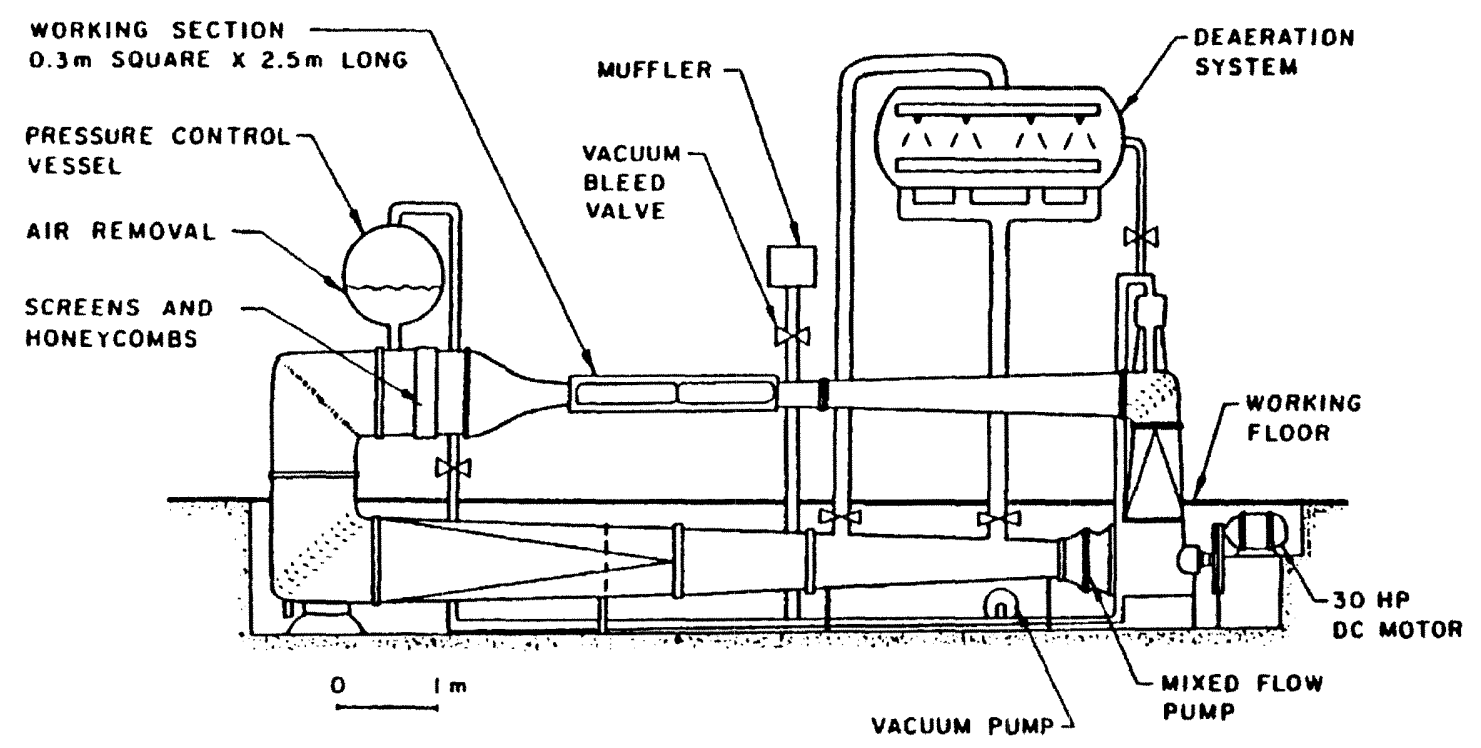

Figure 2.1: Overview of Low Turbulence Water Tunnel facility.

taps both upstream and downstream of the contraction. During the cavitating propeller experiments, the pressure transducer signals were corrupted by noise from the inverter driving the propeller motor. In this case, the pressure measurements were taken from a manometer system connected to the same set of pressure taps.

The facility is also equipped with a deaeration system to control the air content within the tunnel. This allows for the total air content, incorporating both dissolved air and free air bubbles within the facility, to be varied from approximately $14 \mathrm{ppm}$, upon filling the facility, to as low as $3 \mathrm{ppm}$. The total air content is determined using a Van Slyke magnematic blood gas apparatus. Finally, corrosion is controlled through the addition of sodium chromate to the water within the facility. The effectiveness of this additive is enhanced with the further addition of potassium hydroxide. 

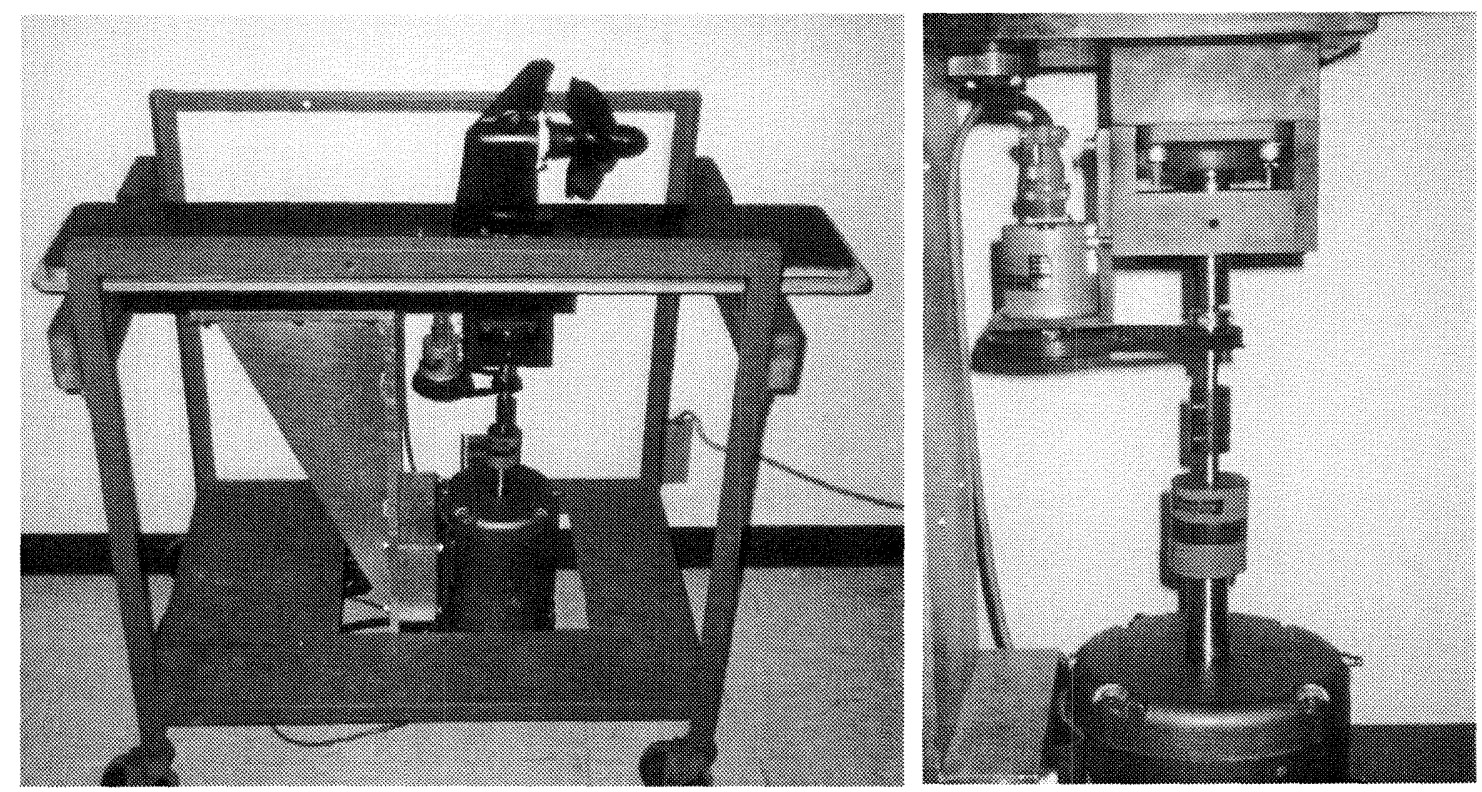

Figure 2.2: Propeller drive experimental setup. Shown at left is the propeller and propeller drive assembly with the propeller mounted upstream of the gear case, outside of the tunnel test section on an assembly cart. At right is a detailed view of the motor, drive shaft, bearing and seal assembly, and optical shaft encoder.

\subsection{Propeller Experiments}

\subsubsection{Propeller and Propeller Drive}

Photographs of the propeller drive assembly used in the cavitating propeller experiments are shown in Figure 2.2. Originally developed and described in detail by McKenney (1995), this assembly is composed of a $3.75 \mathrm{~kW}$ motor, drive shaft, bearing and seal assembly, right angle gear case, and propeller shaft. The motor, drive shaft, and bearing and seal assembly are supported by a right angle bracket that is attached to the underside of the test section bottom panel. The right angle gear case and propeller shaft are mounted flush to the opposing side of this panel, placing them within the tunnel test section.

The gear case and propeller shaft subassembly can be pivoted about the gear case base, allowing the propeller to be operated at various yaw angles with respect to the incoming flow. A full half turn of the gear case in this manner enables the propeller to be operated either upstream or downstream of the support strut. During such a change in configuration, the propeller was remounted on the propeller shaft to restore proper 

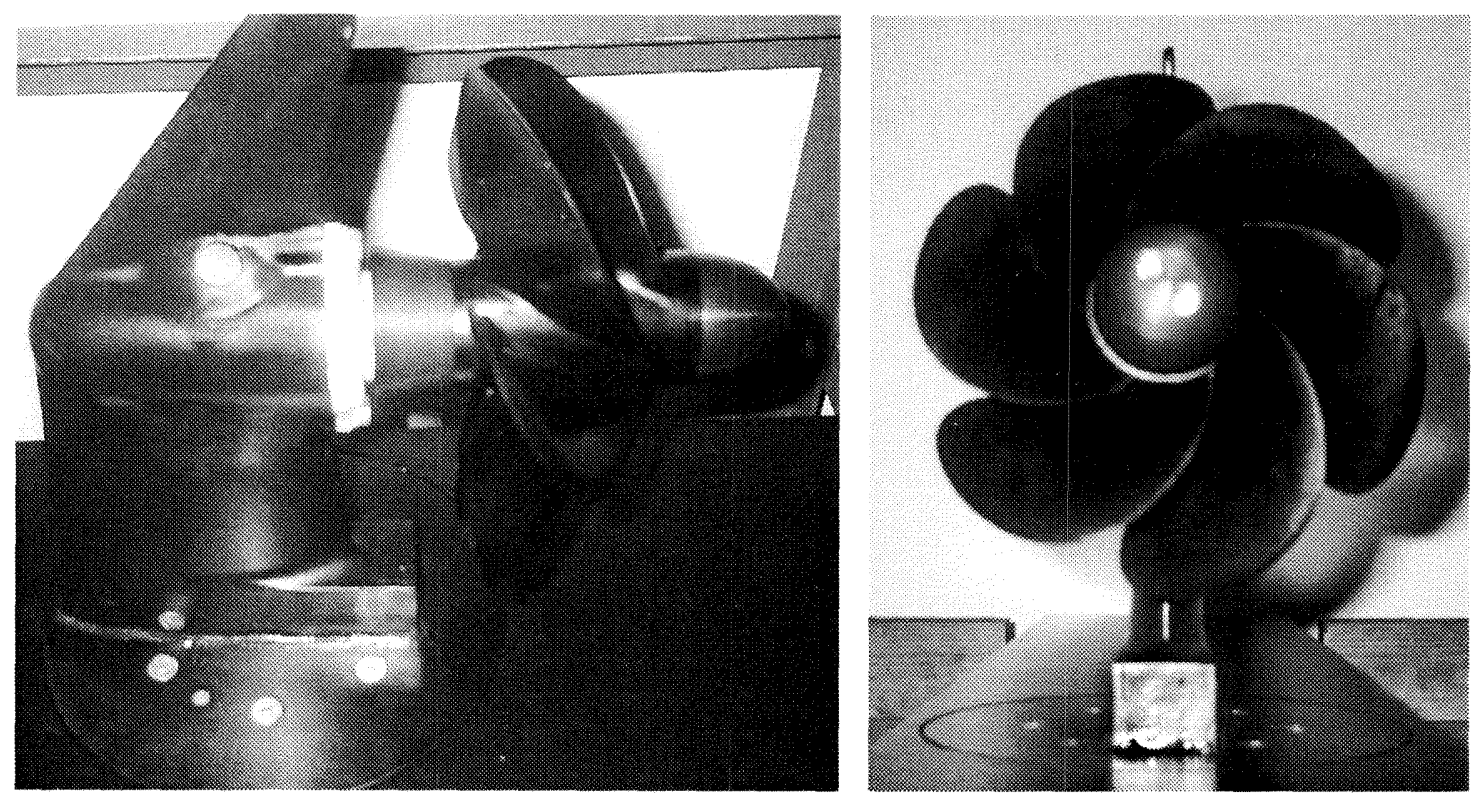

Figure 2.3: Propeller, propeller mount, and right angle gear case.

orientation relative to the incoming flow. More detailed photographs of the propeller and gear case assembly are provided in Figure 2.3.

The propeller utilized in the current experiments is different than that described in the work of McKenney (1995). On loan from the David Taylor Model Basin, Navy Model Propeller 5236 is a 1:6.7 scale model of a patrol boat design. The six bladed model has a maximum blade chord length of $88.0 \mathrm{~mm}$, a diameter of $190.5 \mathrm{~mm}$, a hub diameter of $47.6 \mathrm{~mm}$, and a bore diameter of $12.7 \mathrm{~mm}$. An effective radius, representing the average radius in an areal sense, can be defined as

$$
R_{e f f}=\frac{2}{3} \frac{R_{t i p}{ }^{3}-R_{h u b}{ }^{3}}{R_{t i p}{ }^{2}-R_{h u b}{ }^{2}}
$$

and evaluated as $R_{e f f}=66.7 \mathrm{~mm}$. The chord length at this effective radius, $c_{e f f}=$ $87.8 \mathrm{~mm}$, is very nearly the maximum chord length.

The bore diameter of the new propeller is larger than the diameter of the drive assembly propeller shaft. A sleeve for the existing shaft was machined to accommodate this discrepancy in diameters. A diametric pin assures that the sleeve rotates with the underlying shaft. Threads at the end of the sleeve nearest the gear case allow for attach- 
ment of a tapered skirt to smooth the flow between the propeller hub diameter and the diameter of the gear case bearing and seal assembly. Finally, a nose cone is attached with axial screws to the opposite end of the sleeve, clamping the propeller hub between the nose cone and skirt. The semi-ellipsoidal nose cone is shaped to yield a smooth inflow to the propeller when operating upstream of the gear case and minimize hub vortex cavitation when operating downstream of the gear case. The skirt and nose cone are readily distinguished in the photographs of Figure 2.3.

The existing motor in the McKenney propeller drive was capable of operating speeds up to $3450 \mathrm{rpm}$, providing propeller rotation speeds up to $1550 \mathrm{rpm}$ after reduction by the gear case. To explore higher propeller speeds, a precision balanced, fan cooled Baldor motor was installed to permit motor speeds of $5000 \mathrm{rpm}$ and propeller speeds of $2250 \mathrm{rpm}$. However, depending on the tunnel flow velocity, propeller speeds in excess of $1900 \mathrm{rpm}$ typically maximize the power handling capabilities of the Sabina type 9558 inverter used to drive the motor.

Visible in Figure 2.2 is a BEI Motion Systems optical shaft encoder attached to the drive shaft of the propeller drive assembly. The 1440 pulses per revolution output form this encoder are monitored by a timing box with circuitry that, adjusting for the reduction provided by the gear case, determines the propeller rotation speed. The timing box can also be used to provide triggers for other equipment such as strobe lights and data acquisition software at a selected angle of propeller rotation.

\subsubsection{Pressure Measurements}

Pressure transducers were installed at various locations within the tunnel test section to record the pressures generated by the cavitating propeller. PCB Piezotronics model HS113A21 quartz pressure transducers, with a $100 \mathrm{kHz}$ bandwidth, were flush-mounted in both the floor and ceiling of the tunnel test section. As shown in Figure 2.4, the floormounted transducer was placed almost directly underneath the propeller when configured downstream of the gear case, while the ceiling-mounted transducer was placed approximately $0.40 \mathrm{~m}$ upstream. A more detailed discussion of the installation and operation of these transducers is provided by Reisman (1997). 


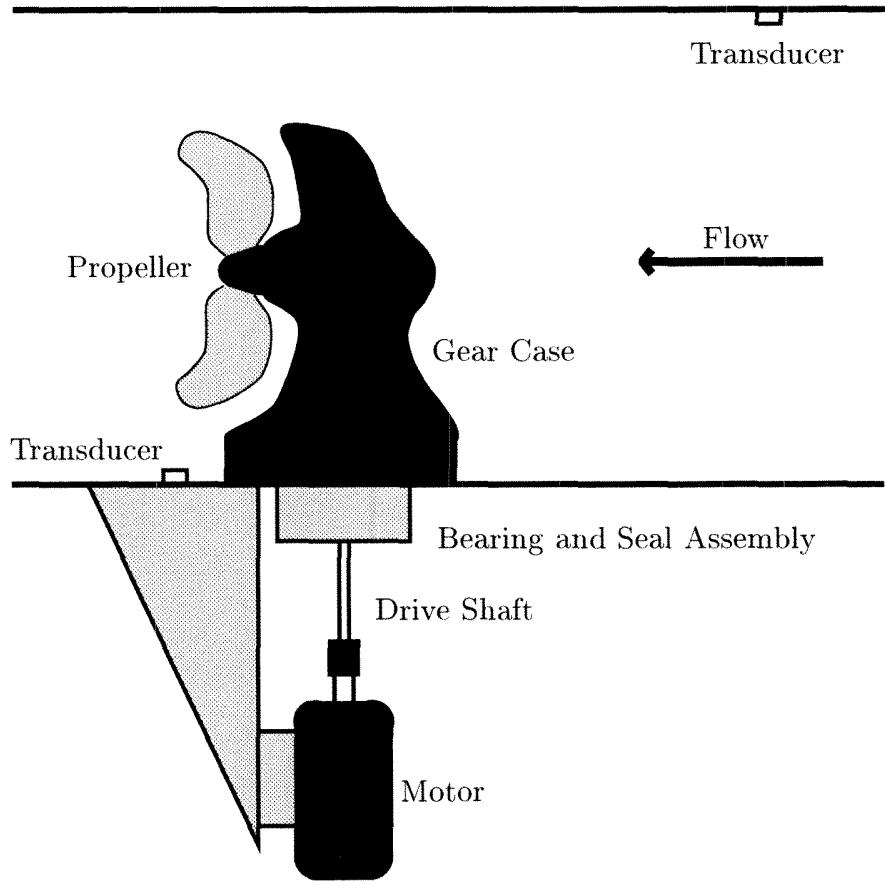

Figure 2.4: Schematic of propeller drive installation and pressure transducer locations within tunnel test section. The propeller is shown here mounted downstream of the gear case. The pressure transducers flush-mounted to the floor and ceiling of the test section are also shown.

\subsubsection{Visual Observations}

\section{Direct Observations}

In many cases, the most efficient method of obtaining experimental data was through direct visual observation of the propeller. This was accomplished through the use of one or more strobe lights, triggered by a pulse from the timing box described in Section 2.2.1. Strobe lights from several manufacturers were used, including an EG\&G Optoelectronics 549 Microflash and a General Radio Strobotac 1531-A. The best results were obtained with a Chadwick Helmuth system consisting of a 8490-5 Strobex slave power supply and a $270 \mathrm{D}$ light head. This system is capable of responding to triggering rates as high as $500 \mathrm{~Hz}$, and produces flashes of approximately $5 \mu \mathrm{s}$ duration.

Typically, the strobes were triggered once per revolution, producing a phase-averaged view of the propeller at the desired rotation position. During relatively steady operation of the cavitating propeller, this allowed for reliable visualization of the cavitation. 


\section{Still Photographs}

In those cases where a phase-averaged view of the propeller was not adequate and in cases where recording the propeller cavitation was desired, still photographs were taken. This was accomplished by opening the camera shutter in a completely darkened laboratory, producing a single strobe flash, then closing the camera shutter. In this manner the shutter speed of the camera was effectively reduced to the duration of the strobe flash.

Proper adjustment of the camera aperture was therefore essential, but could only be determined through a trial and error process. Uncertainties and inconsistencies in the processing of color print film posed great difficulties during this iterative process. Despite attempts with various different color print films, including Kodacolor Gold 400, Fujicolor Super G Plus 400, and Fujicolor Super HG 1600, truly satisfactory results could not be achieved. The greater certainty and repeatability of slide film processing allowed for much better imaging. The majority of slide images were obtained using Kodak 400 Elite II color slide film.

\section{High Speed Movies}

High speed motion pictures of the cavitating propeller were also obtained. The films were acquired with a LOCAM II $16 \mathrm{~mm}$ high speed motion picture camera manufactured by Redlake Corporation. This camera offers framing rates up to $500 \mathrm{fps}$ and shutter

speeds as fast as $\frac{1}{36000} s$. Several early experiments were filmed using Kodak Ektachrome 7250 color reversal film, but better results were achieved with Kodak Vision 500T color negative film.

Due to the extremely fast shutter speeds required to freeze the propeller motion effectively, providing sufficient lighting for the camera was a challenge. Various $500 \mathrm{~W}$ halogen lamps were used, but superior lighting was obtained using several Lowel Pro lights. Although these lights are rated at only $250 \mathrm{~W}$, their lamps contain higher temperature filaments with an emission spectrum more closely matched to the sensitivity spectrum of the film, and their reflectors yield improved directionality of the emitted light.

Improved image quality was also obtained after extensive experimentation revealed that a bright red paint applied to the propeller reflected copious amounts of light from the 
propeller blades but still allowed for sharp distinction of both clear and bubbly cavitation. The choice of backdrop behind the tunnel test section also played a role in determining the quality of the films, with black felt providing the best results.

\section{High Speed Video}

The high speed movie camera described above is very tedious to load and operate, and processing of the film, particularly color negative film, is very costly. As such, the great majority of motion capture was performed with a high speed digital video camera. This system, also manufactured by Redlake, offers an equal framing rate of $500 \mathrm{fps}$ but a slightly slower shutter speed of $\frac{1}{1.0000} s$. The lighting used in conjunction with the digital camera was the same as that used for the film camera. Video acquired by the system was archived through transfer to VHS video tape.

\subsection{Oscillating Piston Experiments}

\subsubsection{Oscillating Piston Mechanism}

Experiments exploring the response of the facility to a volumetric excitation were conducted using the setup shown schematically in Figure 2.5. The output from an oscillating piston was directed into the tunnel test section through a short run of essentially rigid pipe. The mechanism used to oscillate the piston was adapted from the oscillating hydrofoil apparatus originally developed by Hart (1993). A $750 \mathrm{~W}$ motor was used to rotate a shaft to which a linkage leading to the piston was eccentrically coupled. To achieve very low oscillation frequencies, the motor was connected to a worm gear box with a 15:2 reduction, which was then coupled to the eccentric shaft. This ensured smooth operation of the piston even at extremely low frequencies. The motor, gear box, bearing blocks for the shaft, and piston were all rigidly mounted to a heavy aluminum base plate.

Experiments were conducted with the base plate in several locations, but the best results were achieved with the plate on top of the test section, as this allowed for the shortest length of pipe between the piston and injection point. This minimized the pressure fluctuations associated with the acceleration of the fluid within the connecting pipe, 


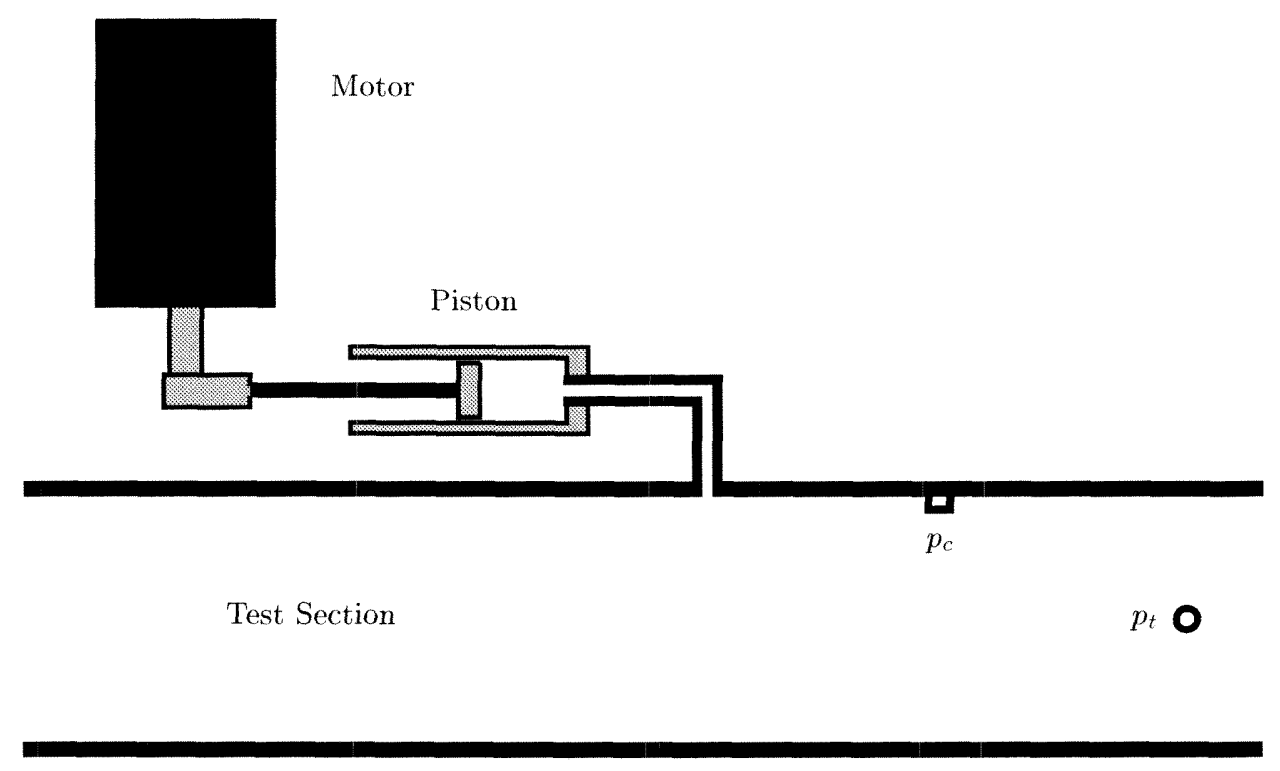

Figure 2.5: Oscillating piston experimental setup.

and therefore also minimized the likelihood of cavitation on the piston face during suction strokes. In this configuration, the aluminum base plate was directly and rigidly attached to the tunnel test section frame.

The range of oscillation frequencies explored was $0-20 \mathrm{~Hz}$, controlled by direct variation of the motor speed. Frequencies above $f=20 \mathrm{~Hz}$ resulted in excessive vibration of the motor and piston assembly. The eccentricity of the connection between the piston and motor was variable, allowing a range of oscillating piston amplitudes of 2.0 to $15.0 \mathrm{~mm}$ to be investigated. To ensure that the motion of the piston remained as nearly sinusoidal as possible, the pressure within the facility was adjusted to balance the pressures on either side of the oscillating piston.

The piston, manufactured by Bimba Manufacturing Company, was originally designed for use as a pneumatic cylinder, but due to its stainless steel and aluminum construction and well designed seals, it performed adequately as a hydraulic piston. Extensive wear, though, required that the pistons be periodically replaced. The piston bore diameter and bore length are $38 \mathrm{~mm}$ and $70 \mathrm{~mm}$, respectively. The original outlet from the piston was plugged and a new opening was drilled and tapped to allow for direct connection of the $\frac{3}{4}$ in pipe leading to the test section. 


\subsubsection{Pressure Measurements}

The fluctuating pressures within the facility resulting from the piston motion were measured with two pressure transducers, as shown in Figure 2.5. The flush-mounted transducer in the test section ceiling described in Section 2.2.2 recorded the fluctuating static pressure $0.51 \mathrm{~m}$ upstream from the piston injection point. In addition, a Setra Systems model $239 \mathrm{E}$ transducer recorded the fluctuating static pressure at the tunnel mid-line $0.41 \mathrm{~m}$ further upstream. The $10 \mathrm{~ms}$ response time of this transducer was sufficient to capture the excitation frequency of the oscillating piston. Both the frequency and the amplitude of the pressure oscillations were determined through cross-correlation of the measured response with complex fluctuating responses of various frequencies. Finally, by monitoring the 1024 pulses per revolution output of an optical shaft encoder mounted on the motor driving the oscillating piston, the phase difference between the piston motion and pressure oscillations could also be determined.

\subsection{Data Acquisition and Processing}

During the earliest experiments, data acquisition of the pressure transducer and shaft encoder signals was performed by an R.C. Electronics ISC-16 digital data acquisition card and accompanying software operating on an Intel 80486 based computer. The performance of this system is described in Reisman (1997). Following the failure of this system, a ComputerBoards CIO-DAS801 system was installed in an Intel Pentium based computer. This system is capable of acquiring up to eight channels of data at a sampling rate of $50 \mathrm{kHz}$.

In many cases, signal conditioning was performed in the laboratory at the time of the experiment. To prevent aliasing, low-pass filtering was performed prior to analog to digital conversion using a Krohn-Hite 3202R four channel filter. When needed, amplification was provided by amplifiers assembled by Caltech technicians. In addition, a Stanford Research Systems SR560 was used for amplifying and filtering a single channel. Any further conditioning needed and all post-experiment data analysis was conducted using routines written in MathWorks' MATLAB technical computing software. 


\section{Chapter 3}

\section{Cavitating Propeller Experiments}

Upon installation of the model propeller in the LTWT facility, experiments began with the propeller mounted upstream of the gear case. This avoided any complications associated with the flow irregularities present in the wake of the gear case. These early investigations revealed insight into the steady cavitating behavior of the propeller at a range of operating conditions.

The nature and focus of the work changed dramatically when the propeller was operated downstream of the gear case. Immediately apparent following this change in configuration was an unstable cavitating behavior of the propeller. Efforts were subsequently focused on better documenting and understanding this instability.

\subsection{Experimental Parameters}

As described in Chapter 2, the conditions within the test section of the facility were varied by adjusting the propeller yaw angle, propeller rotation speed, test section static pressure, and test section free stream velocity. Each of these factors influences the extent of cavitation present or the likelihood of cavitation inception if none is present.

Since cavitation occurs where local pressures below vapor pressure are attained in the flow, it is clear that lowering the ambient static pressure within the test section will increase the extent of cavitation present. Furthermore, the ability of the propeller to create the requisite pressure reduction is related to its rotation speed, with higher speeds leading to more dramatic spatial variation in local pressure. These two concepts are 

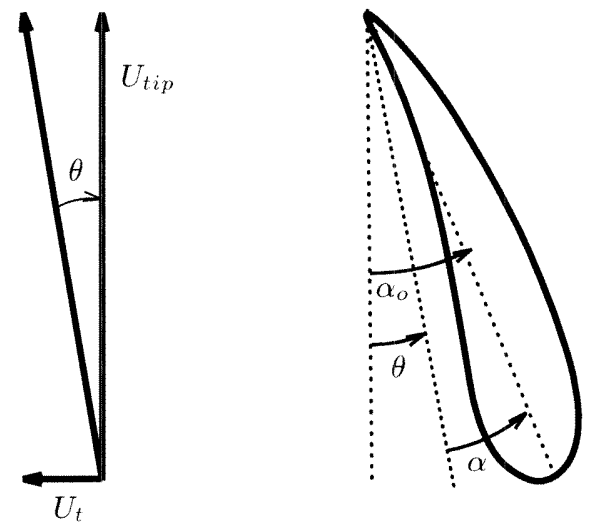

Test Section Flow

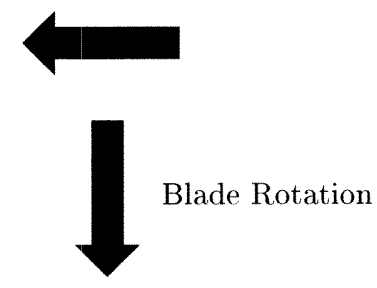

Figure 3.1: Effective angle of attack of propeller blade.

effectively summarized by the dimensionless cavitation number,

$$
\sigma=\frac{p_{t}-p_{V}}{\frac{1}{2} \rho U_{t i p}^{2}}
$$

where $p_{t}$ is the test section centerline static pressure, $p_{V}$ the vapor pressure of water, $\rho$ the density of water, and $U_{t i p}$ the tip speed of the propeller.

Also of great importance in determining the extent of cavitation present is the effective angle of attack of the individual propeller blades, as this determines the amount of pressure reduction that will occur on the suction surfaces. As shown in Figure 3.1, the tip velocity and the tunnel velocity, $U_{t}$, combine to yield an effective angle of attack, $\alpha$, below the geometric angle of attack, $\alpha_{o}$. More precisely,

$$
\begin{aligned}
& \alpha=\alpha_{o}-\arctan \left[\frac{U_{t}}{U_{t i p}}\right] \\
& \alpha=\alpha_{o}-\arctan \left[\frac{J}{\pi}\right],
\end{aligned}
$$

where the advance ratio has been introduced, defined as

$$
J=\frac{\pi U_{t}}{U_{t i p}}=\frac{U_{t}}{n D}
$$

Here $n$ is the rotation speed of the propeller, in cycles per second, and $D$ the propeller diameter. The advance ratio reflects the propulsive effort provided by the propeller, in 
that at a sufficiently high advance ratio, the propeller will no longer provide any thrust at all but will instead slip smoothly through the flow.

This analysis becomes more complex when the propeller is yawed relative to the test section flow. Then, the effective angle attack is not merely a function of the test section flow velocity and propeller rotation speed, but angular position as well (McKenney 1995), with

$$
\alpha=\alpha_{o}-\left[\frac{J \cos \beta}{\pi+J \sin \beta \cos \phi}\right],
$$

where $\beta$ is the yaw angle and $\phi$ is the angular position of the propeller blade relative to top dead center.

\subsection{Stable Cavitating Behavior}

\subsubsection{Propeller at Zero Yaw}

Experiments were run with the propeller mounted upstream of the gear case to investigate the behavior of the propeller under various parametric conditions. Operation in this configuration was typically characterized by steady cavitating behavior, and was therefore amenable to both the direct visual observation and photographic techniques described in Section 2.2.3.

Figure 3.2 shows a photograph of the propeller under moderate cavitating conditions. Visible in the photograph are many features characteristic of the cavitation typically observed on the model propeller. Most striking is the helical pattern of the cavitating tip vortices shed from the propeller tips, persisting for several propeller diameters downstream despite interference from the gear case. Closer inspection of the cavitation on the propeller blades reveals that the cavitation may be divided into two principal regions of clear, attached cavitation, and bubbly, detached cavitation. These features are shown schematically in Figure 3.3.

Also detailed in Figure 3.3 is a method of quantifying the extent of cavitation present on the propeller blades. The cavitation extent varies substantially with radial location, 


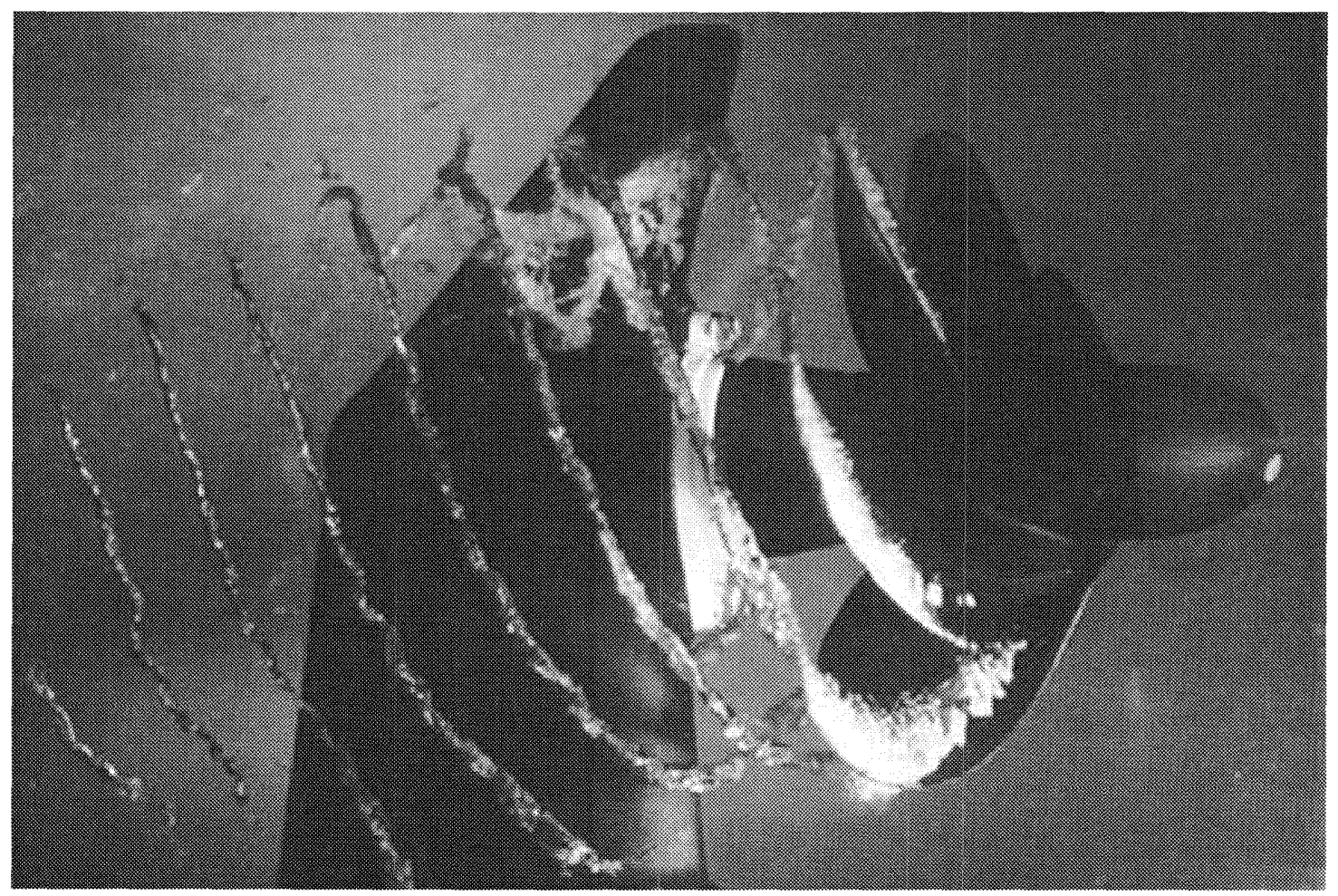

Figure 3.2: Photograph of stable propeller cavitation. Propeller cavitating upstream of the gear case with $\beta=0^{\circ}, n=28.3 \mathrm{~Hz}, J=0.68$, and $\sigma=0.19$.

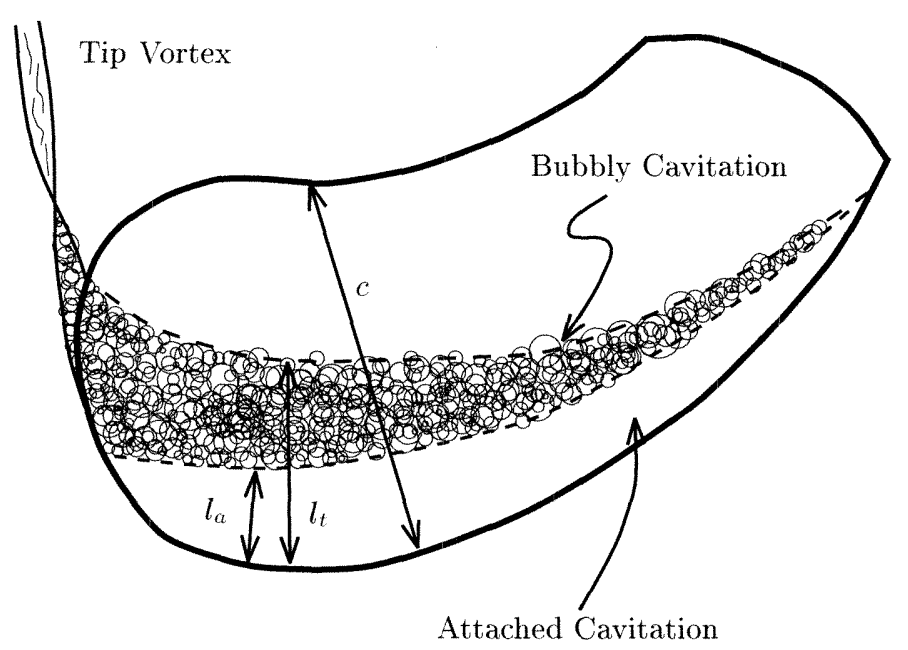

Figure 3.3: Schematic of cavitation features and cavitation extent. 
but is most easily characterized by a single length, $l_{t}$, the maximum cavitation extent measured locally perpendicular to the leading edge. A second measure defined in the same manner is the length, $l_{a}$, of the attached cavity. These lengths can be non-dimensionalized by $c$, the maximum chord length of the propeller.

Figure 3.4 shows the cavitation extents, $\frac{l_{t}}{c}$ and $\frac{l_{a}}{c}$, for a propeller at zero yaw rotating at $n=25.0 \mathrm{~Hz}$, at various cavitation numbers and advance ratios. Readily apparent is that more cavitation is present at lower advance ratios and cavitation numbers, consistent with the arguments of Section 3.1. This data was obtained by maintaining a constant test section flow velocity while varying the test section static pressure. Experiments were also conducted by varying the test section flow velocity at a constant test section static pressure, and similar results were obtained.

Figures 3.5 and 3.6 show data obtained at higher propeller rotation speeds. The flow conditions investigated are somewhat different for each of the rotation speeds. In particular, the range of cavitation numbers is lower for higher speeds as the increased propeller speed results in lower cavitation numbers across the range of attainable test section pressures. Also, because a considerable test section flow velocity was required to avoid overloading the driving inverter when operating the propeller at the highest rotation speed, the minimum advance ratio investigated at that rotation speed is higher than at the lower rotation speeds.

In general, the data at these higher propeller rotation speeds exhibits similar trends to those seen in Figure 3.4. Careful comparison of the three figures, though, reveals some important distinctions. Even at identical cavitation numbers and advance ratios, the extent of cavitation observed is substantially different at various propeller rotation speeds, with higher speeds resulting in more cavitation. This suggests that the cavitation number does not completely account for the effects of rotation speed as implied in Section 3.1.

At the lower rotation speeds, the attached cavitation fraction is more strongly a function of cavitation number than advance ratio. At the highest rotation speed, though, the attached cavitation fraction changes more noticeably with varying advance ratio. This is likely attributable to the fact that at the highest rotation speed, the attached cavitation composes a larger portion of the total cavitation extent and therefore follows more closely 

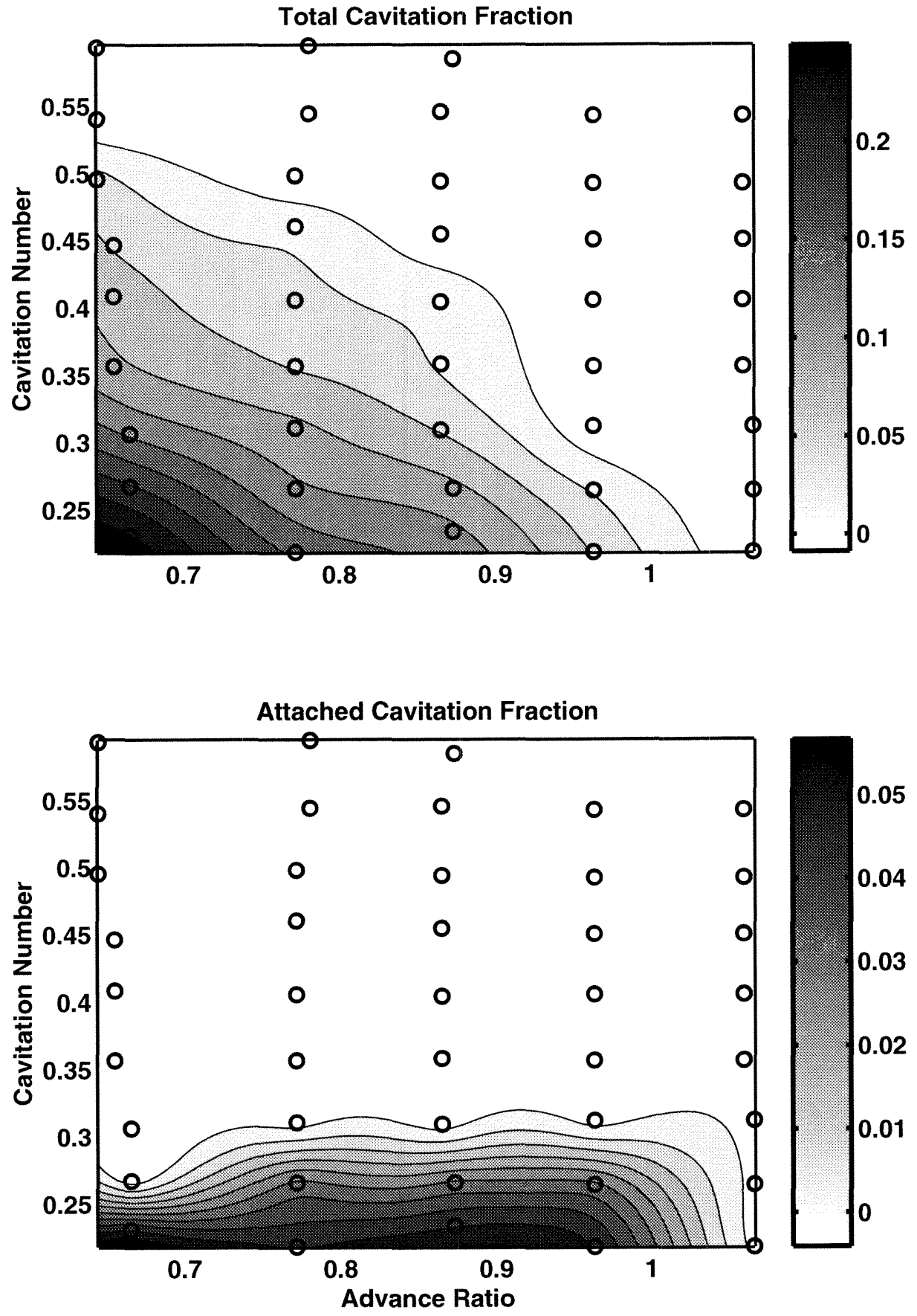

Figure 3.4: Cavitation extent at low rotation speed. Propeller operating at $\beta=0^{\circ}$, $n=25.0 \mathrm{~Hz}$. Upper plot shows the total cavitation fraction, $\frac{l_{t}}{c}$, lower plot the attached cavitation fraction, $\frac{l_{a}}{c}$. The contours are based on a fit to the experimental data points (o). 

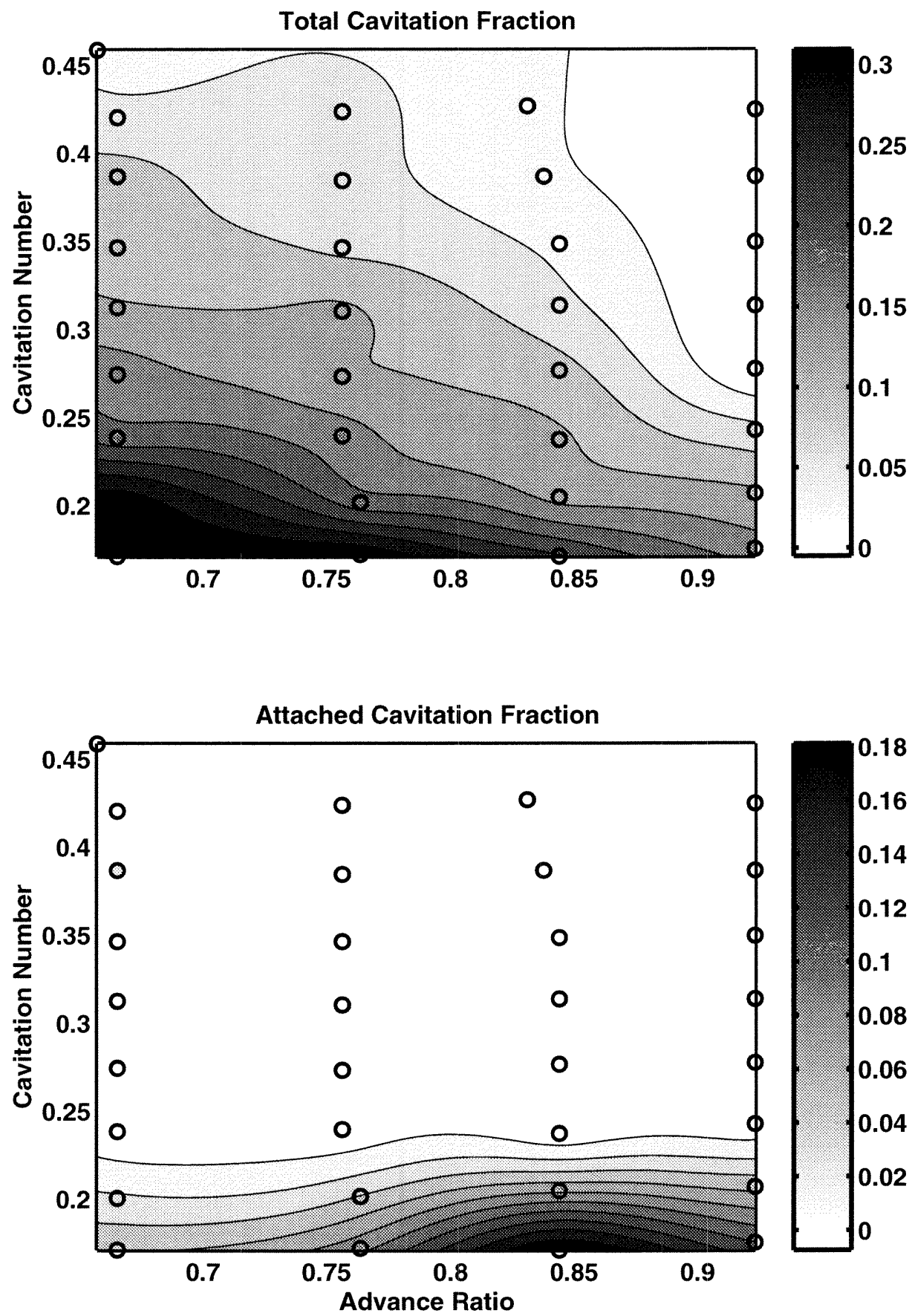

Figure 3.5: Cavitation extent at moderate rotation speed. Propeller operating at $\beta=0^{\circ}$, $n=28.3 \mathrm{~Hz}$. Upper plot shows the total cavitation fraction, $\frac{l_{t}}{c}$, lower plot the attached cavitation fraction, $\frac{l_{a}}{c}$. The contours are based on a fit to the experimental data points $(0)$. 

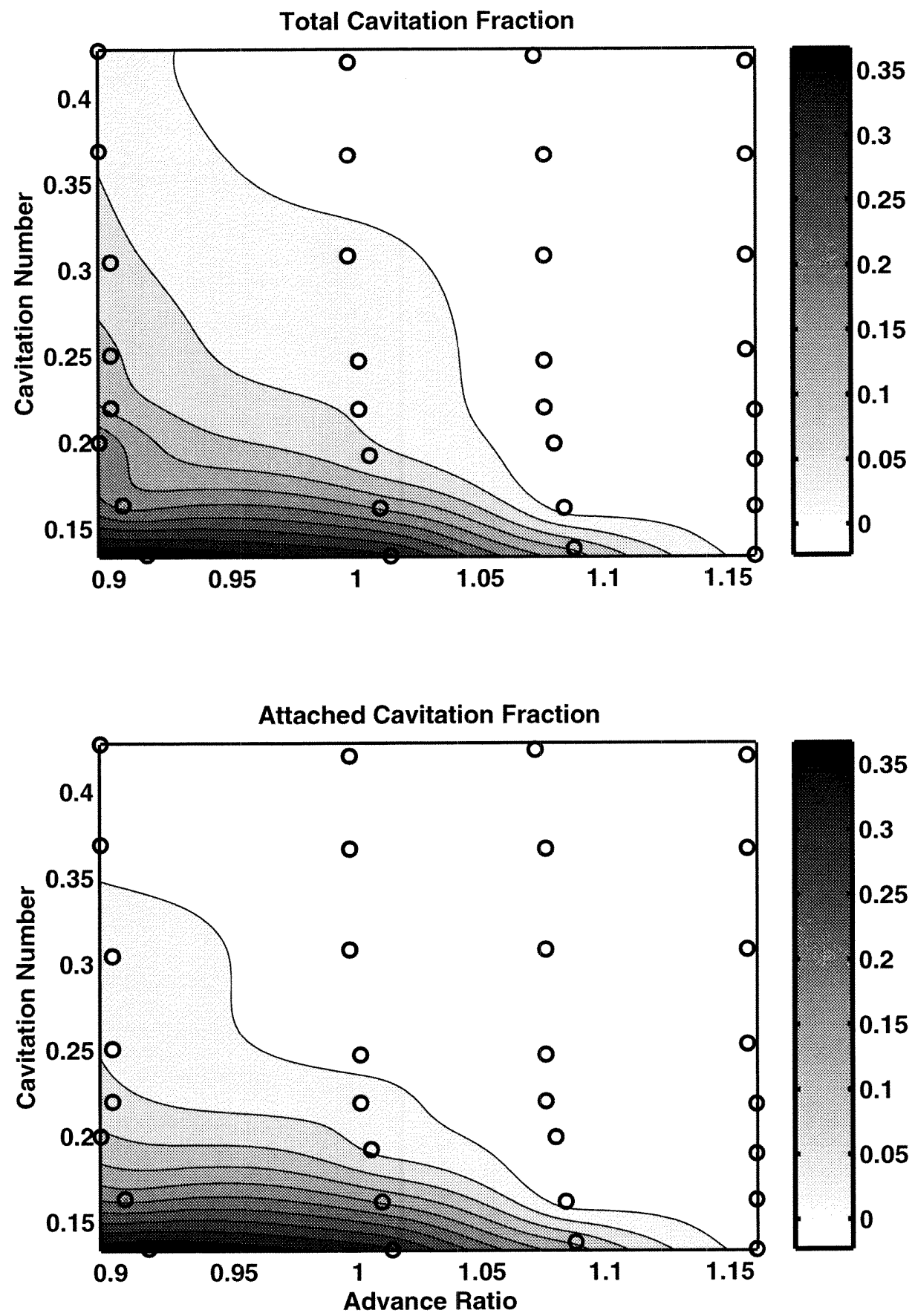

Figure 3.6: Cavitation extent at high rotation speed. Propeller operating at $\beta=0^{\circ}$, $n=31.7 \mathrm{~Hz}$. Upper plot shows the total cavitation fraction $\frac{l_{t}}{c}$, lower plot the attached cavitation fraction $\frac{l_{a}}{c}$. The contours are based on a fit to the experimental data points (0). 
the trend seen for the total cavitation fraction at all rotation speeds. This discrepancy also implies that the cavitation number and advance ratio do not completely encapsulate the experimental conditions, and therefore do not summarize the cavitating behavior of the propeller as effectively as one might hope.

Also noteworthy is that the data taken at $25.0 \mathrm{~Hz}$ and particularly $28.3 \mathrm{~Hz}$ shows a decrease in the extent of attached cavitation below an advance ratio of approximately $J=0.75$. This is in opposition to the overall trend of increasing cavitation with decreasing advance ratio. Unfortunately, due to the restriction in the minimum achievable advance ratio, this trend could not be explored at the highest propeller rotation speed.

\subsubsection{Propeller at Yaw}

The behavior of the propeller at yaw was also considered. In these experiments, the gear case and propeller assembly were rotated such that the upstream propeller axis was right of the incoming flow, corresponding to a negative value of the yaw angle defined in Equation 3.5. Observations were also made with the propeller placed at a positive yaw angle, with no noticeable difference in the results.

Shown in Figure 3.7 are the total cavitation extents measured at various angular positions on the propeller disc of rotation. For comparison, the effective angle of attack of Equation 3.5 is also shown. This calculation is based on a geometric angle of attack of $a_{o}=22^{\circ}$, an estimate of the geometric angle of attack at the effective propeller radius, $R_{e f f}=0.7 R_{t i p}$. This choice of $\alpha_{o}$ is somewhat arbitrary, as the actual geometric angle of attack varies greatly with radial position due to the complicated blade geometry.

As would be expected based on Equation 3.5, the measured cavitation extent on the propeller at a negative yaw angle reaches a maximum at an angular position of $\phi=180^{\circ}$. Also consistent is the fact that the data at lower advance ratios shows, on average, a greater cavitation extent. Finally, like the effective angle of attack, the cavitation extent at higher advance ratios shows a stronger variation with angular position.

Very interesting is that at higher advance ratios, the peak cavitation extent obtained at $\phi=180^{\circ}$ is equal to or greater than that obtained at lower advance ratios. This is surprising given that the effective angle of attack at this angular position is substantially 

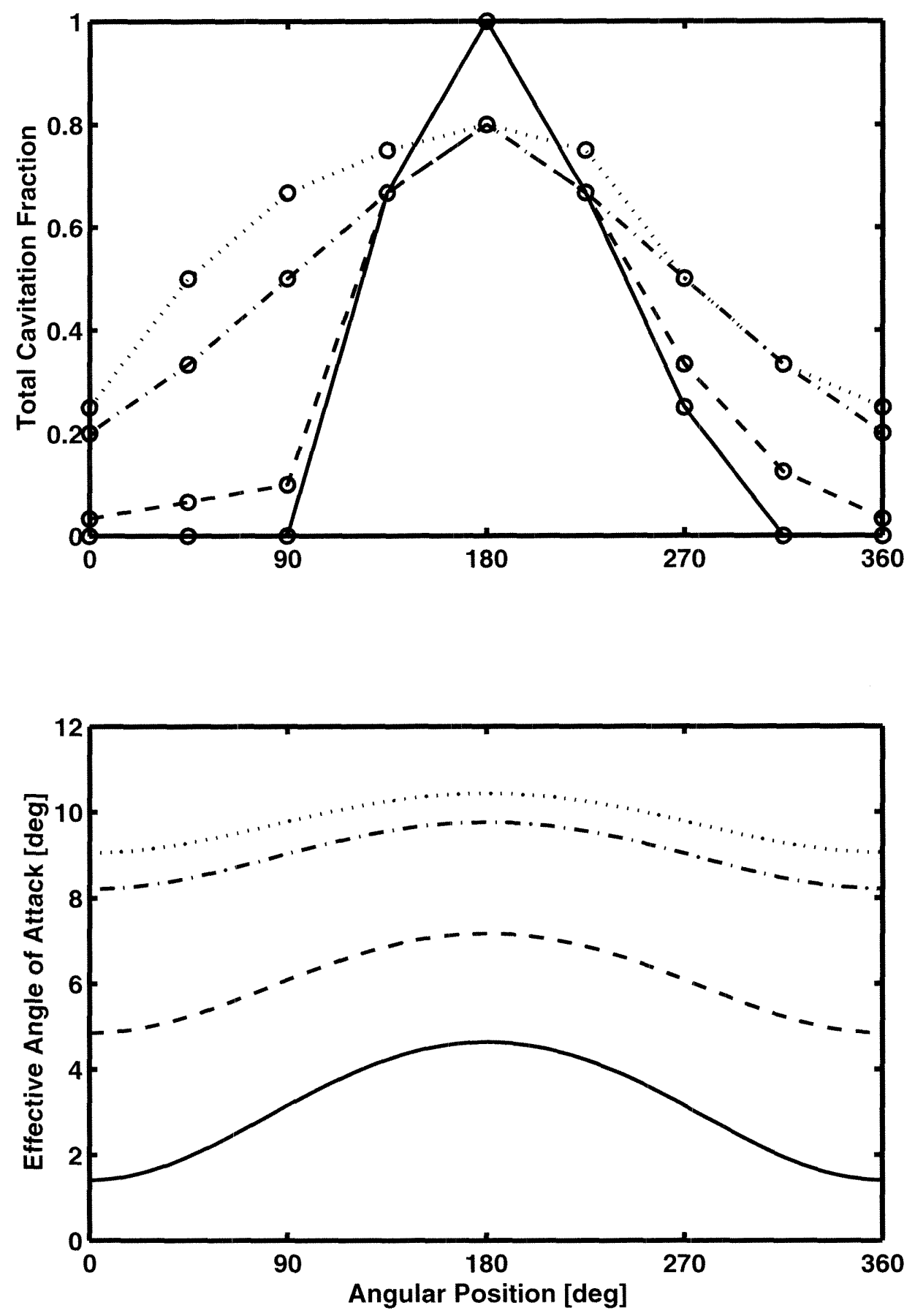

Figure 3.7: Cavitation extent for yawed propeller. Propeller operating at $\beta=-15^{\circ}$, $n=28.3 \mathrm{~Hz}$, and $\sigma=0.17$. Upper plot shows the total cavitation fraction $\frac{l_{t}}{c}$ at various angular locations (o). Lower plot shows the effective angle of attack $\alpha$ predicted by Equation 3.5 based on a geometric angle of attack of $\alpha_{o}=22^{\circ}$. Data and calculations are shown for $J=1.11(-), 0.93(--), 0.75(\cdot-\cdot)$, and $0.70(\cdots)$. 
lower at the higher advance ratios. Thus, while the average cavitation extent throughout the rotation and the angular location of the peak cavitation extent are accurately predicted by the effective angle of attack, the value of the peak cavitation extent is not. This strongly suggests that the cavitation extent does not simply track the varying effective angle of attack but is influenced by more complicated cavitation dynamics as well. The fact that the peak cavitation extent remains centered at the greatest effective angle of attack implies that any such dynamics must have rapid response times relative to the propeller rotation speeds.

\subsection{Unstable Cavitating Behavior}

\subsubsection{General Description of the Instability}

The experiments investigating the stable cavitating behavior of the propeller indicate that the extent of cavitation is largely but not fully described by the cavitation number and advance ratio. The yawed propeller experiments indicate that cavitation dynamics can lead to variations in cavitation extent beyond those predicted by an effective angle of attack calculation.

A more striking example of such variation in cavitation extent was observed when experiments were conducted with the propeller located downstream and therefore in the wake of the gear case. Under certain experimental conditions, an unstable behavior of the cavitation was observed. This instability was characterized by a periodic increase and decrease in the extent of cavitation both on the propeller blades and in the tip vortices shed downstream. This fluctuation in cavitation extent was readily apparent to the naked eye, and occurred evenly and synchronously on all blades and at all angular locations.

The frequency of the fluctuation was typically very steady, at a value in the range $f=9-11 \mathrm{~Hz}$. The instability was observed across a range of propeller rotation speeds of approximately $n=28.3-31.7 \mathrm{~Hz}$. However, in light of the effects of propeller rotation speed on the cavitating behavior of the propeller unaccounted for by the advance ratio and cavitation number, the majority of the experiments investigating the instability were conducted within a narrower range of rotation speeds of $n=30.0-30.8 \mathrm{~Hz}$. 


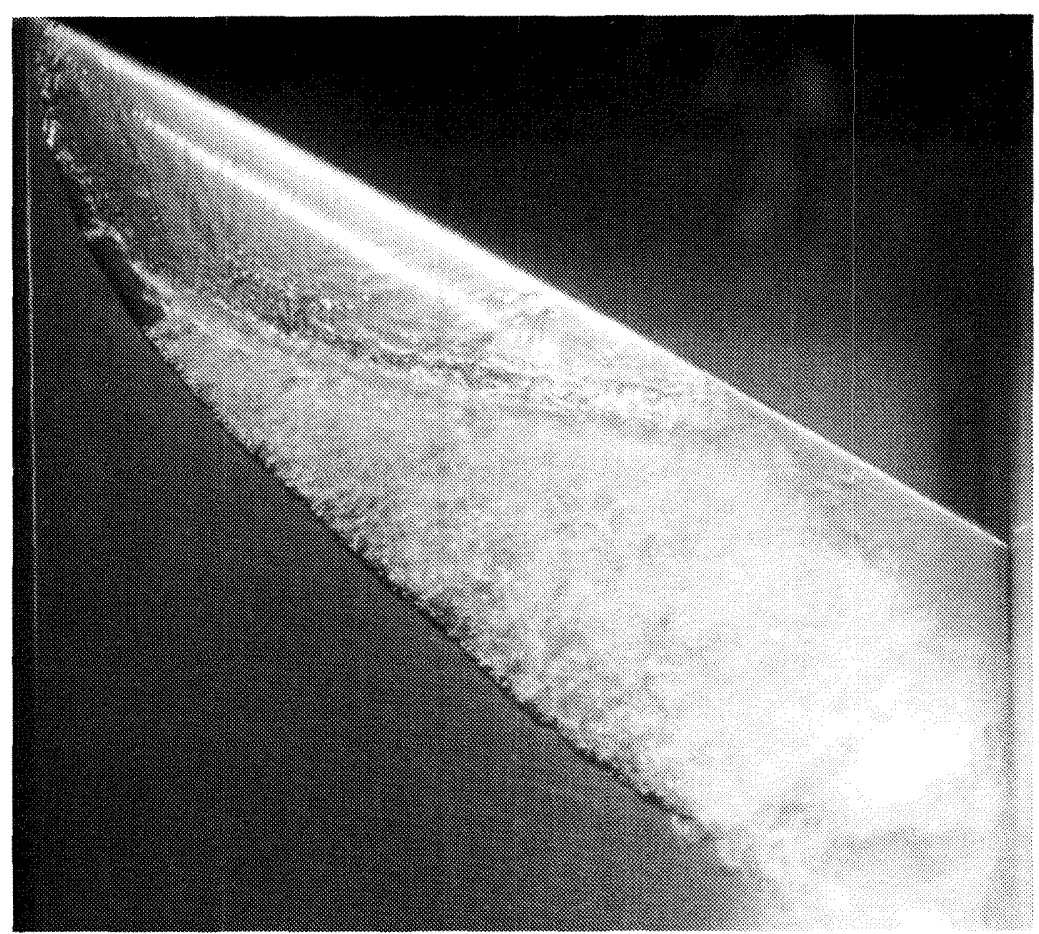

Figure 3.8: Photograph of re-entrant jet on three-dimensional hydrofoil. The suction surface of a hydrofoil spanning a water tunnel test section is shown with an incident flow from top to bottom in the photograph. The hydrofoil, with $30^{\circ}$ of sweep, is mounted at $\alpha=2^{\circ} . U=10.1 \mathrm{~ms}^{-1}$ and $\sigma=0.7$, based on the incident flow speed. Reproduced from Laberteaux and Ceccio (1998) with the authors' permission.

\subsubsection{Re-entrant Jet Behavior on Swept Hydrofoils}

While no record of an instability of this nature is found in the literature, preliminary visual inspection of the instability revealed similarities to observations made by previous investigators characterizing the cavitation on three-dimensional, swept hydrofoils. Figure 3.8 shows a photograph of the cavitation on a swept hydrofoil obtained by Laberteaux and Ceccio (1998). Figure 3.9 contains a diagram summarizing the structure of the cavitation. In the photograph and diagram, a region of clear cavitation attached to the leading edge is encroached upon by a structure referred to as a re-entrant jet, emanating from the cavity closure region. As this plume of water penetrates under the cavity from the line of closure, it strips the clear, attached cavitation from the surface of the hydrofoil and produces the bubbly cavitation present further downstream.

As can be seen from the photograph and diagram, the cavity closure line is not normal 


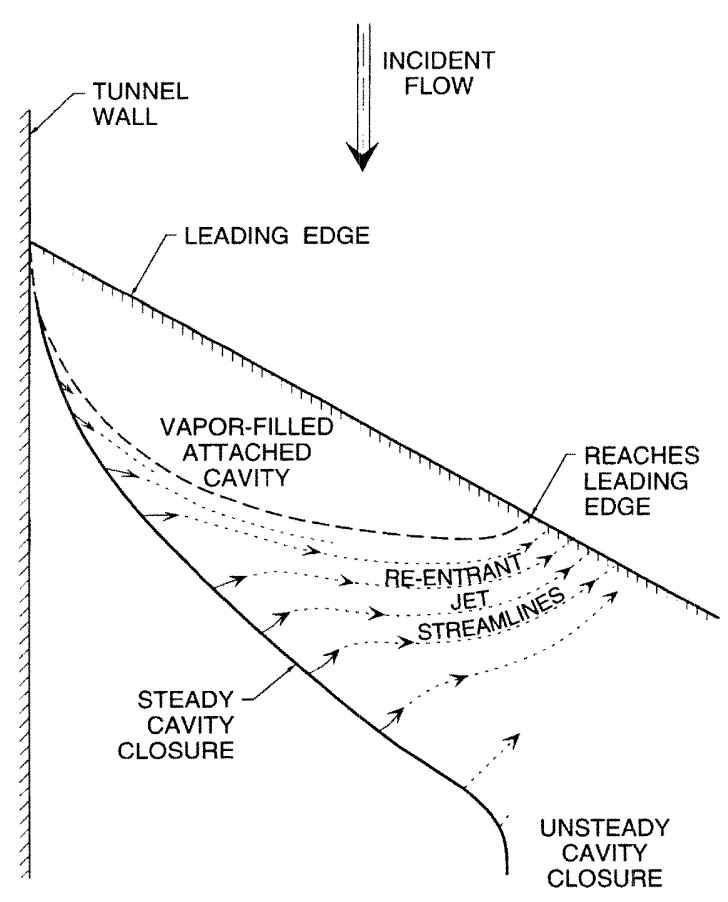

Figure 3.9: Schematic of re-entrant jet on three-dimensional hydrofoil. Shown are the orientation and evolution of the re-entrant jet for the flow over a three-dimensional swept hydrofoil, based on the photograph of Figure 3.8.

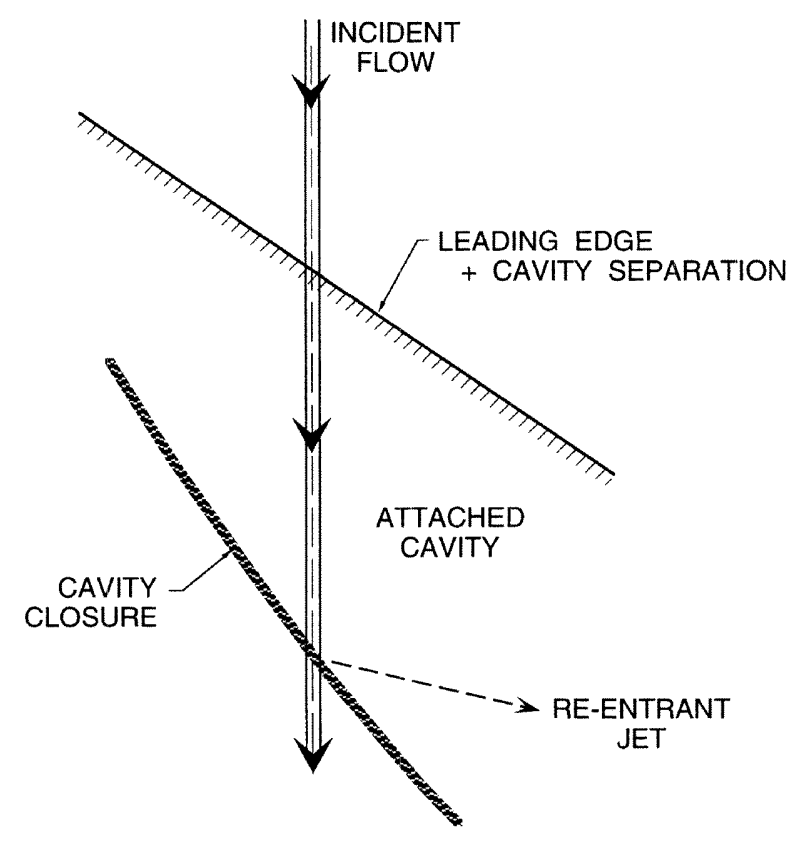

Figure 3.10: Schematic of reflection of re-entrant jet at a cavity closure. Shown is the reflection of a re-entrant jet at an inclined cavity closure. Adapted from de Lange, de Bruin, and van Wijngaarden (1994). 
to the direction of incoming flow, as would be the case for a two-dimensional hydrofoil, but rather is inclined as a result of the leading edge sweep. As indicated by de Lange, de Bruin, and van Wijngaarden (1994), in such geometries the re-entrant jet is not directed upstream but instead at a reflected angle from the line of cavity closure. This mechanism is detailed in Figure 3.10. Outboard of the point where the resulting re-entrant jet impinges upon the leading edge of the hydrofoil, very unsteady cavity closure is observed, in contrast to the clean and steady closure observed inboard of this point of impingement. A similar phenomenon has been noted in photographs of a swept hydrofoil obtained by Jessup (1997).

\subsubsection{Description of the Instability Cycle}

Figure 3.11 shows selected frames from high speed video footage of the instability on the cavitating propeller. The number indicated in each frame corresponds to the fraction, $\tau$, of the instability cycle elapsed. The beginning of the instability cycle is chosen arbitrarily to coincide with the minimal cavitation extent. This condition, as seen in the first frame of Figure 3.11 , is characterized by a relatively small region of bubbly cavitation along the leading edge of the propeller blade.

Further into the instability cycle, at $\tau=0.25$, the cavity has grown substantially towards the trailing edge of the propeller blade. A re-entrant jet is distinguishable as it is swept back from the leading edge, its position indicated by a white arrow. The spanwise location at which the re-entrant jet reaches the leading edge of the propeller blade is easily seen. As was observed by Laberteaux and Ceccio (1998), the cavity closure downstream and outboard of this location, in this case above and to the left, is very unsteady.

The cavity reaches its maximum extent at approximately $\tau=0.70$. At this stage of the instability cycle, a supercavitating condition is obtained when the cavity near the tip of the propeller blade extends rearward past the trailing edge of the blade. As this cavitation is entrained into the tip vortices it leads to a readily observed increase in the diameter of the vortices shed downstream of the propeller. No re-entrant jet is visible, presumably because the jet fluid has been swept past the cavity closure region.

By $\tau=0.92$ the cavitation has begun to decrease in extent, receding towards the 

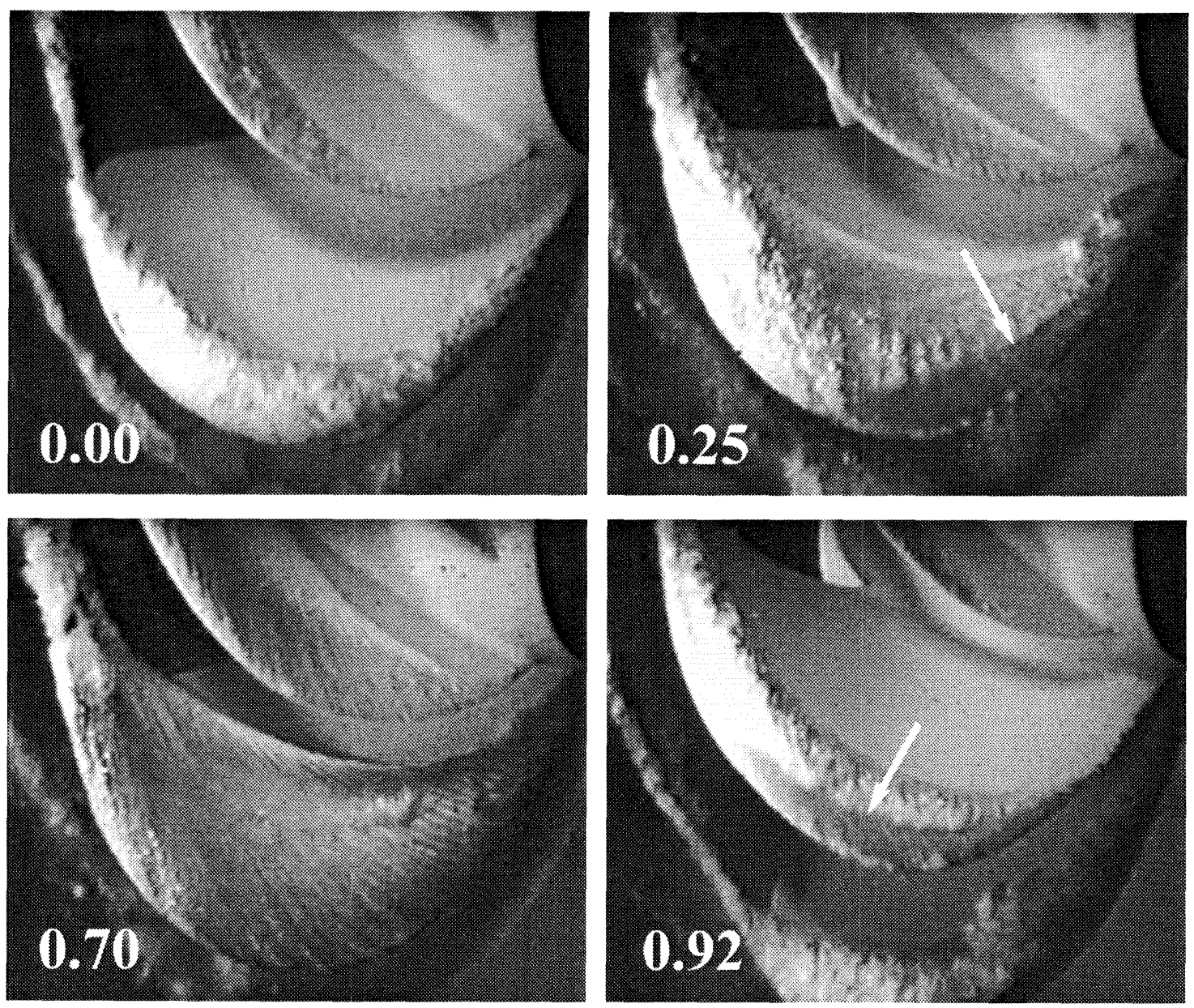

Figure 3.11: Cavitation variation during instability cycle. Presented is a sequence of sequential but not consecutive frames taken from high speed video footage, showing the variation in cavitation seen on an individual propeller blade during the instability cycle. The number in the lower left corner of each frame, $\tau$, indicates the fraction of the instability cycle elapsed.

leading edge of the propeller blades. The re-entrant jet can again be observed, indicated by the white arrow, as it begins its rush forward towards the leading edge. When the re-entrant jet reaches the leading edge at nearly all spanwise locations, the cavitation returns to the minimal configuration shown at $\tau=0.00$.

\subsubsection{Similarities to the Partial Cavity Instability}

The preceding description suggests several similarities between the instability cycle and the well documented partial cavity instability observed on two-dimensional hydrofoils 
(Wade and Acosta 1966; Franc and Michel 1988; Le, Franc, and Michel 1989; de Lange, de Bruin, and van Wijngaarden 1994). First, the cavity length on the propeller blade is fluctuating between two very different but consistent values. Furthermore, the cavity lengths about which the fluctuation occurs are comparable to the chord length of the propeller blade. Finally, the frequency of the fluctuation is quite low. Wade and Acosta (1966) reported reduced frequencies, $k=\frac{f c}{U}$, based on chord length, $c$, and incoming flow velocity, $U$, in the range $k=0.07-0.14$. Other investigators (Le, Franc, and Michel 1989; de Lange, de Bruin, and van Wijngaarden 1994) encountered somewhat higher values of approximately $k=0.34$. If a reduced frequency for the propeller instability is computed based on the chord length and incident velocity at the effective radius, a value of $k=0.07$ is obtained, consistent with the range reported by Wade and Acosta (1966), but somewhat lower than the value reported by the other investigators. The precise significance of this is not clear, though, as the mechanism that determines the frequency of the partial cavity instability is not understood.

\subsubsection{Measurement of the Pressures Generated by the Instability}

During experiments when the instability was present, the resulting fluctuating pressures were strong enough to be readily heard in the laboratory and to shake the test section violently. The pressure transducers shown in Figure 2.4 were utilized to quantify these fluctuating pressures. A typical signal obtained from the floor-mounted pressure transducer is shown in Figure 3.12. The signal is clearly periodic, with a frequency of approximately $f=10 \mathrm{~Hz}$, corresponding to the frequency of cavitation variation observed in the high speed video footage. The magnitude of the pressure fluctuations is a remarkable $15 \mathrm{kPa}$. The amplitude seen in similar pressure signals varies greatly, even among those obtained at identical operating conditions. However, it is clear from direct visual observation that the largest pressure amplitudes are obtained during the most dramatic fluctuations in cavity extent.

Figure 3.13 shows the power spectral density of the pressures generated by the instability. This spectrum was calculated by averaging the power spectral densities computed for several floor mounted transducer signals similar to that shown in Figure 3.12. Clearly 


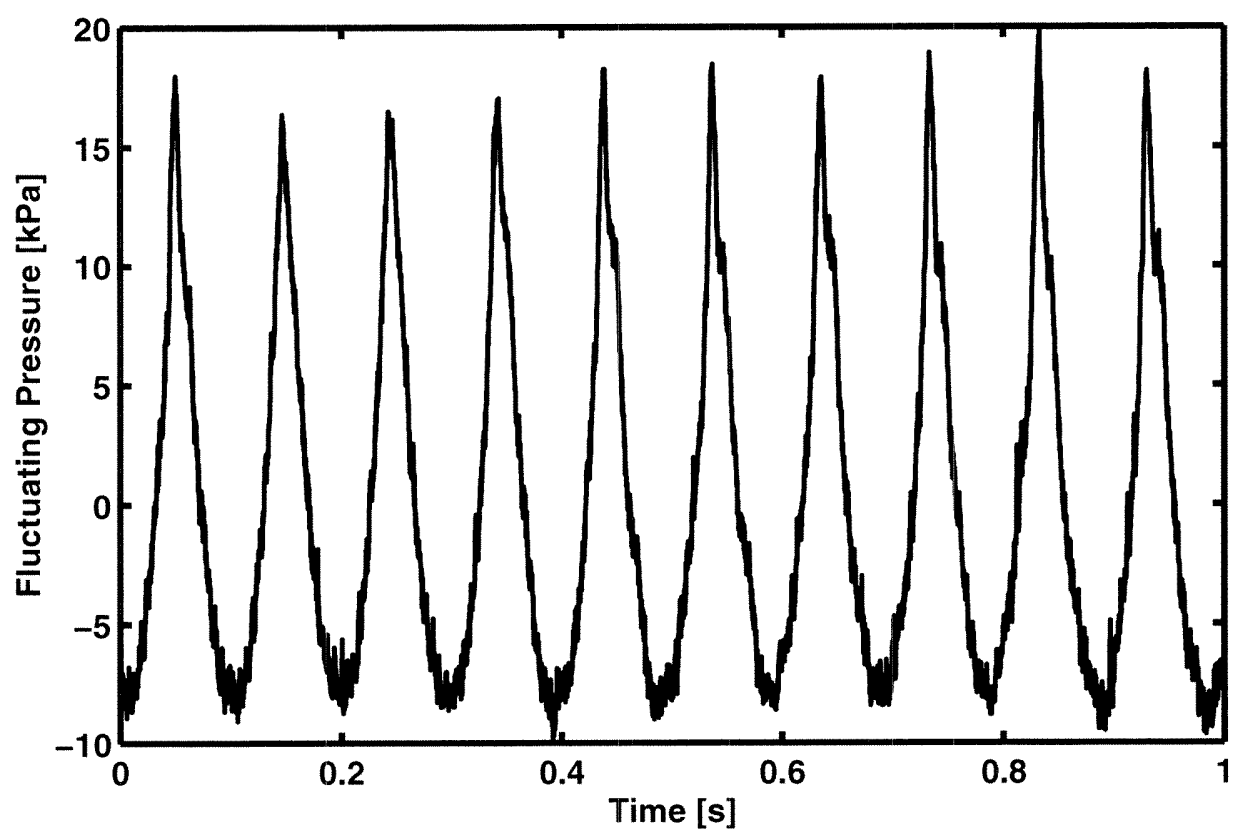

Figure 3.12: Measured pressures generated by instability. The pressure measurements shown were obtained from the floor-mounted pressure transducer. The signal was lowpass filtered at $2 \mathrm{kHz}$.

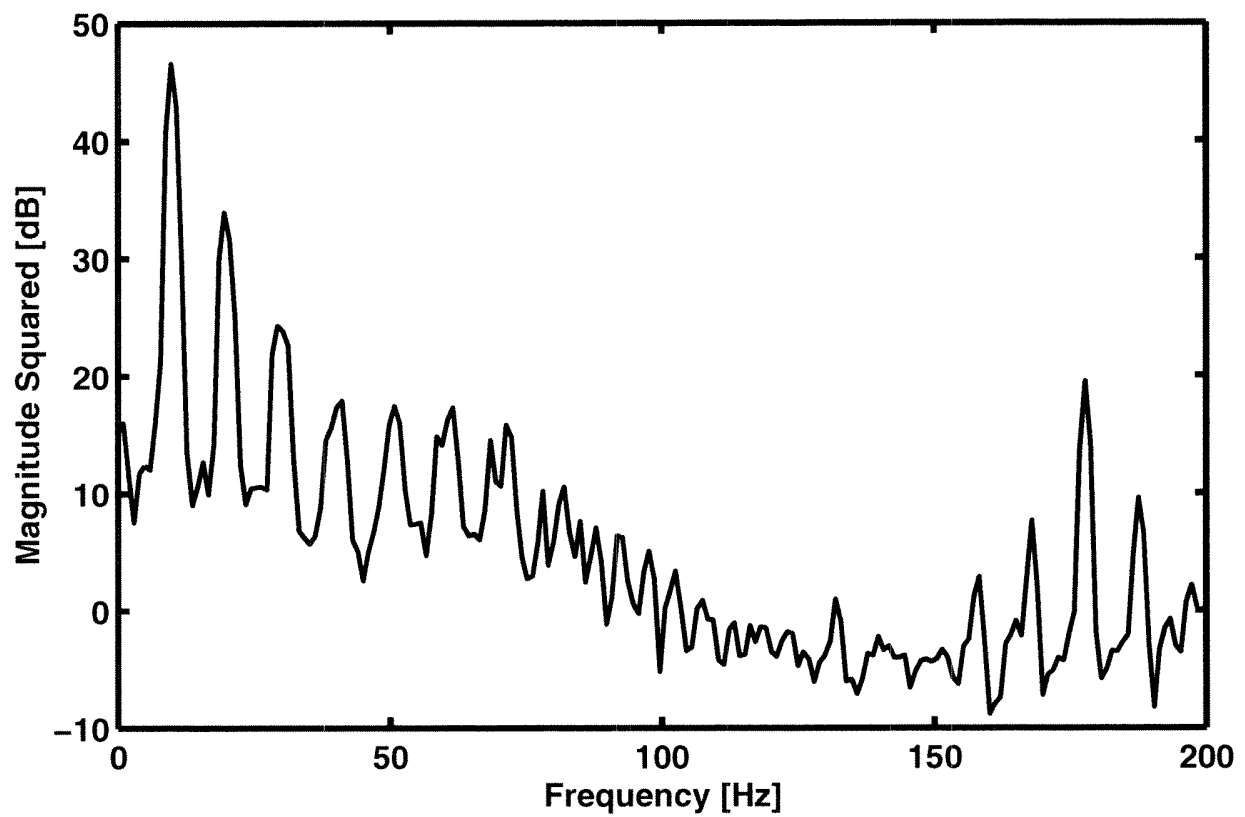

Figure 3.13: Power spectral density of pressures generated by instability. Shown is an average power spectral density of several signals obtained from the floor-mounted pressure transducer. 
visible at approximately $10 \mathrm{~Hz}$ is the fundamental frequency of the instability, dominating the instability noise by a margin of approximately $15 \mathrm{~dB}$. Many harmonics can also be seen. At $180 \mathrm{~Hz}$, the blade passage frequency can be identified, corresponding to the six bladed propeller rotating at $n=30.0 \mathrm{~Hz}$. Finally, to either side of the blade passage frequency, the beat between the blade passage frequency and the fundamental instability frequency can be discerned.

\subsubsection{Onset of the Instability}

Determining the precise range of conditions under which the instability occurs posed a substantial challenge. The fluctuations in cavitation extent were not always as dramatic as those shown in Figure 3.11 and the fluctuating pressures were not always audible or as easily distinguished as in Figure 3.12. In reality, the behavior of the cavitating propeller is not easily classified as purely stable or unstable, but is instead better described by a continuum of unstable behavior of varying intensity. These difficulties were compounded by the fact that once a set of operating conditions was achieved within the facility, the unstable behavior would often only develop after a delay of 1 or $2 \mathrm{~min}$.

Ultimately, the most reliable criterion for classifying the behavior of the propeller as stable or unstable was found by analyzing the pressure measurements of the floor-mounted pressure transducer. Figure 3.14 presents a series of power spectral densities obtained from this transducer. Shown is the evolution of the spectra as the cavitation number is reduced at a fixed advance ratio. As the cavitation number is reduced, the unstable behavior increases in intensity. This is characterized by a transition in the dominant frequency, from a value near $f=14 \mathrm{~Hz}$, to a value near $f=9 \mathrm{~Hz}$. This growth of the lower frequency peak was utilized in defining the onset of instability. Following this onset, the frequency of the dominant peak remained constant with increasing intensity of the instability.

Figure 3.15 summarizes the results of experiments investigating the onset of the instability in the parameter space defined by the cavitation number and advance ratio. Analysis of the power spectral density at each operating condition allowed for the classification of each point as either stable or unstable. Operating conditions where successive 

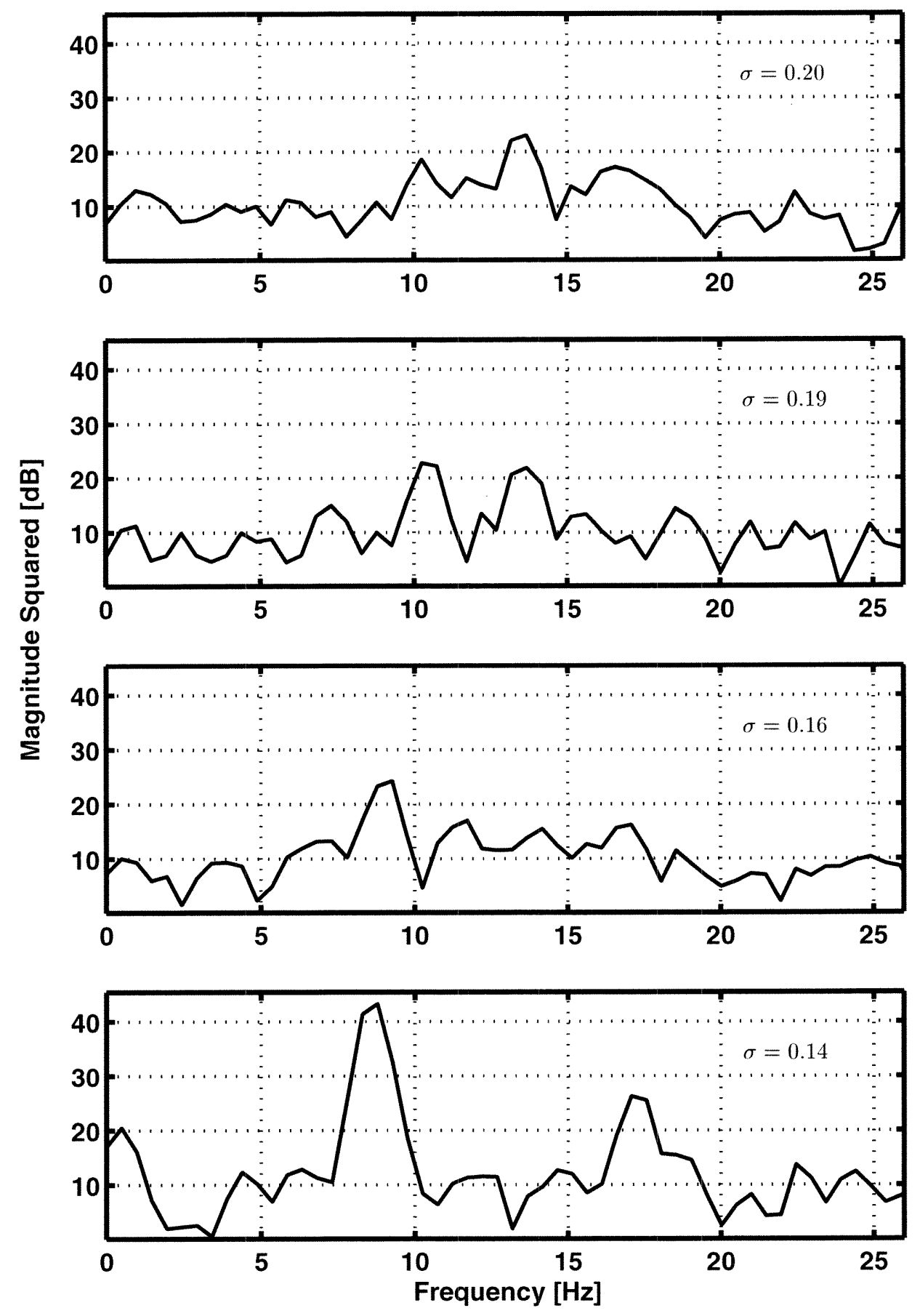

Figure 3.14: Series of power spectral densities at decreasing cavitation number. The series of plots was obtained at decreasing cavitation number, with a constant advance ratio of $J=0.72$. The propeller rotation speed was $n=30.0 \mathrm{~Hz}$. 


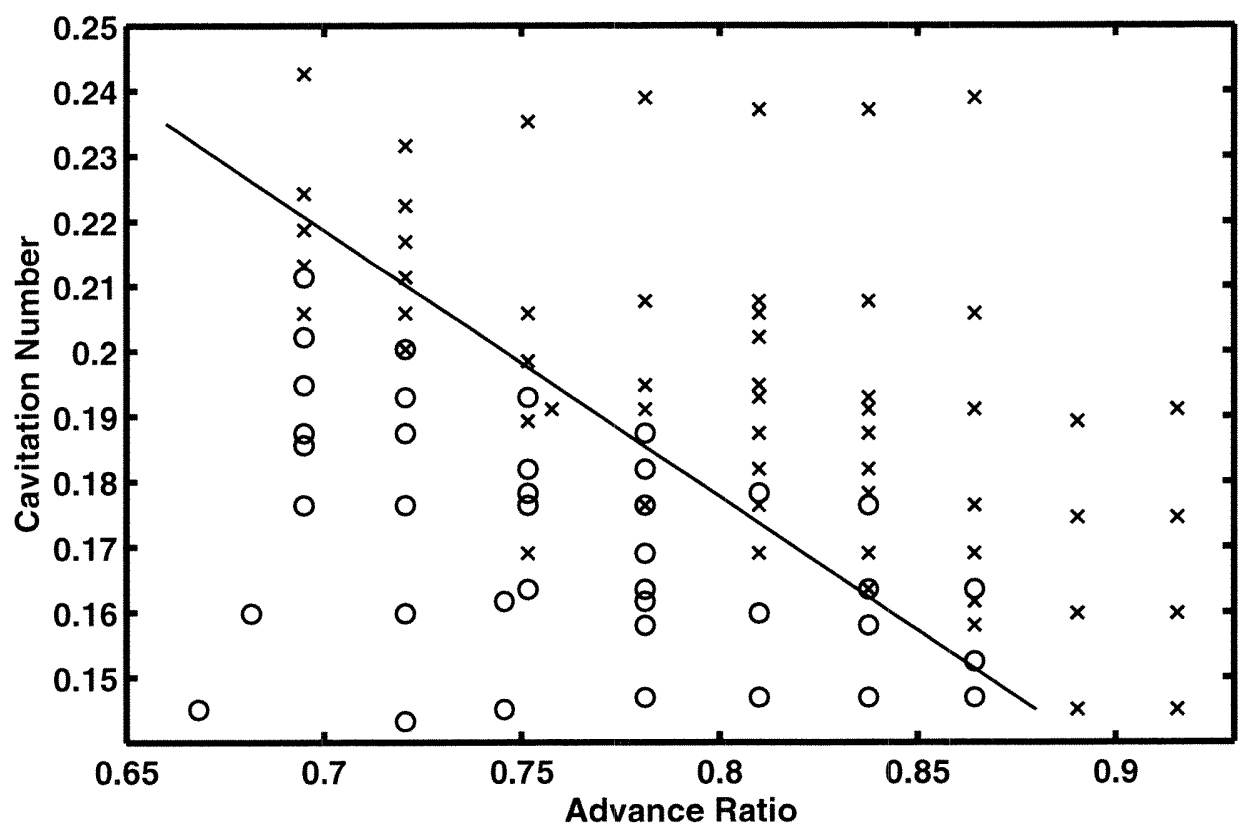

Figure 3.15: Onset of instability with cavitation number and advance ratio. Each experimental operating point is classified as stable $(\times)$, unstable $(0)$, or marginally stable $(\otimes)$ according to the criterion described in Figure 3.14. The propeller rotation speed was $n=30.8 \mathrm{~Hz}$.

tests exhibited stable and unstable behavior are classified as marginally stable. Clearly, the unstable operating conditions are concentrated at lower cavitation numbers and at advance ratios below the design advance ratio of the propeller, $J_{o}=1.15$. Because cavitation numbers below $\sigma=0.14$ could not be obtained in the experimental facility, it could not be determined whether or not the instability occurs at the design advance ratio. Finally, the solid line represents an attempt to divide the stable and unstable operating conditions, and may be considered the experimentally determined onset of instability.

Some insight regarding the onset of the instability can be gained by considering the following argument. Consistent with the reasoning of Section 3.1, early studies (Tulin 1953 ) showed that the non-dimensional cavity length, $\frac{l}{c}$, on a two-dimensional hydrofoil is essentially a function of the ratio $\frac{\alpha}{\sigma}$. Furthermore, from Equation 3.3 it can be shown that the angle of attack of a propeller blade is approximately proportional to the difference, $J_{o}-J$, between the design and operating advance ratios. Thus a particular configuration of cavity lengths on a propeller blade should correspond to a particular value of the 


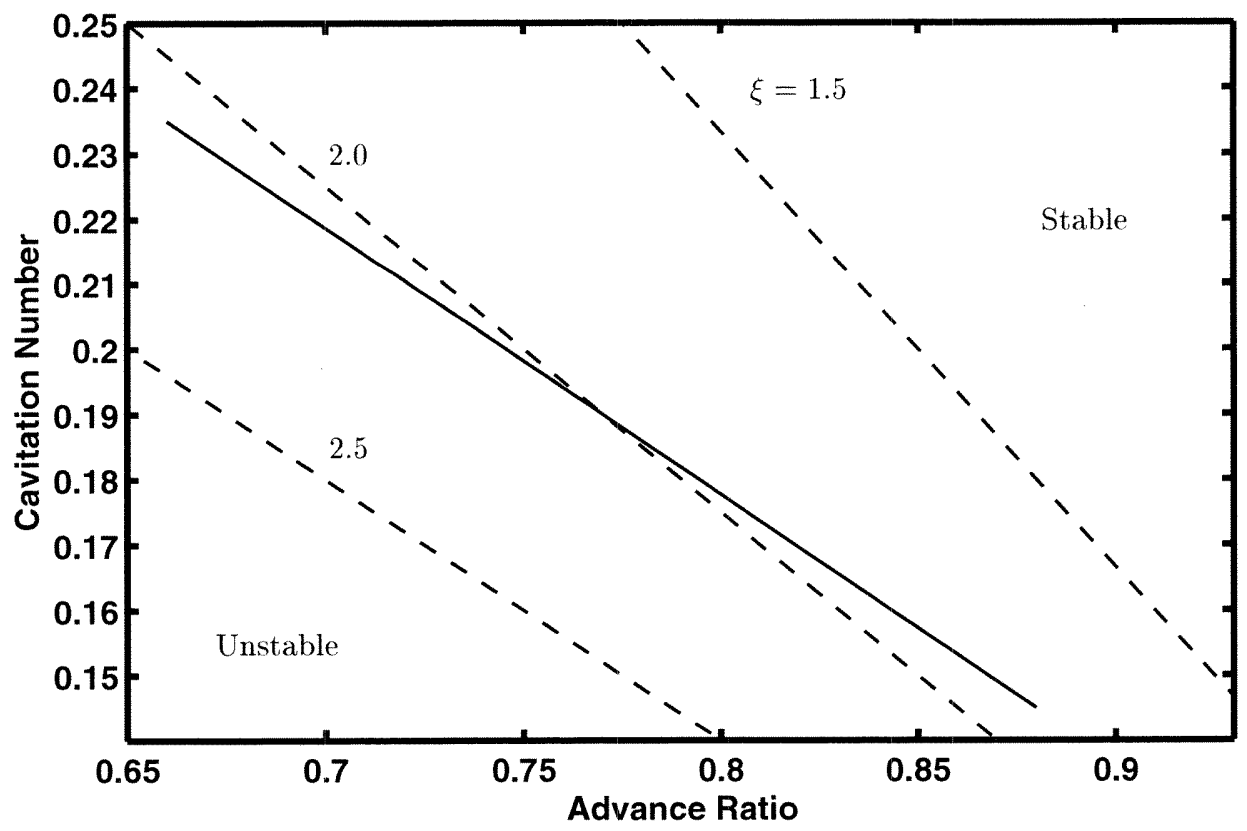

Figure 3.16: Comparison of instability onset with stability criterion based on dimensionless cavity length. The solid line $(-)$ shows the experimentally determined onset of stability, determined in Figure 3.15. The dashed lines (--) correspond to the stability criterion based on cavity length, for several different values of the parameter $\xi$.

parameter $\xi=\frac{J_{0}-J}{\sigma}$.

Several lines of fixed $\xi$ are plotted in Figure 3.16. The experimentally determined instability boundary corresponds quite closely to the particular value of $\xi=2.0$, suggesting that the instability boundary corresponds to a particular configuration of cavity lengths on the propeller blade. This reaffirms the connection with the partial cavity instability observed on two-dimensional hydrofoils, where stability was also related to the non-dimensional cavity length.

\subsubsection{Variation of the Instability Frequency}

An important fact yet unaddressed is that the instability was only observed during experiments conducted with the propeller mounted downstream of the gear case. Any attempt to determine the cause of the instability and in particular the frequency of the instability must begin by considering the effects of the gear case wake on the cavitating propeller. More specifically, the periodicity of any vortex shedding occurring in the gear case wake 
seems a possible source of the periodicity of the fluctuation in cavitation extent.

A crude approximation for the frequency of such vortex shedding can be estimated by approximating the gear case as cylindrical in shape, and evaluating the Strouhal frequency of this cylinder. Across a range of Reynolds numbers from Re $=1 \times 10^{2}$ to $\operatorname{Re}=1 \times 10^{5}$, it is known that

$$
\mathrm{St}=\frac{f D}{U_{t}}=0.2
$$

where $f$ is the frequency of vortex shedding, $D$ the cylinder diameter, and $U_{t}$ the test section flow velocity. Of course, choosing an appropriate value for the diameter of the cylinder is difficult given the irregular shape of the gear case. However, a range of diameters can be considered, with a maximum diameter of $D=65 \mathrm{~mm}$ based on the width of the gear case at the height of the propeller axis, and a minimum diameter of $D=5 \mathrm{~mm}$ based on the width of the vane above the propeller axis. For a typical test section flow velocity during the instability experiments of $U_{t}=3.75 \mathrm{~ms}^{-1}$, these two diameters yield vortex shedding frequencies of $f=11.2 \mathrm{~Hz}$ and $f=150 \mathrm{~Hz}$, respectively. The corresponding Reynolds numbers for the two diameters are $\operatorname{Re}=2.01 \times 10^{5}$ and $\operatorname{Re}=1.55 \times 10^{4}$, only slightly out of the accepted range of validity for the Strouhal frequency. The estimated frequency range differs substantially from the observed frequencies of the instability. But given that the propeller disc is more closely in line with the maximum diameter than the minimum diameter, the possibility of vortex shedding defining the instability frequency cannot be completely discounted.

Further insight can be gained by considering the variation in the instability frequency with changes in flow conditions. Figures 3.17 and 3.18 show the variation in instability frequency with changes in advance ratio and cavitation number, respectively. The frequencies were determined by correlating the ceiling-mounted pressure transducer measurements with complex fluctuating signals of various frequencies. Unfortunately, only a narrow range of cavitation numbers and advance ratios could be explored, as consistently determining the frequency of the instability proved possible only at operating conditions well within the interior of the instability region defined in Figure 3.15. 


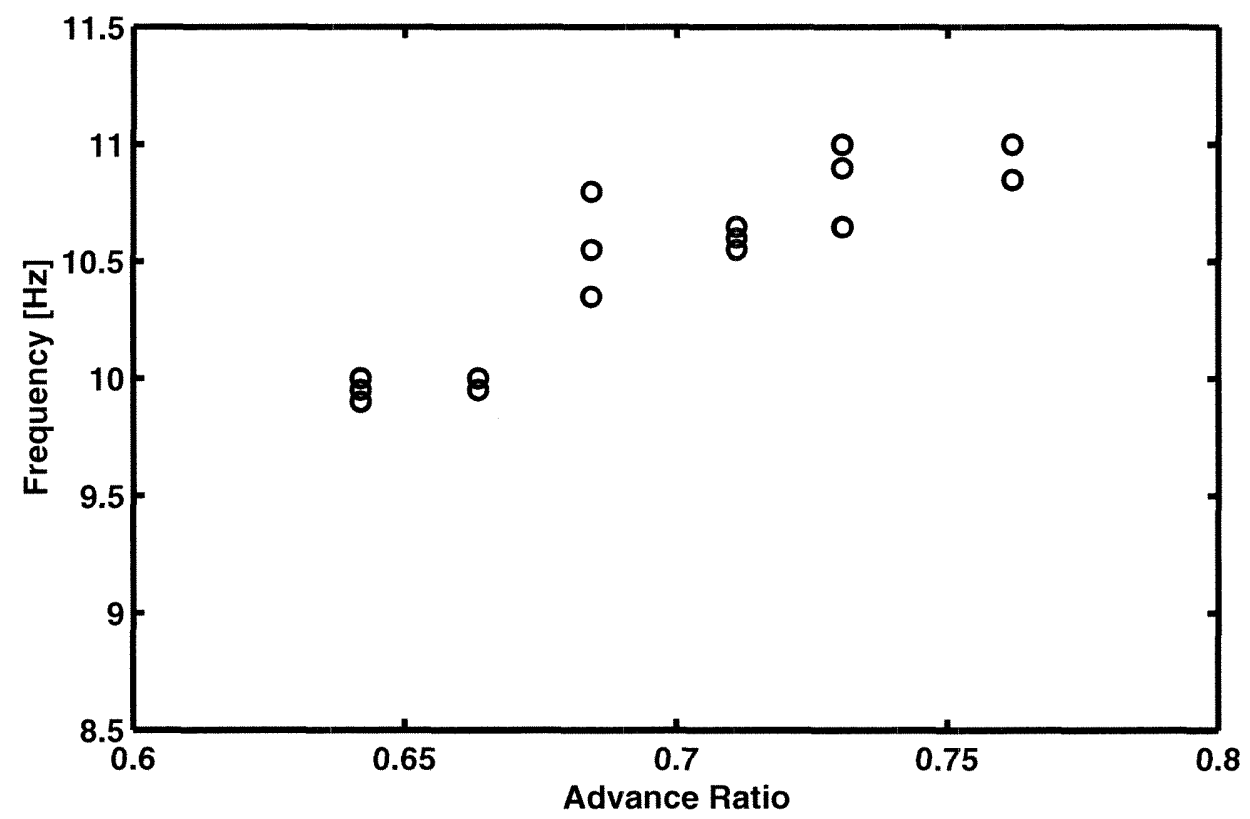

Figure 3.17: Variation in instability frequency with advance ratio. Shown are experimentally determined instability frequencies $(0)$ obtained at a propeller rotation speed of $n=30.4 \mathrm{~Hz}$ and a constant cavitation number of $\sigma=0.15$.

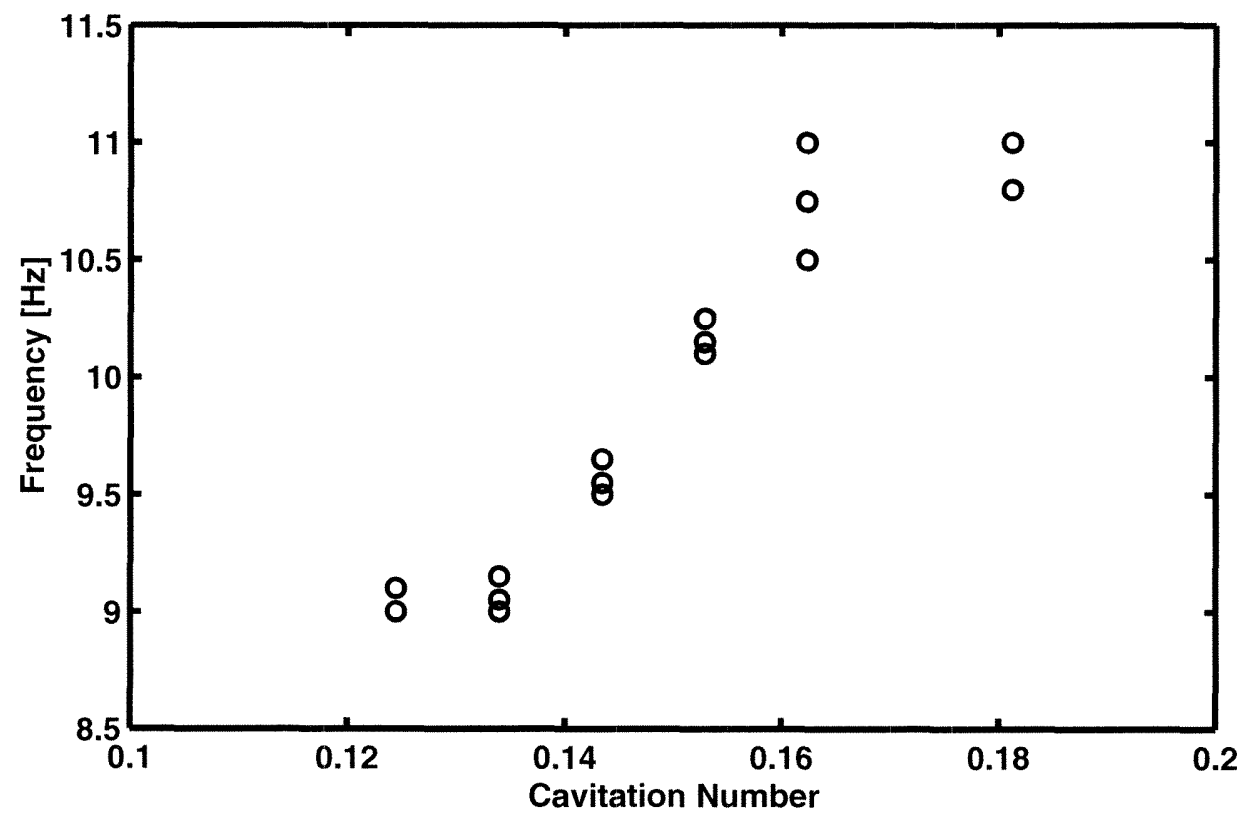

Figure 3.18: Variation in instability frequency with cavitation number. Shown are experimentally determined instability frequencies $(0)$ obtained at a propeller rotation speed of $n=30.4 \mathrm{~Hz}$, and a constant advance ratio of $J=0.64$. 
Nonetheless, significant trends are apparent in both figures across the range of parameters explored. Figure 3.17 shows a significant increase in the instability frequency with increasing advance ratio. Given that the advance ratio is varied by changing the test section flow velocity, $U_{t}$, this change in frequency is consistent with the behavior of the vortex shedding frequency given by Equation 3.6. In fact, the dependence of the instability frequency on the advance ratio is approximately linear, with an intercept near the origin, also consistent with Equation 3.6. This lends support to the notion that the instability frequency is determined by vortex shedding from the gear case.

Figure 3.18 shows an even more dramatic trend of increasing instability frequency with increasing cavitation number. However, because these experiments were conducted at a constant test section flow velocity, it is not possible to explain this trend with variations in vortex shedding frequency. Also, given the irregular shape of the gear case, it would seem likely that certain regions of the propeller disc would be exposed to more dramatic vortex shedding than others. This is inconsistent with the uniform and synchronous nature of the fluctuations in cavitation extent. Moreover, the gear case, with a streamlined shape that is far from cylindrical, is presumably designed to minimize vortex shedding at all locations. In light of these considerations, it appears that a mechanism other than vortex shedding determines the instability frequency.

\subsubsection{Analysis of the Pressures Generated by the Instability}

\section{Volumetric Acceleration}

To better understand the pressure fluctuations observed in Section 3.3.5, a simple model can be constructed for the pressure generated by the fluctuating cavitation extent and therefore fluctuating cavity volume of the instability. As suggested by Huse (1972), the far field pressure from a fluctuating cavity volume will be dominated by the volumetric acceleration imposed upon the flow. Specifically,

$$
\tilde{p} \sim \frac{d^{2} \tilde{V}_{c a v}}{d t^{2}}
$$




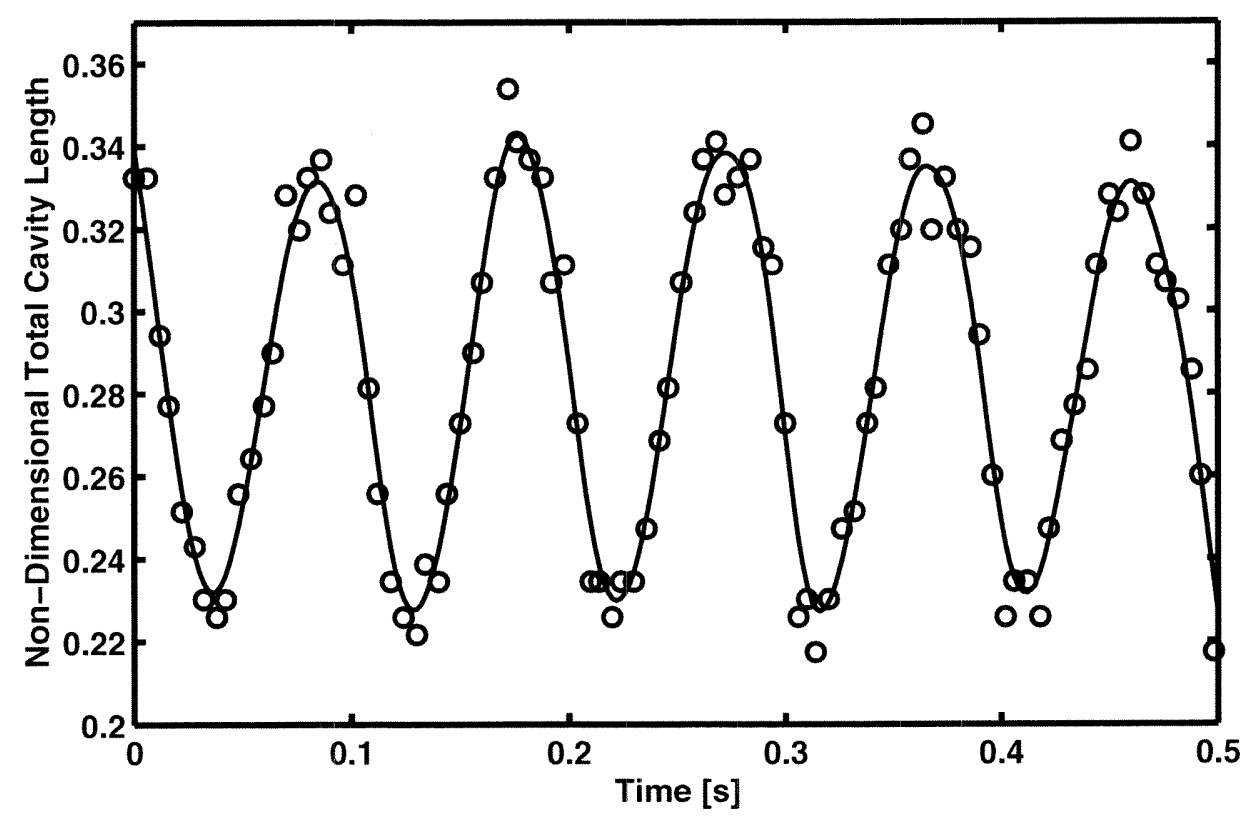

Figure 3.19: Non-dimensional cavity length through several instability cycles. The quantity $\frac{l_{t}}{c}$ is shown for several cavity volume fluctuation cycles, as determined from the high speed video footage. The direct measurements $(0)$ are smoothed and interpolated $(-)$.

where $\tilde{p}$ is the fluctuating far field pressure and $\tilde{V}_{c a v}$ is the fluctuating cavity volume. This volumetric acceleration can be determined from direct measurements of the cavity volume throughout the instability cycle.

To obtain these measurements, high speed video footage similar to that shown in Figure 3.11 was obtained, and synchronized by a timing pulse with measurements from the floor-mounted pressure transducer. Sequential but not necessarily consecutive frames of the video footage were then digitally captured and analyzed on a frame by frame basis. In each case, a single measurement of the non-dimensional total cavity length, $\frac{l_{t}}{c}$, was obtained as illustrated in Figure 3.3. These cavity length measurements were then smoothed, interpolated, and matched to the time line of the floor-mounted pressure transducer measurements. The resulting variations in cavity length are shown in Figure 3.19. The particular instance of the instability captured was relatively mild, with an amplitude of non-dimensional cavity length fluctuations of approximately $\frac{c}{10}$, and a fluctuating pressure amplitude of approximately $6 \mathrm{kPa}$.

From these cavity length measurements, an estimate of the cavity volume was obtained 
by following the suggestion of Blake (1986) that $V_{\text {cav }} \sim R l_{t}^{2}$, where $R$ is the radial cavitation extent and $l_{t}$ the total chordwise cavitation extent determined from the video footage. Essentially, this relationship assumes that the cavity volume maintains a constant radial extent and exhibits geometric similarity throughout the instability cycle, with the cavity thickness always proportional to the cavity extent.

Guided by direct visual observation of the instability cavitation and the findings of Laberteaux and Ceccio (1998), the cavity thickness was assumed to be one-eighth of the cavity extent. Approximating the cavity to be roughly triangular in cross-section, the cavity volume was then evaluated as

$$
V_{c a v}=\frac{N}{16}\left(R-R_{h u b}\right) l_{t}^{2}
$$

where $N$ is the number of propeller blades. It should be repeated that this expression gives only a rough approximation of the cavity volume, due to the assumptions outlined above and the fact that it does not take into account any spanwise variations in the cavity length.

A finite difference method applied to the cavity volume estimates was then used to determine the second derivative of cavity volume and therefore the volumetric acceleration imposed upon the flow. Figure 3.20 shows the results of this calculation, compared with the pressure measurements obtained from the floor-mounted transducer. As Equation 3.7 offers no indication of the amplitude of the far field pressure fluctuations, the vertical scale of Figure 3.20 is chosen arbitrarily to facilitate comparison of the shape of the two signals. Very clearly, the two signals correspond closely in terms of period and phase. Furthermore, the volumetric acceleration curve tracks remarkably well some of the more subtle features in the troughs of the floor-mounted transducer signal.

\section{Amplitude of the Fluctuating Pressures}

To estimate the amplitude of the pressures generated by the instability, a model is needed to describe the propagation of the fluctuating pressures into the fluid surrounding the propeller. There are several different approaches that may be adopted. One possible 


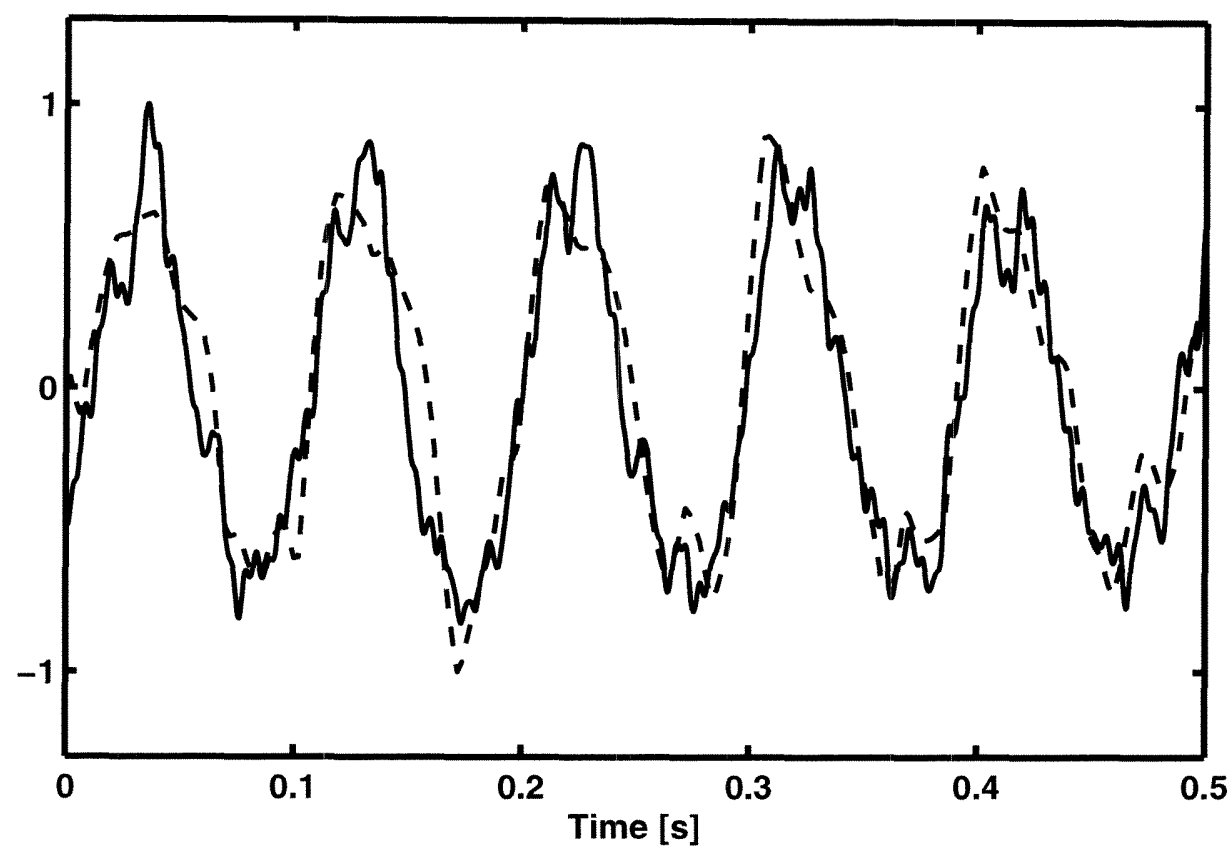

Figure 3.20: Comparison of volumetric acceleration and measured pressure. The solid line (-) shows the floor-mounted pressure transducer signal, low-pass filtered at $200 \mathrm{~Hz}$. The dashed line (--) shows the results of the finite difference method second derivative calculation. The vertical axis is scaled to facilitate comparison.

approach is a three-dimensional model that treats the propeller cavitation as a point source of fluctuating pressure radiating into an infinite field. A second possible approach is a one-dimensional model that treats the propeller cavitation as a roughly planar source of fluctuating pressure, spanning the entire cross-section of the tunnel test section. The resulting flow within the facility is then essentially one-dimensional, and the propagation of the fluctuating pressure is determined by the dynamic behavior of the various components of the surrounding facility. Finally, a combination of these two approaches could be used, incorporating the three-dimensional approach at locations very near the propeller and employing the one-dimensional approach at greater distances.

The validity of the three-dimensional approach can be readily assessed from the data of Figure 3.20. If a three-dimensional model is adopted, the amplitude of the pressure fluctuations decays with increasing distance, and is given by (Brennen 1995)

$$
\tilde{p}=\frac{\rho}{4 \pi \mathcal{R}} \frac{d^{2} \tilde{V}_{c a v}}{d t^{2}}
$$




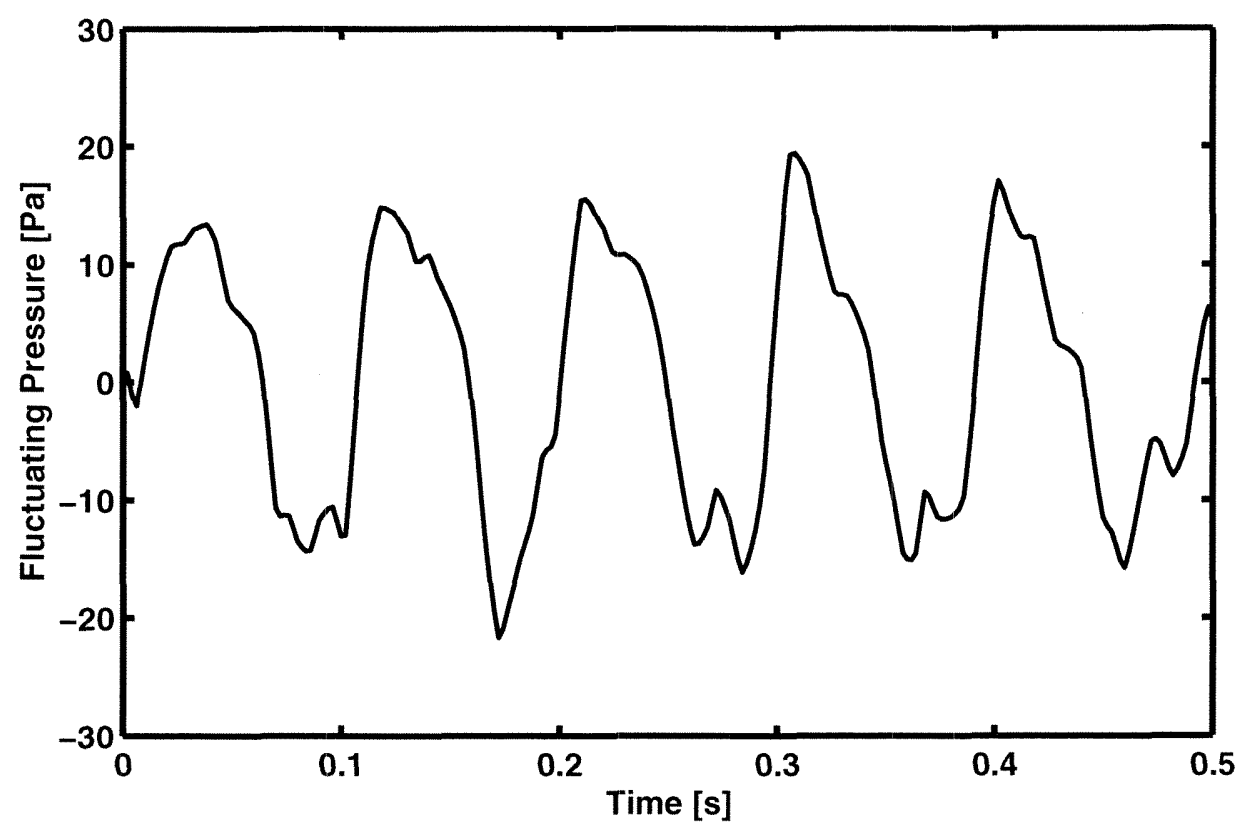

Figure 3.21: Predicted fluctuating pressure at floor-mounted transducer. Shown is the fluctuating pressure at the floor-mounted transducer predicted by Equation 3.9.

where $\mathcal{R}$ is the distance from the point of pressure measurement to the center of the fluctuating cavity volume.

In the case of the cavitating propeller, it is difficult to infer a precise value for $\mathcal{R}$, as there is not a single cavity, but rather one cavity on each propeller blade, each of which is complex in shape. It is therefore helpful to assume that the collective cavity center is located along the axis and in the plane of blade rotation. Then, distances of $\mathcal{R}_{f}=0.15 \mathrm{~m}$ and $\mathcal{R}_{c}=0.43 \mathrm{~m}$ are obtained for the floor- and ceiling-mounted transducers of Figure 2.5. Applying the value of $\mathcal{R}_{f}$ to Equation 3.9 yields the fluctuating pressure shown in Figure 3.21. The resulting amplitude of the pressure fluctuations, approximately $15 \mathrm{~Pa}$, is several orders of magnitude less than the $6 \mathrm{kPa}$ observed experimentally.

The far field pressure can be estimated more generally by assuming that the cavity volume varies sinusoidally according to the relation

$$
V_{c a v}=\bar{V}_{c a v}+\left|\tilde{V}_{c a v}\right| \sin (\omega t)
$$

where $\left|\tilde{V}_{c a v}\right|$ is the amplitude of the volumetric excitation. Video footage similar to that 
of Figure 3.11 suggests that during the instability cycle the cavity typically fluctuates between non-dimensional cavity lengths of $\frac{l_{t}}{c}=\frac{1}{5}$ and $\frac{l_{t}}{c}=\frac{2}{3}$, a larger fluctuation than observed in Figure 3.19. From Equation 3.8, with $c=88.0 \mathrm{~mm}$, this implies an amplitude of volumetric fluctuation of $\left|\tilde{V}_{c a v}\right|=4.20 \times 10^{-5} \mathrm{~m}^{3}$. Noting that the magnitude of the volumetric acceleration is given by

$$
\left|\frac{d^{2} \tilde{V}_{c a v}}{d t^{2}}\right|=\omega^{2}\left|\tilde{V}_{c a v}\right|
$$

this yields fluctuating pressures at the floor- and ceiling-mounted transducers of $\left|\tilde{p}_{f}\right|=$ $88 \mathrm{~Pa}$ and $\left|\tilde{p}_{c}\right|=31 \mathrm{~Pa}$ for a typical instability frequency of $f=10 \mathrm{~Hz}$. These values compare extremely poorly with those measured experimentally, which, as evidenced by Figure 3.12 , are often as high as $15 \mathrm{kPa}$.

These results demonstrate that the three-dimensional model alone is wholly inadequate in explaining the far field pressures of the instability. Even at distances as close to the propeller as the floor- and ceiling-mounted transducers, the flow is no longer adequately modeled by a three-dimensional approach. This suggests that to fully understand the fluctuating pressures generated by the instability, a one-dimensional model incorporating the characteristics of the surrounding facility is needed. 


\section{Chapter 4}

\section{Experimental Facility Dynamics}

The experimental observations presented in Chapter 3 document a newly observed instability on a cavitating propeller. The nature of the cavitation throughout the instability cycle was noted, the conditions under which the instability occurs were determined, and the transition from stable to unstable behavior was found to be similar to that of the partial cavity instability on two-dimensional hydrofoils.

However, an issue that emerged and remains unaddressed is the role the characteristics of the surrounding experimental facility might play in determining the pressures generated by the instability. More generally, it remains unknown what role these characteristics might play in initiating, sustaining and determining the frequency of the instability. Yet to be determined is the extent to which the instability is driven by intrinsic cavitation dynamics, and therefore might also occur under more open conditions, and to what extent the instability is characteristic of the particular facility in which the experiments are conducted.

\subsection{Modeling of the Facility Dynamics}

\subsubsection{System Impedance}

To better understand the role of the experimental facility dynamics, a model can be developed to predict the behavior of the facility in response to a volumetric excitation of frequency $\omega$, such as that provided by the changing cavity volume during the instability. 


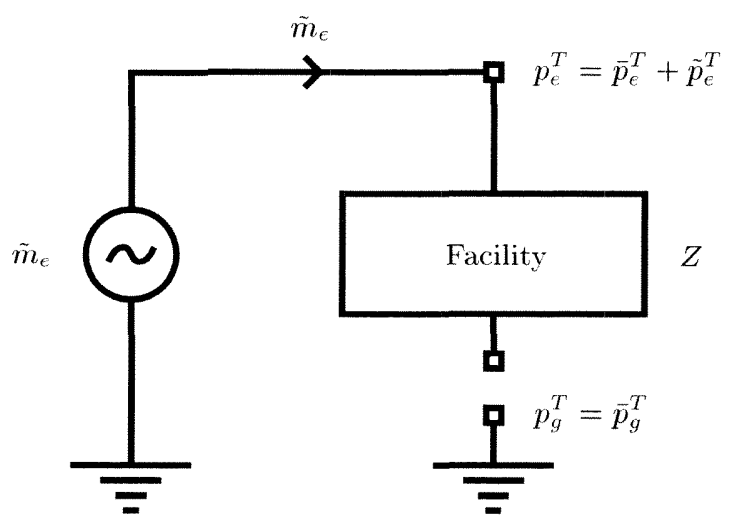

Figure 4.1: Schematic of facility excitation and impedance.

This situation is shown schematically in Figure 4.1, where a fluctuating mass flow rate, $\tilde{m}_{e}$, associated with the volumetric excitation is introduced into the facility and the resulting fluctuating total pressure at the point of excitation, $\tilde{p}_{e}^{T}$, is noted. From conservation of mass considerations, it is clear that the exact relation between the fluctuating volume, $\tilde{V}_{e}$, and the fluctuating mass flow rate is

$$
\tilde{m}_{e}=\rho \frac{d \tilde{V}_{e}}{d t} .
$$

In developing the model, the facility is assumed to respond in a linear manner to small perturbations in the flow conditions. Accordingly, the quantities of interest are expressed as a linear combination of a mean component and a fluctuating component of frequency $\omega$. The fluctuating mass flow rate, for example, is denoted by

$$
m_{e}=\bar{m}_{e}+\operatorname{Re}\left[\tilde{m}_{e} e^{j \omega t}\right]
$$

and the resulting fluctuating total pressure by

$$
p_{e}^{T}=\bar{p}_{e}^{T}+\operatorname{Re}\left[\tilde{p}_{e}^{T} e^{j \omega t}\right]
$$

Both $\tilde{m}_{e}$ and $\tilde{p}_{e}^{T}$ are complex and therefore incorporate both the amplitude and phase of the fluctuations. 
It is possible to define a system impedance, $Z$, relative to the constant pressure at ground, that fully characterizes the response of the facility to the excitation. This impedance is defined as

$$
Z=\frac{\tilde{p}_{e}^{T}-\tilde{p}_{g}^{T}}{\tilde{m}_{e}}=\frac{\tilde{p}_{e}^{T}}{\tilde{m}_{e}} .
$$

Like the fluctuating quantities, $Z$ is complex. Also, its value depends on the location of the excitation within the facility.

It is important to note that the pressures defined here are total pressures. Since the total pressure at a location is related to the static pressure by the relationship $p^{T}=$ $p+\frac{1}{2} \rho \bar{u}^{2}$, where $\bar{u}$ is the unperturbed, mean flow velocity,

$$
\tilde{p}^{T}=\tilde{p}+\rho \bar{u} \tilde{u}
$$

where variations in fluid density are assumed negligible. Thus, if no mean flow is present, the fluctuating total pressure and fluctuating static pressure may be used interchangeably, and

$$
Z=\frac{\tilde{p}_{e}^{T}}{\tilde{m}_{e}}=\frac{\tilde{p}_{e}}{\tilde{m}_{e}}
$$

\subsubsection{Lumped Parameters}

In developing a specific model for $Z$, one very convenient approach is to employ a lumped parameter analysis, dividing the experimental facility into components for which expressions can be written relating the fluctuating quantities at the inlet and outlet of the component. Figure 4.2 illustrates these relationships for a straight section of the facility and an accumulator.

For the straight section of the facility, conservation of mass clearly requires that the fluctuating mass flow rates be the same at inlet and outlet. However, a pressure drop will be present across the length of the section. First, there is a pressure drop, proportional to the fluctuating mass flow rate, associated with the frictional losses in the pipe. The constant of proportionality, termed the resistance of the straight section, is given by 


$$
\begin{gathered}
\tilde{p}_{2}^{T}=\tilde{p}_{1}^{T}-R \tilde{m}_{1}-j \omega L \tilde{m}_{1} \\
\tilde{m}_{2}=\tilde{m}_{1}
\end{gathered}
$$

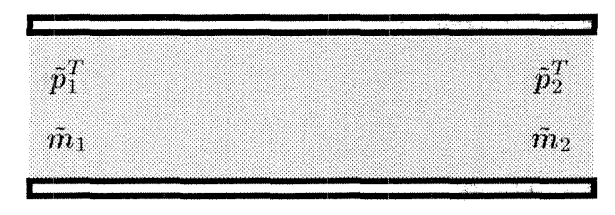

$$
\begin{gathered}
\\
\tilde{p}_{2}=\tilde{p}_{1} \\
\tilde{m}_{2}=\tilde{m}_{1}-j \omega C \tilde{p}_{1}
\end{gathered}
$$

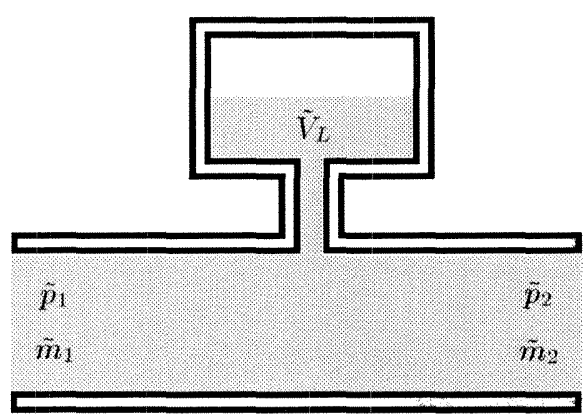

Figure 4.2: Lumped parameters utilized in experimental facility model. The equations at left show the relationships between inlet and outlet fluctuating quantities for a straight facility section (top) and an accumulator (bottom).

$R=\frac{f \bar{u} l}{A D}$, where $f$ is the friction factor, $\bar{u}$ the mean flow velocity, $l$ the length, $D$ the diameter, and $A$ the cross-sectional area of the straight section. In addition, there is a pressure drop proportional to the acceleration of the flow. The constant of proportionality, termed the inertance of the straight section, can be shown to have the value $L=\frac{l}{A}$, where $l$ is the length of the section and $A$ the cross-sectional area.

Another frequently encountered lumped parameter characteristic is the compliance. Generally speaking, a compliance occurs anywhere in the facility where mass can be periodically stored as a result of changing local pressure. A familiar example is the accumulator, or overflow tank, shown in Figure 4.2. In this case, although the fluctuating static pressures are equal at inlet and outlet, there is a change in the fluctuating mass flow rate due to the fact that changes in the inlet pressure will lead to a rise of the free surface of the liquid in the accumulator. The resulting mass stored in the accumulator is proportional to the change in fluctuating static pressure, according to the compliance $C=\rho \frac{d \tilde{V}_{L}}{d \tilde{p}_{1}}$. 


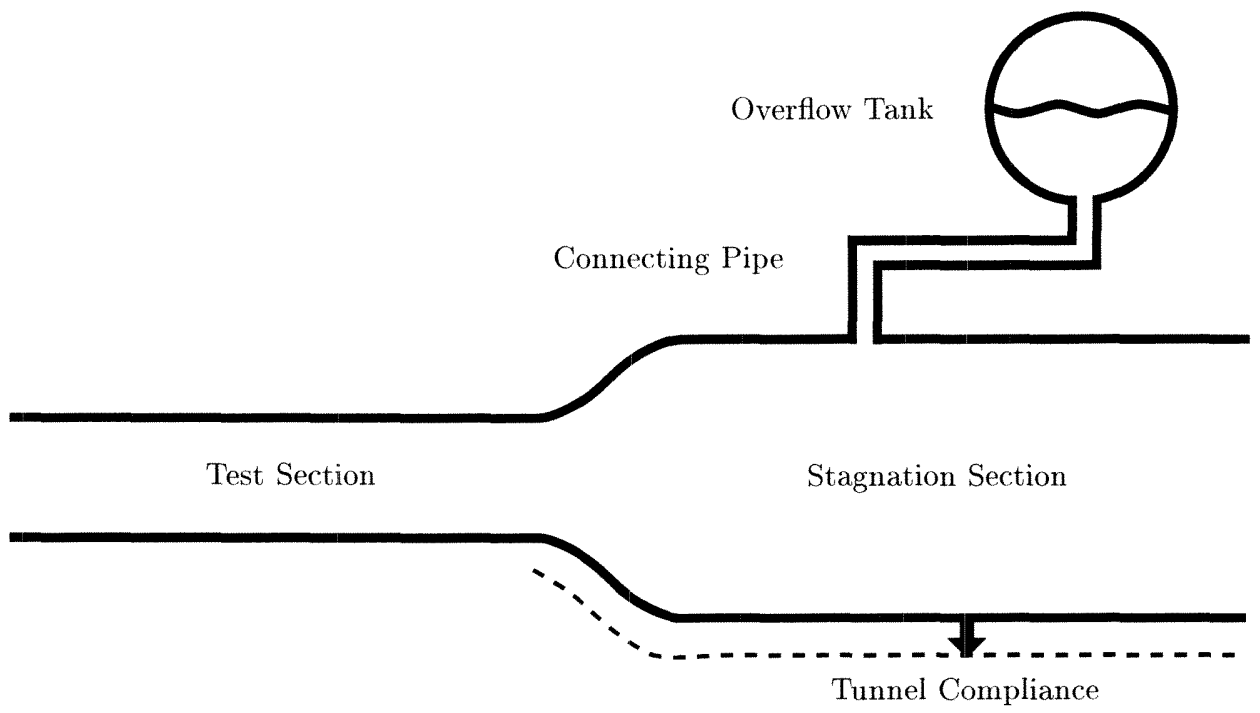

Figure 4.3: Significant features of experimental facility. Shown are the locations of the resistive, inertive, and compliant behaviors of the experimental facility.

\subsubsection{Characterization of the Experimental Facility}

These lumped parameter characteristics can then be used to model the dynamics of the experimental facility. Figure 4.3 shows an overview of a portion of the LTWT facility. Clearly, the overflow tank, which contains the free surface of the facility, will exhibit a compliant behavior akin to that of the accumulator shown in Figure 4.2. Also, the connecting pipe leading to the overflow tank from the stagnation section will exhibit resistive and inertive behaviors. Somewhat less obvious but equally important is the fact that the facility walls themselves will exhibit a compliant behavior, expanding outward and contracting inward in response to changing internal pressures. In addition to this tunnel compliance there will clearly be a tunnel resistance and a tunnel inertance, representative of the resistance and inertance along the typical flow path leading from the excitation to the tunnel compliance. This is a considerable simplification of the real system, where the tunnel compliance is actually distributed along the entire exterior of the facility, and therefore resides at the end of not one but infinitely many possible flow paths, each with a different resistance and inertance. In this regard, the system would be more accurately modeled with a distributed parameter approach. For simplicity, the lumped parameter 


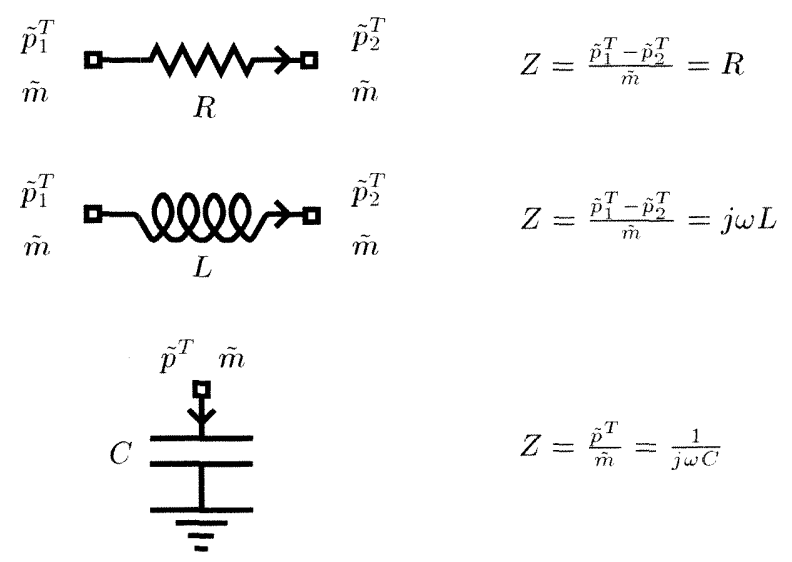

Figure 4.4: Impedance of lumped parameters. Schematic representations of resistive, inertive, and compliant components are presented, along with the associated impedances. analysis is used here.

By considering the nature and location of the compliances within the experimental facility, some important simplifications can be made. Since the overflow tank compliance is located at the end of a branch leading away from the main flow loop, there can be no mean flow leading to or from the overflow tank. As noted earlier, static and total pressures may then be used interchangeably. Although the tunnel compliance is distributed over the entire exterior of the facility, the great majority of this compliance is located at areas of large cross-section, and therefore at low mean and fluctuating flow velocities. From Equation 4.5, it is clear that in this case as well, the static and total pressures are nearly equivalent. For simplicity then, the compliant behaviors within the facility can be expressed as

$$
\begin{aligned}
\tilde{p}_{2}^{T} & =\tilde{p}_{1}^{T} \\
\tilde{m}_{2}^{T} & =\tilde{m}_{1}-j \omega C \tilde{p}_{1}^{T} .
\end{aligned}
$$

Having made these simplifications, it is possible to cast the behavior of each lumped parameter in the form of an impedance. Figure 4.4 shows a schematic representation of each type of parameter and the associated impedance. Using this notation, the dynamics of the water tunnel facility can described with the schematic representation shown in 


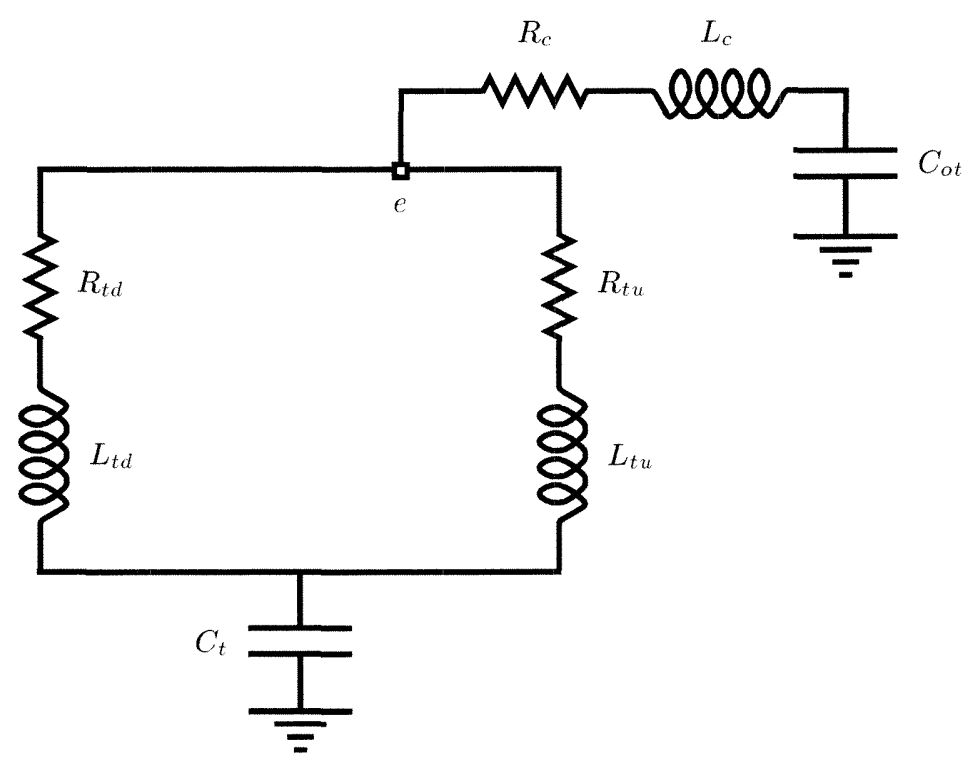

Figure 4.5: Schematic of experimental facility.

Figure 4.5. In this representation, $R_{c}$ and $L_{c}$ represent the resistance and inertance associated with the pipe connecting the stagnation section to the overflow tank, which is characterized by the compliance $C_{o t}$. Upstream of the point of excitation $e$ are the tunnel resistance and inertance $R_{t u}$ and $L_{t u}$ leading to the tumnel compliance, $C_{t}$. Likewise, the tunnel resistance and inertance $R_{t d}$ and $L_{t d}$ are located in the downstream direction. This network representation is particularly convenient in that the system impedances can be computed for various excitation locations utilizing the common rules for series and parallel combination of electrical circuit elements.

\subsubsection{Evaluation of the Lumped Parameters}

Several of the lumped parameters described in Section 4.1 .3 can be evaluated directly from measurements of the experimental facility. Table 4.1 itemizes these measurements. The overflow tank free surface area and the volume above the free surface are based on an overflow tank filled to the mid-line level.

The connecting pipe inertance, $L_{c}$, as indicated in Section 4.1.2, can be evaluated directly from the length and area of the connecting pipe. Assuming that the volume of air above the overflow tank is sealed and exhibits polytropic behavior, with $p_{o t} V_{o t}{ }^{k}=$ 


\begin{tabular}{||c|l|c||}
\hline Symbol & Quantity & Value \\
\hline \hline$l_{c}$ & Length of connecting pipe & $1.2 \mathrm{~m}$ \\
\hline$l_{t s}$ & Length of test section & $2.5 \mathrm{~m}$ \\
\hline$l_{d}$ & Length of diffuser section & $4.2 \mathrm{~m}$ \\
\hline$l_{s}$ & Length of stagnation section & $1.2 \mathrm{~m}$ \\
\hline$A_{c}$ & Area of connecting pipe & $2.0 \times 10^{-3} \mathrm{~m}^{2}$ \\
\hline$A_{t s}$ & Area of test section & $9.0 \times 10^{-2} \mathrm{~m}^{2}$ \\
\hline$A_{d}$ & Area of diffuser section & $2.1 \times 10^{-1} \mathrm{~m}^{2}$ \\
\hline$A_{s}$ & Area of stagnation section & $1.5 \mathrm{~m}^{2}$ \\
\hline$A_{o t}$ & Area of overflow tank & $6.3 \times 10^{-1} \mathrm{~m}^{2}$ \\
\hline$V_{o t}$ & Air volume of overflow tank & $1.5 \times 10^{-1} \mathrm{~m}^{3}$ \\
\hline
\end{tabular}

Table 4.1: Measurements of the experimental facility.

constant, the expression for a compliance of Section 4.1.2 can be explicitly evaluated for the geometry of the overflow tank. The resulting compliance is

$$
\begin{aligned}
C_{o t}{ }^{-1} & =\left[\frac{\bar{A}_{o t}}{g}\right]^{-1}+\left[\frac{\rho \bar{V}_{o t}}{\bar{p}_{o t} k}\right]^{-1} \\
C_{o t}{ }^{-1} & =C_{f s}{ }^{-1}+C_{s v}^{-1},
\end{aligned}
$$

where $\vec{A}_{o t}$ is the unperturbed area of the overflow tank free surface, $g$ the acceleration of gravity, $k$ the polytropic constant, and $\bar{V}_{o t}$ and $\bar{p}_{o t}$ the unperturbed volume and pressure of the air above the overflow tank free surface. It is evident from Equation 4.10 that the compliance of the overflow tank is composed of contributions from the rise and fall of the free surface itself and the compression and expansion of the sealed volume of gas above the free surface. Of course, the sealed volume compliance is relevant only when the overflow tank is in fact sealed. These results are evaluated numerically in Table 4.2 with the free surface at the overflow tank mid-line and the sealed volume of air at atmospheric pressure. 


\begin{tabular}{||c|l|c||}
\hline Symbol & Quantity & Value \\
\hline \hline$R_{c}$ & Connecting pipe resistance & $8 \mathrm{~m}^{-1} \mathrm{~s}^{-1}$ \\
\hline$L_{c}$ & Connecting pipe inertance & $6.02 \times 10^{2} \mathrm{~m}^{-1}$ \\
\hline$C_{f s}$ & Free surface compliance & $6.42 \times 10^{-2} \mathrm{~ms}^{2}$ \\
\hline$C_{s v}$ & Sealed volume compliance & $1.05 \times 10^{-3} \mathrm{~ms}^{2}$ \\
\hline$C_{o t}$ & Overflow tank compliance & $1.03 \times 10^{-3} \mathrm{~ms}^{2}$ \\
\hline$R_{t}$ & Tunnel resistance & $17 \mathrm{~m}^{-1} \mathrm{~s}^{-1}$ \\
\hline$L_{t u}$ & Upstream tunnel inertance & $15 \mathrm{~m}^{-1}$ \\
\hline$L_{t d}$ & Downstream tunnel inertance & $34 \mathrm{~m}^{-1}$ \\
\hline$C_{t}$ & Tunnel compliance & $7.88 \times 10^{-4} \mathrm{~ms}^{2}$ \\
\hline
\end{tabular}

Table 4.2: Numerical estimates of the lumped parameters.

Evaluating the resistance of the connecting pipe is somewhat more difficult. According to the linearized resistance presented in Section 4.1.2, the lack of a mean flow in the connecting pipe will result in zero resistance. However, it is possible to estimate a resistance for the purely oscillatory flow by assuming that the flow is a laminar Poiseuille flow, where the mass flow rate and pressure drop are then related (Munson, Young, and Okiishi 1994) by

$$
R_{c}=\frac{\Delta \tilde{p}}{\tilde{m}}=\frac{8 \pi \mu l_{c}}{\rho A_{c}{ }^{2}}
$$

yielding the numerical value shown in Table 4.2 .

The validity of this resistance is difficult to assess. The Reynolds number for the flow will of course depend on the exact mass flow rate entering the connecting pipe and will fluctuate about a mean value of zero. If, for example, the entire mass flow rate associated with the fluctuating cavity volume enters the connecting pipe at a frequency of $f=10 \mathrm{~Hz}$, 
the resulting amplitude of the fluctuating Reynolds number is

$$
|\operatorname{Re}|=\frac{\rho\left|\tilde{u}_{c}\right| D_{c}}{\mu}=\frac{\rho \omega\left|\tilde{V}_{e}\right| D_{c}}{\mu A_{c}}=5.6 \times 10^{4}
$$

This is well above transition to turbulence for steady, fully developed flow. However, as will be demonstrated later, the mass flow rate entering the connecting pipe is in fact a function of the excitation frequency. More often than not, only a small fraction of the mass flow rate associated with the fluctuating cavity volume enters the connecting pipe, and this high value of Reynolds number will not be attained. Furthermore, the peak amplitude of the Reynolds number is only attained when the fluctuating velocity in the pipe reaches a maximum. In general, the Reynolds number fluctuates between zero and this peak value. Finally, it should be noted that the assumption of Poiseuille flow is valid only for a fully developed flow. It is uncertain how this concept should be applied to an oscillating flow such as the one present in the pipe connecting the test section to the overflow tank.

Nonetheless, the value of $R_{c}$ in Table 4.2 does offer some indication that the magnitude of the connecting pipe resistive term is very small compared to the inertive and compliant terms of the connecting pipe and overflow tank behavior. Thus, the resistance would be significant near a resonance, where the inertive and compliant terms would be equal in magnitude but of opposite sign.

Accurately estimating the resistance of the tunnel, such as that described by $R_{t u}$ or $R_{t d}$ in Figure 4.5, is also challenging. In the absence of a mean flow, Equation 4.11 applies to the tunnel resistance as well. Due to the $\frac{1}{A^{2}}$ dependence of this expression, the tunnel resistance, located in areas of the facility with much greater cross-sectional area than the connecting pipe, is even less significant than the connecting pipe resistance. In the presence of a mean flow, the expression given in Section 4.1.2 would be valid. The difficulty in this case is choosing appropriate values for $\bar{u}_{t}, l_{t}, A_{t}$, and $D_{t}$ given the distributed nature of the tunnel compliance and the varying geometry of the tunnel cross-section. At the very least, any estimate of this parameter will depend on the exact location of the excitation. 
Like the connecting pipe resistance, though, this term is typically small in comparison with the inertive and compliant behaviors. Assuming that most of the tunnel resistance occurs in the test section, where $\bar{u}_{t}$ is large and $A_{t}$ and $D_{t}$ are small, the resistance can be estimated as $R_{t}=17 \mathrm{~m}^{-1} \mathrm{~s}^{-1}$. Here, values of $\bar{u}_{t}=3.75 \mathrm{~ms}^{-1}, l_{t}=5 \mathrm{~m}, A_{t}=0.09 \mathrm{~m}^{2}$, $D_{t}=0.3 \mathrm{~m}$, and $f=0.025$, as estimated from a Moody chart, have been utilized to arrive at this number, which is indeed small when compared to the inertive and compliant behaviors shown in Table 4.2 .

Attempts to evaluate the inertance of the tunnel, such as that of $L_{t u}$ or $L_{t d}$, are subject to the same difficulties as those encountered in evaluating the tunnel resistance. That is, determining a length and cross-sectional area characteristic of the flow path between the excitation and the compliance is very difficult. Also like the tunnel resistance, the value of the tunnel inertance will depend on the location of the excitation relative to the location of tunnel compliance. If the excitation is taken to be in the middle of the test section, the typical upstream flow path between excitation and compliance is taken to be composed of a test section half-length and the diffuser length, and the typical downstream flow path is taken to be composed of a test section half-length and the stagnation section length, then the tunnel inertances can be estimated as

$$
\begin{aligned}
L_{t u} & =\frac{l_{t s}}{2 A_{t s}}+\frac{l_{s}}{A_{s}} \\
L_{t d} & =\frac{l_{t s}}{2 A_{t s}}+\frac{l_{d}}{A_{d}} .
\end{aligned}
$$

Substituting numerical values for $l_{t s}, l_{s}, l_{d}, A_{t s}, A_{s}$, and $A_{d}$ yields the estimates shown in Table 4.2 .

The remaining parameter, the tunnel compliance associated with the expansion and contraction of the tunnel walls, can be estimated by directly measuring the response of the tunnel walls to changes in internal pressure. Figure 4.6 shows the results from such an experiment, where the tunnel pressure was varied and the resulting inward deflection of the tunnel walls was recorded at various locations on the exterior.

As shown in Figure 4.7, four of these locations were located on the approximately rectangular test section panel, which has a length of $126 \mathrm{~cm}$ and a height of $32 \mathrm{~cm}$. One 

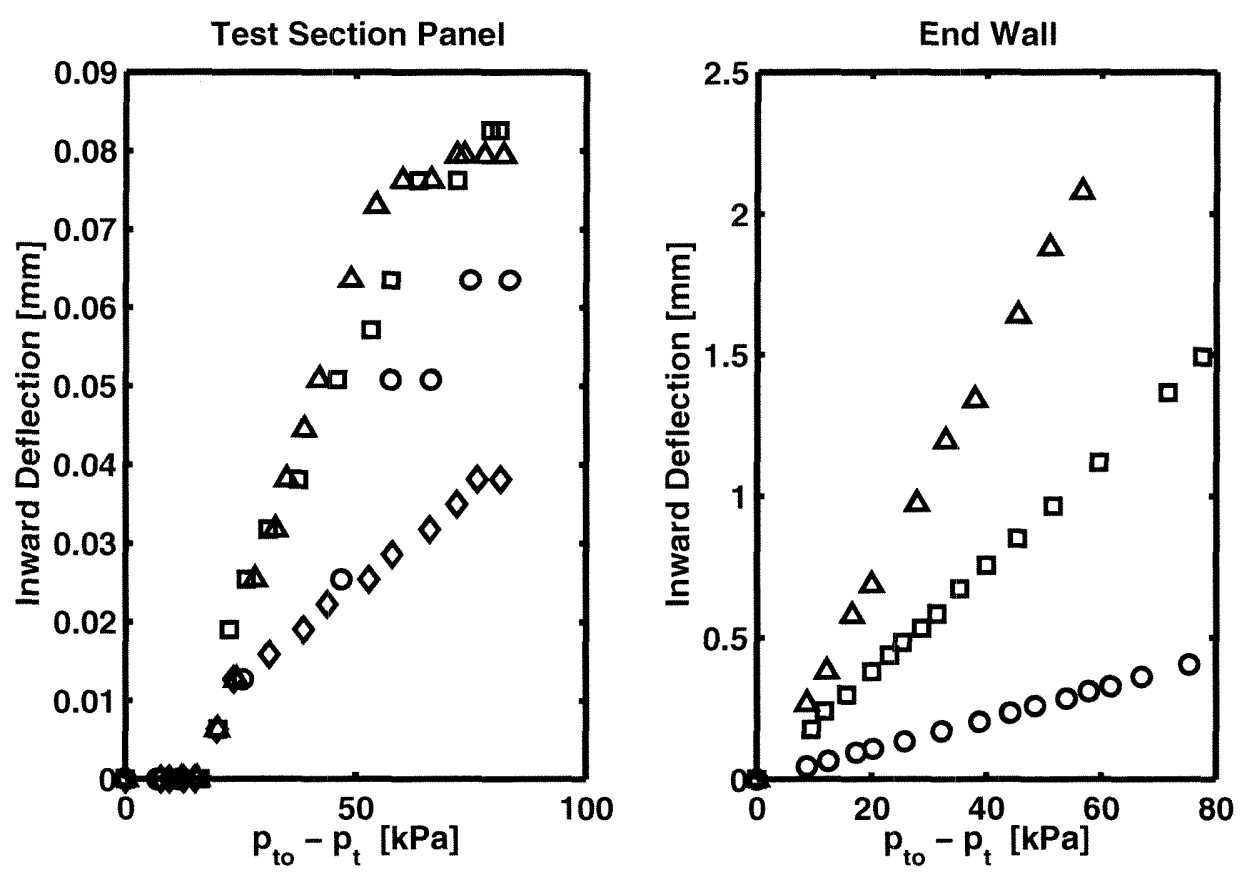

Figure 4.6: Experimentally measured deflection of tunnel walls. Presented is the inward deflection of the tunnel walls in response to a reduction of tunnel pressure, $p_{t}$, below an initial tunnel pressure, $p_{t o}$, at the locations $(\triangle, \square, \bigcirc, \diamond)$ defined in Figure 4.7.

set of measurements was obtained precisely at the center of this front panel, one set each at the midpoints of the short and long edges of the test section panel, $2.5 \mathrm{~cm}$ from the surrounding frame, and one set at the corner of the test section panel, $2.5 \mathrm{~cm}$ from both edges of the panel. A set of measurements was also taken at the center of the panel located on the backside of the test section, but the measurements at this point showed no substantial difference from those taken at the center of the front panel. Three additional sets of measurements were taken on a large metal end wall located downstream of the test section, immediately after the turning vanes direct the flow downward towards the flow return path. The three measurement sets were obtained at distances of $8 \mathrm{~cm}, 22 \mathrm{~cm}$, and $47 \mathrm{~cm}$ away from an essentially rigid supporting flange.

Figure 4.6 clearly shows that the amount of deflection varies greatly with location. Not surprisingly, the test section panel, which is more rigidly supported by its surrounding frame and constructed of thick glass, shows less deflection than the weaker end wall. Also as expected, the measurements taken at locations further from surrounding supports 


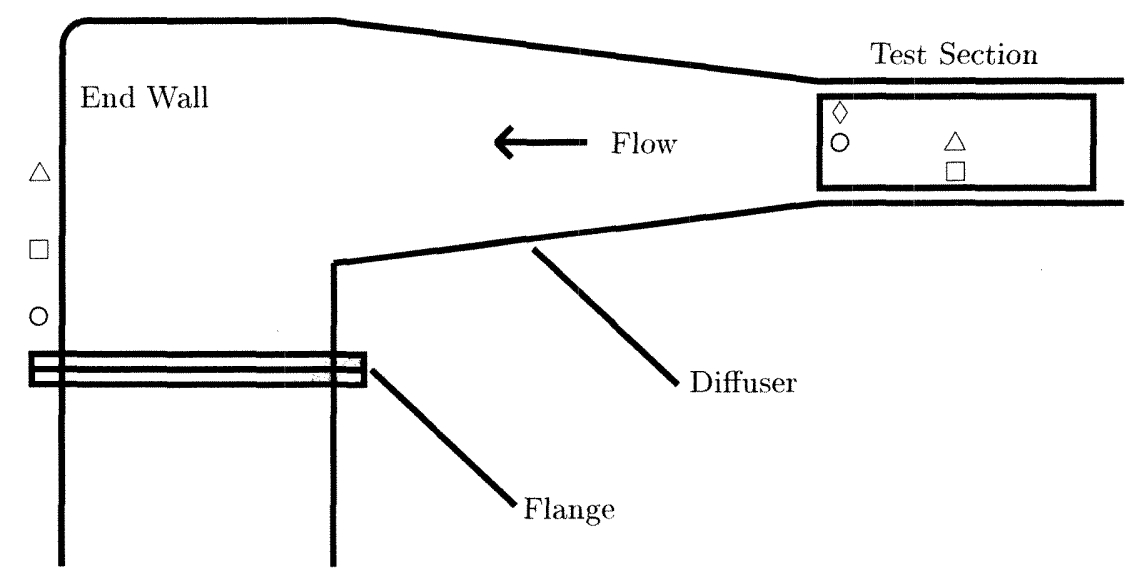

Figure 4.7: Location of tunnel wall deflection measurements. Deflection measurements were taken at the test section panel center $(\triangle)$, long edge midpoint $(\square)$, short edge midpoint $(\circ)$, and corner $(\diamond)$. Additional measurements were taken on the tunnel end wall at increasing distances $(O, \square, \triangle)$ from a nearby supporting flange.

exhibit greater deflection. Exactly why some locations on the test section panel exhibit a delay in the onset of expansion is not understood.

It is probable that other locations around the tunnel exterior, where material, and size, shape, and extent of reinforcement vary widely, would also show similar variation. Thus, it is possible to estimate from Figure 4.6 a range of values for the tunnel compliance. Returning to the definition of the compliance given in Section 4.1.2,

$$
C_{t} \doteq \rho \frac{d V_{t}}{d p_{t}}=\rho \frac{d V_{t}}{d s} \frac{d s}{d p_{t}}
$$

where $\frac{d s}{d p_{t}}$ is the slope of Figure 4.6 and $\frac{d V_{t}}{d s}$ is simply the surface area of the tunnel. Taking the tunnel surface area to be $75 \mathrm{~m}^{2}$, we can estimate that

$$
7.5 \times 10^{-5} m s^{2} \leq C_{t} \leq 1.5 \times 10^{-3} m s^{2} .
$$

A value in the middle of this range is recorded in Table 4.2 .

Finally, the compliance associated with the compressibility of the water within the 
tunnel can also be considered. This compliance is given by

$$
C_{L}=\frac{M_{t}}{E}
$$

where $E$ is the bulk modulus of water, and $M_{t}$ is the total mass of water within the facility. Noting that the total volume of the facility is $V_{t}=22.7 \mathrm{~m}^{3}$ (Gates 1977), and that the bulk modulus of water is $E_{V}=2.15 \times 10^{9} \mathrm{kgm}^{-1} \mathrm{~s}^{-1}$, this quantity can be estimated as $C_{L}=1.06 \times 10^{-5} \mathrm{~ms}^{2}$. Since this compliance combines additively with the tunnel compliance, it can be seen that the effects of water compressibility are rather insignificant, even in comparison to the smallest values of the tunnel compliance given in Equation 4.16.

In conclusion, due to the difficulties encountered in evaluating the connecting pipe resistance, tunnel resistance, tunnel inertance, and tunnel compliance, the values listed for $R_{c}, R_{t}, L_{t u}, L_{t d}$, and $C_{t}$ in Table 4.2 must only be considered rough estimates. The values of these parameters are better determined by considering the results from experiments exploring the dynamic behavior of the facility.

\subsection{Natural Frequency Experiments}

\subsubsection{Prediction and Measurement of the Natural Frequency}

According to Hennyey (1962), the natural frequency of a dynamic system such as the water tunnel facility is determined by the poles of the system impedance shown in Figure 4.1. More specifically, the natural frequency of the facility is given by the poles of the system depicted in Figure 4.5. The poles of this system are invariant with respect to the location of excitation and are consequently a system property, as would be expected for a natural frequency.

The poles of the system can therefore be evaluated by considering the impedance as calculated at an arbitrary excitation location. The location $e$ identified in Figure 4.5 is particularly convenient, in that the resulting impedance is readily identifiable as the parallel combination of the overflow tank and connecting pipe dynamics with the tunnel 
resistance, inertance and compliance. Computing the impedance for such an arrangement yields

$$
Z=\frac{j\left(L_{c} C_{o t} w^{2}-1\right)\left(L_{t} C_{t} w^{2}-1\right)}{w\left[\left(L_{c}+L_{t}\right) C_{o t} C_{t} w^{2}-C_{e q}\right]}
$$

where two new parameters, $L_{t}^{-1}=L_{t u}{ }^{-1}+L_{t d}{ }^{-1}$ and $C_{e q}=C_{o t}+C_{t}$, have been introduced. The resistive behaviors of the facility have been neglected for simplicity. In accordance with the reasoning presented in Section 4.1.4, the resistive values play little role in determining the locations of the natural frequencies, but instead principally affect the magnitude of the response at these natural frequencies.

This transfer function $Z$ has non-negative poles at the origin and at

$$
\omega_{o}=\left[\frac{C_{e q}}{\left(L_{c}+L_{t}\right) C_{o t} C_{t}}\right]^{\frac{1}{2}} .
$$

The estimated values of the lumped parameters given in Table 4.2 indicate that $L_{c} \gg L_{t}$, and thus this natural frequency is very nearly approximated by

$$
\omega_{o}=\left[\frac{1}{L_{c}}\left(\frac{1}{C_{o t}}+\frac{1}{C_{t}}\right)\right]^{\frac{1}{2}} .
$$

Finally, recalling the nature of the overflow tank compliance described by Equations 4.9 and 4.10 , it is clear that the natural frequency is a function of the conditions of the overflow tank through its dependence on $C_{o t}$. Specifically,

$$
\begin{aligned}
& \omega_{o}=\left[\frac{1}{L_{c}}\left(\frac{1}{C_{f s}}+\frac{1}{C_{s v}}+\frac{1}{C_{t}}\right)\right]^{\frac{1}{2}} \\
& \omega_{o}=\left[\frac{1}{L_{c}}\left(\frac{g}{\bar{A}_{o t}}+\frac{\bar{p}_{o t} k}{\rho \bar{V}_{o t}}+\frac{1}{C_{t}}\right)\right]^{\frac{1}{2}} .
\end{aligned}
$$

Inspection of this equation and the values of the parameters in Table 4.2 reveals that the natural frequency is dominated by the tunnel compliance. Of secondary importance is the sealed volume compliance, and nearly insignificant is the compliance associated with the free surface of the overflow tank.

Experiments were conducted to measure this natural frequency at various values of 


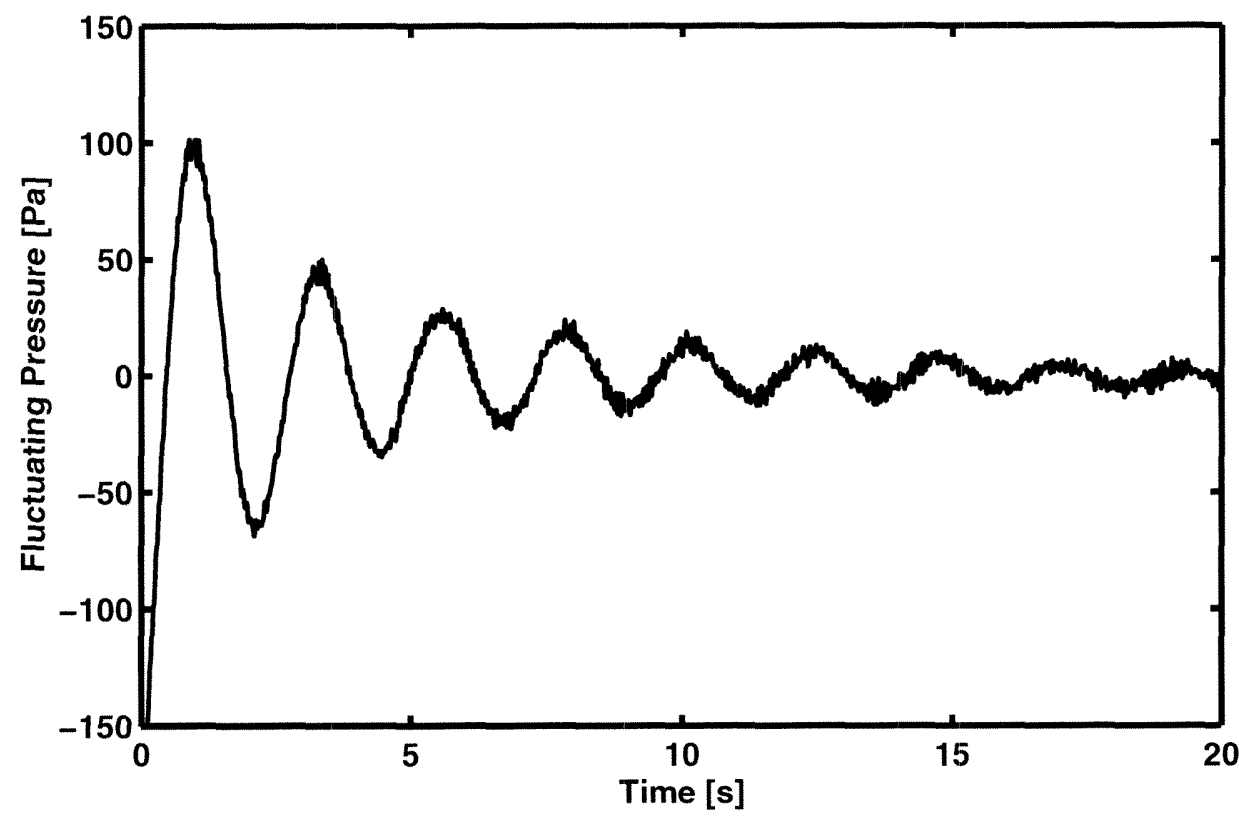

Figure 4.8: Fluctuating pressure during natural frequency experiments. The pressure fluctuations shown were obtained following a rapid valve closure at approximately $t=0 \mathrm{~s}$. The signal has been low-pass filtered at $20 \mathrm{~Hz}$.

the parameters determining the frequency in Equation 4.22. In these experiments, the tunnel pressure was perturbed by a rapid valve closure and then allowed to oscillate freely. Shown in Figure 4.8 is a typical pressure trace following the valve closure, as measured by the pressure transducer mounted at the test section centerline. The frequency of this oscillation, the natural frequency of the facility, was determined using a correlation method applied to the steady oscillations following the valve closure transients. Valves at various locations around the facility were employed in inducing the oscillations, with no noticeable effect on the frequency of oscillation. This supports the notion that the oscillations induced in this manner do characterize the natural frequency of the system, a property invariant with respect to the location of excitation.

\subsubsection{Effect of Overflow Tank Conditions}

A preliminary set of experiments was conducted to confirm the relative unimportance of the compliance of the sealed volume in the overflow tank, $C_{s v}$. In these experiments, the natural frequency was measured with the overflow tank closed and then opened to 
atmosphere, in an attempt to include and then exclude the sealed volume compliance. With the overflow tank closed, a natural frequency of $f=0.43 \pm 0.01 \mathrm{~Hz}$ was obtained. With the overflow tank opened to atmosphere, the frequency decreased slightly to $f=$ $0.41 \pm 0.01 \mathrm{~Hz}$. These figures are based on an average of five measurements at each overflow tank condition.

From this data, an immediate refinement for the value of the tunnel compliance given in Equation 4.16 can be obtained. Matching the natural frequency determined for the closed overflow tank to the predictions of Equation 4.22, including the sealed volume compliance, yields a value of $C_{t}=2.9 \times 10^{-4} \mathrm{~ms}^{2}$. Excluding the sealed volume compliance then results in a change in natural frequency of $\Delta \omega_{o}=0.05 \mathrm{~Hz}$, which is generally consistent with the $0.02 \mathrm{~Hz}$ difference obtained from the experiments. It is possible that the smaller decrease in natural frequency observed experimentally is due to the fact that the relief valve connecting the overflow tank to atmosphere may not have been large enough to fully eliminate the effects of the sealed volume compliance. In either case, these results confirm that the effect of the sealed volume compliance on the natural frequency is small.

Figure 4.9 shows the effect on the natural frequency of varying the overflow tank free surface area, $\bar{A}_{o t}$, and includes a comparison with the predictions of Equation 4.22. The free surface area was varied by controlling the height of the free surface within the horizontally mounted cylindrical overflow tank. Unfortunately, due to the relatively imprecise mechanism through which the free surface height was controlled, only a narrow range of free surface areas could be explored without risking overflow of the overflow tank.

Because these experiments were conducted with the overflow tank opened to atmosphere, the sealed volume compliance, $C_{s v}$, was not included in calculating the overflow tank compliance in Equation 4.22. Due to the extremely weak dependence of the natural frequency on the free surface compliance, and therefore the free surface area, the natural frequency should show very little variation with changing free surface area. Instead, it should be dominated by the value selected for the tunnel compliance. Indeed this is the case, as shown by the curves in Figure 4.9, generated from Equation 4.22 for several different tunnel compliance values. As noted earlier, the curve generated using the tunnel compliance value determined from the closed overflow tank experiments, 


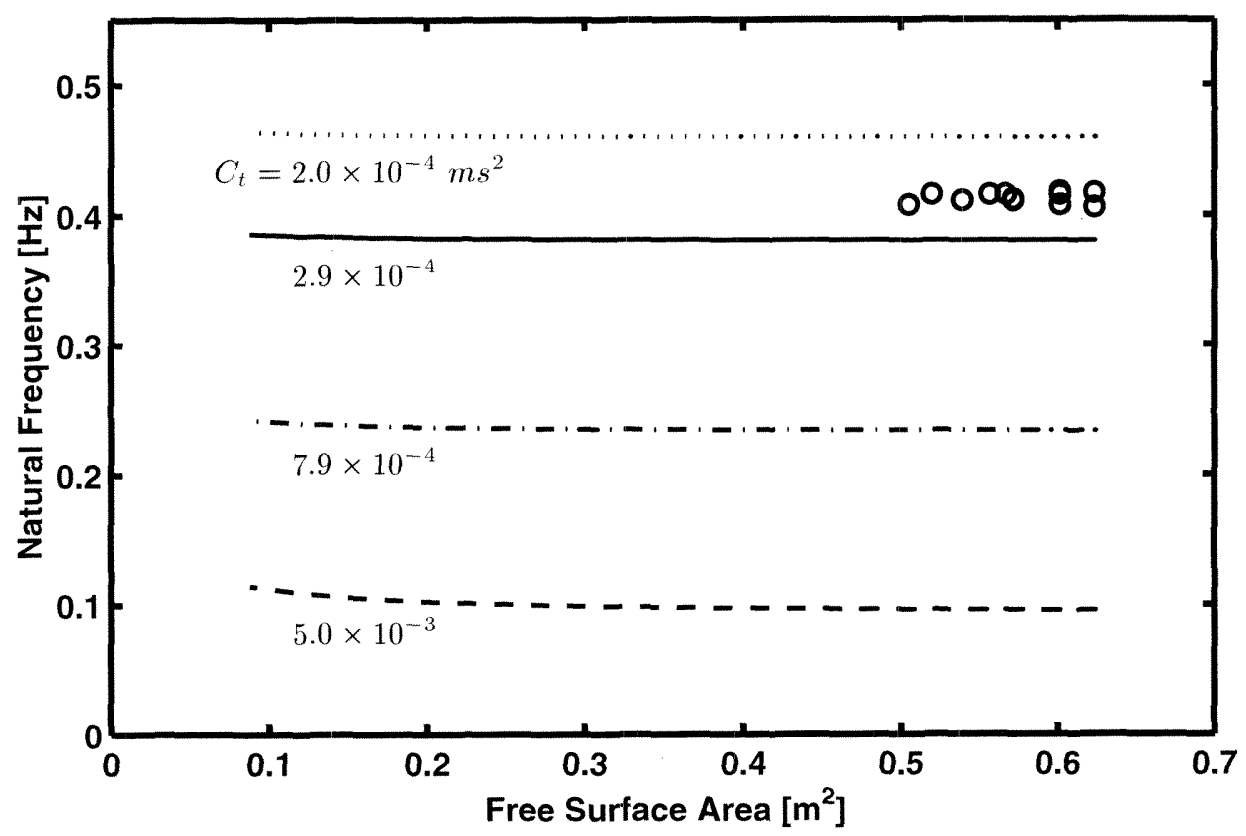

Figure 4.9: Natural frequency with changing overflow tank free surface area. Experimental data (O) and predictions of Equation 4.22 for several values of the tunnel compliance, including those based on the closed overflow tank experiments (-) and Table $4.2(\cdot-\cdot)$.

$C_{t}=2.9 \times 10^{-4} \mathrm{~ms}^{2}$, slightly under predicts the natural frequency observed experimentally for the open overflow tank.

Shown in Figure 4.10 are the experimentally determined natural frequencies for various pressures, $\bar{p}_{\text {ot }}$, above the free surface in the overflow tank. The predictions of Equation 4.22 are again shown, for the same values of $C_{t}$ as in Figure 4.9. As these experiments were conducted with the overflow tank closed to atmosphere, the effect of the sealed volume compliance is included in this case. As expected, the curve corresponding to the tunnel compliance value determined from the initial closed overflow tank experiments, $C_{t}=$ $2.9 \times 10^{-4} \mathrm{~ms}^{2}$, agrees very well with the experimentally determined natural frequencies at higher overflow tank pressures. It is also apparent that the curves generated for larger tunnel compliance values exhibit more variation with changing overflow tank pressure. This is due to the fact that for larger values of $C_{t}$, the effect of varying $C_{s v}$ is more significant. 


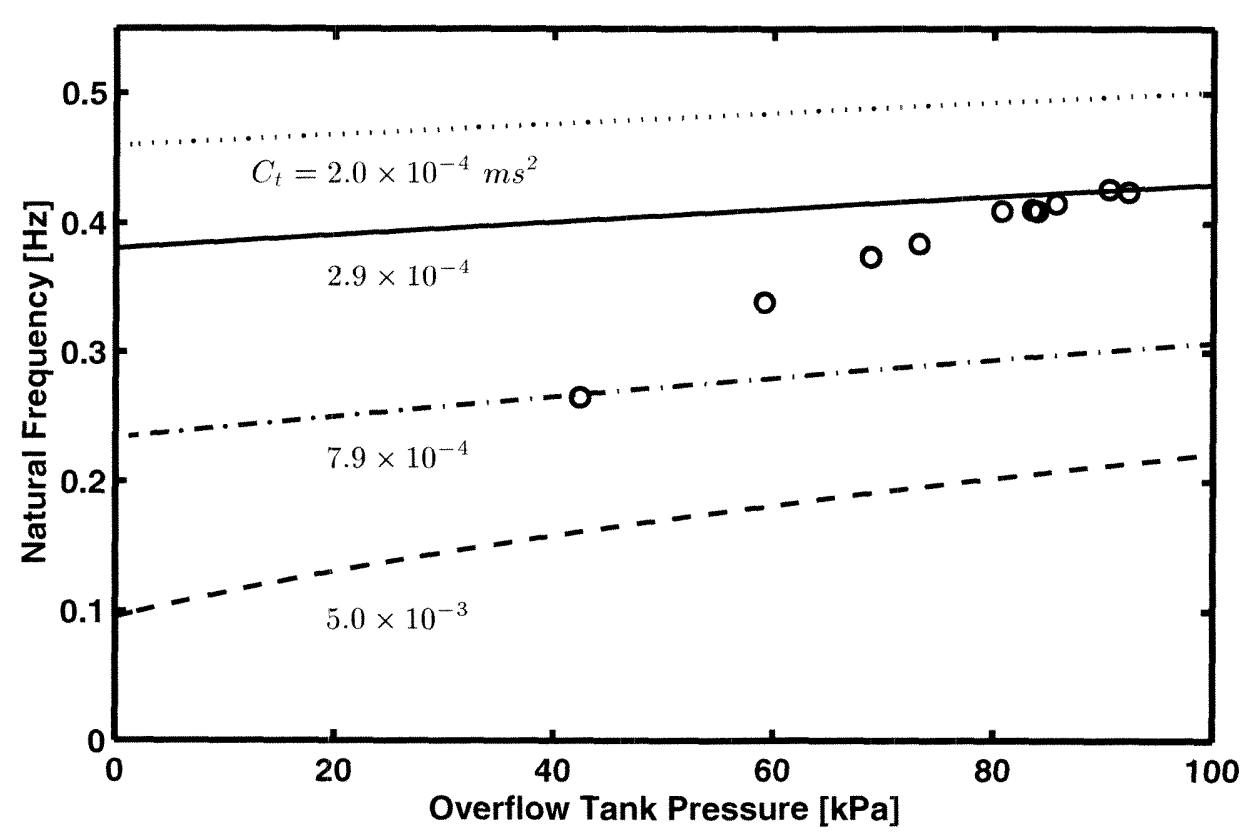

Figure 4.10: Natural frequency with changing overflow tank pressure. Experimental data (o) and predictions of Equation 4.22 for several values of the tunnel compliance, including those based on the closed overflow tank experiments (-) and Table $4.2(\cdot-\cdot)$.

\subsubsection{Effect of Air on Tunnel Compliance}

None of the curves in Figure 4.10 predicted by Equation 4.22 show the same degree of variation in natural frequency with changing overflow tank pressure as that exhibited by the experimental data. This suggests that the tunnel compliance, which dominates the natural frequency calculation, may not be constant with varying pressure as is suggested in Section 4.1.4.

One possible explanation for a tunnel compliance that varies with pressure is the presence of air within the facility, either as free bubbles dispersed throughout the facility or larger pockets of air trapped in discrete locations. In either case, the volume of air would serve to increase the tunnel compliance, resulting in a modified tunnel compliance dependent on the compliance of the air volume. Because this air would behave as a sealed volume with a compliance of $C=\frac{\rho \bar{V}}{\bar{p} k}$, the modified tunnel compliance would vary with the local pressure near the air and therefore with the overflow tank pressure.

A reasonable estimate for the volume of air associated with dispersed bubbles, $V_{d a}$, can be computed from the bubble populations determined for the facility by Liu (1995). 
Specifically,

$$
V_{d a}=\frac{4 \pi V_{t}}{3} \int_{0}^{\infty} N(R) R^{3} d R
$$

where $V_{t}$ is the total volume of water within the facility and $N(R)$ the nuclei number density distribution function, the number of free stream nuclei with radii between $R$ and $R+d R$ contained within a unit volume of water. Carrying out this calculation results in a total volume of $V_{d a}=3.5 \times 10^{-4} \mathrm{~m}^{3}$. This volume is very similar to the value of $V_{d a}=3.2 \times 10^{-4} \mathrm{~m}^{3}$ calculated by simply multiplying the total volume of water in the facility by a typical total air content of $14 \mathrm{ppm}$. The associated compliance, at atmospheric pressure, is then readily estimated as $2.4 \times 10^{-6} \mathrm{~ms}^{2}$. Clearly, this compliance would add little to the existing tunnel compliance calculated in Section 4.1.4.

Estimating the volume of the trapped air pockets is considerably more difficult. It is entirely possible, though, that this volume of air could easily exceed the estimate for $V_{d a}$. Accordingly, the effect of these trapped air pockets on the natural frequency is shown in Figure 4.11 for a range of trapped air volumes. In each case, the tunnel compliance is adjusted so that the resulting modified tunnel compliance yields the proper natural frequency with the overflow tank at atmospheric pressure. These calculations are compared with the experimental data, repeated from Figure 4.10. For a trapped air volume of $V_{t a}=17 \times 10^{-3} \mathrm{~m}^{3}$, the agreement between experiment and theory is greatly improved.

Further insight into the possible effects of trapped air can be gained by considering the effects of deaerating the facility on the natural frequency. Presumably, the deaeration process, conducted with the tunnel slowly flowing and mixing, would allow these pockets of air to be dissolved into the water and removed from the facility. Figure 4.12 shows experimentally determined natural frequencies before and after deaeration. The volume of trapped air before deaeration is taken to be the volume that offered the best agreement with the data in Figure 4.11, and the volume after deaeration is determined by a fit to the experimental data taken after deaeration. In this case, the tunnel compliance is held constant at the value used in fitting the data obtained before deaeration. 


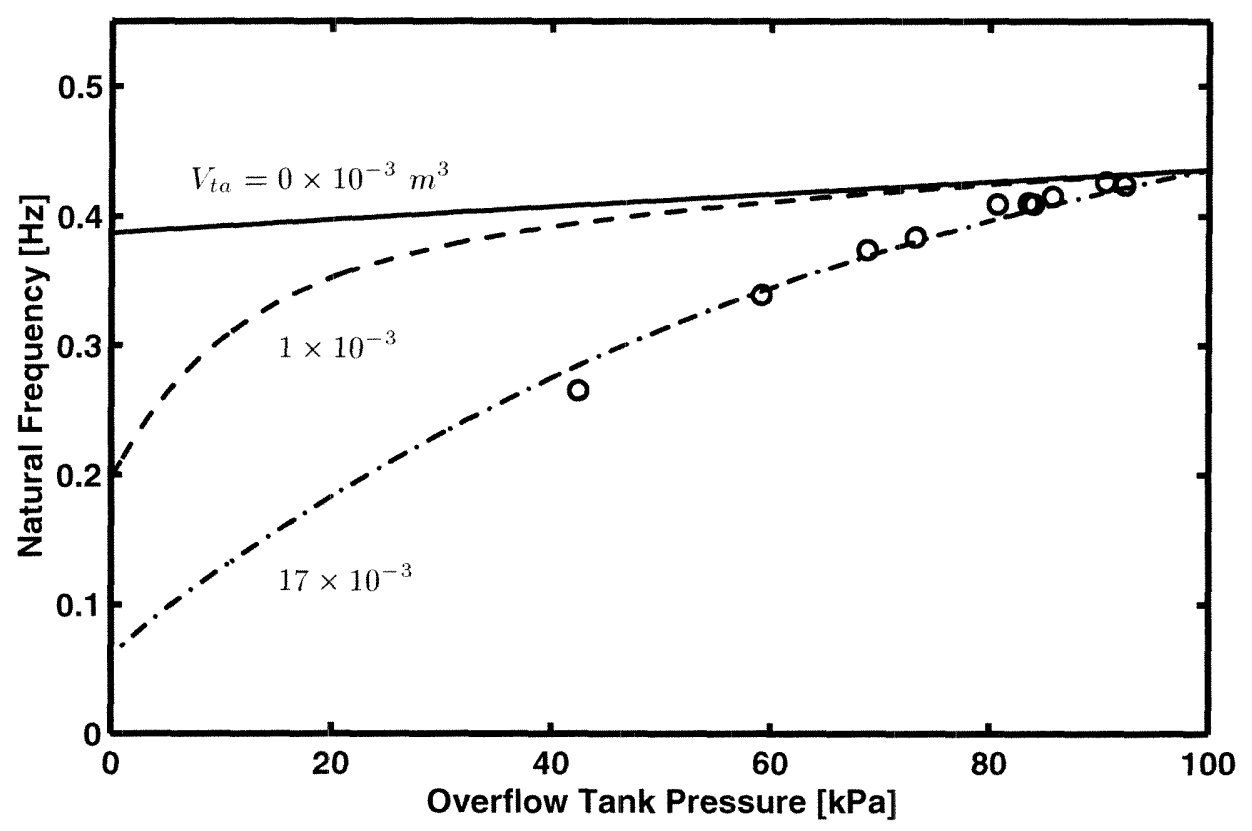

Figure 4.11: Effect of trapped air on natural frequency. The experimentally determined natural frequencies are shown (O), along with the natural frequency predicted by Equation 4.22, including the effects of trapped air on the tunnel compliance. Curves are shown for various volumes of trapped air.

Indeed, the volume of air offering the best fit to the after deaeration experiments, $V_{t a}=$ $7 \times 10^{-3} \mathrm{~m}^{3}$, is a reasonable estimate in comparison to the volume of $V_{t a}=17 \times 10^{-3} \mathrm{~m}^{3}$ hypothesized before deaeration. Still, it is impossible to conclude definitively whether or not trapped pockets of air are the actual source of the variations in natural frequency with overflow tank pressure. It remains certain, though, that the tunnel compliance is the dominant factor in determining the natural frequency of the facility. As such, it would seem appropriate to determine a specific value for $C_{t}$ within the range of Equation 4.16 by matching the predictions of Equation 4.22 to the experimental results of Figure 4.10 at the overflow tank pressure appropriate for the specific circumstances. 


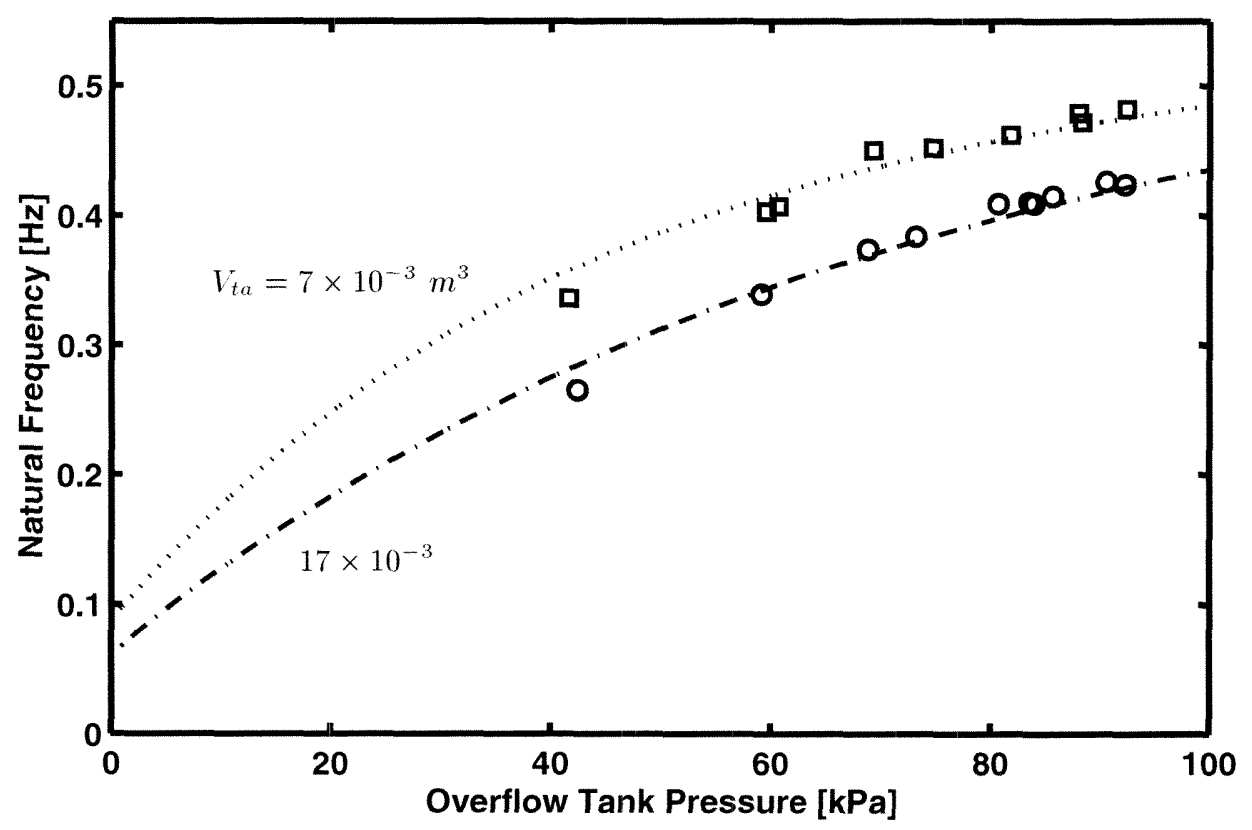

Figure 4.12: Effect of deaeration on natural frequency. The experimentally determined natural frequencies before $(0)$ and after $(\square)$ deaeration are shown, along with the natural frequency predicted by Equation 4.22, including the effects of the trapped air volumes before $(\cdot-\cdot)$ and after $(\cdots)$ deaeration.

\subsection{Forced Oscillation Experiments}

\subsubsection{Experimental Determination of the Facility Response}

More than just predicting the natural frequencies of the system, the system impedance described in Figure 4.1 and developed in Section 4.1.3 indicates how the system will respond to an excitation of arbitrary frequency. More can be learned about the parameters determining this behavior by considering experiments where a fluctuating mass flow rate is injected into the facility at various frequencies. Figure 4.13 shows an example of the pressure signals measured during such an experiment, utilizing the oscillating piston setup described in Section 2.3.1. It should be noted that the combination of piston diameter, $D_{p}=38 \mathrm{~mm}$, and range of piston oscillation amplitudes, $\left|\tilde{x}_{p}\right|=3.0-15.0 \mathrm{~mm}$, was chosen to approximate the change in cavity volume observed throughout the cavitation instability cycle. The range of oscillation frequencies explored, $f=0-20 \mathrm{~Hz}$, was sufficient to include the typical instability frequency of $f=10 \mathrm{~Hz}$. 


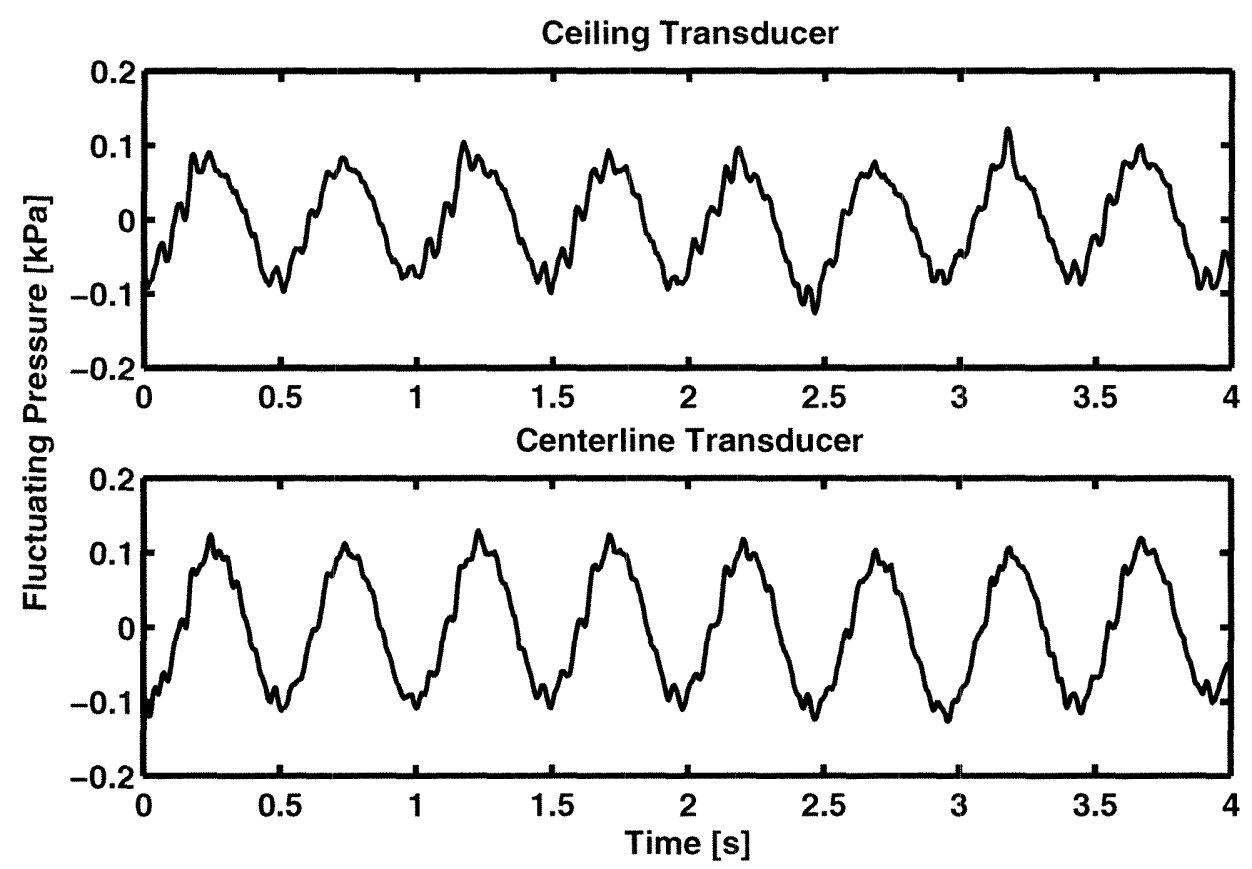

Figure 4.13: Fluctuating pressure during forced response experiments. Presented are typical pressure fluctuations recorded by the ceiling-mounted and test section mid-line pressure transducers during the oscillating piston experiments. Nominal excitation frequency is $f=2.0 \mathrm{~Hz}$, oscillation amplitude $\left|\tilde{x}_{p}\right|=15.7 \mathrm{~mm}$. The signal has been low-pass filtered at $25 \mathrm{~Hz}$.

The majority of these forced oscillation experiments were conducted with no mean flow present. Thus, the fluctuations in static pressure are identically equal to the fluctuations in total pressure, and the impedance defined in Equation 4.4 is directly applicable to the measured pressure fluctuations. For simplicity, it is appropriate to neglect the resistances and inertances located between the point of excitation and the location of the ceilingmounted transducer, $c$, and between $c$ and the point of connection to the overflow tank. Essentially, this renders the point of excitation and point $c$ as equivalent to point $e$ of Figure 4.5. The resulting system impedance is then the parallel configuration evaluated in Equation 4.18. If these resistances and inertances are considered, a bridge network configuration is achieved. Although the resulting system impedance is considerably more complex algebraically, the behavior is very similar to the simplified system addressed here.

Having made this assumption, the system impedance can be approximated from the 
experimental measurements according to the relation

$$
Z=\frac{\tilde{p}_{e}}{\tilde{m}_{e}}=\frac{\tilde{p}_{c}}{\tilde{m}_{e}}
$$

As implied by Equation 4.1, $\tilde{m}_{e}$ is related to the oscillating piston position, $\tilde{x}_{p}$, and the frequency of piston oscillation and is given by

$$
\tilde{m}_{e}=j \omega \rho A_{p} \tilde{x}_{p}
$$

where $A_{p}$ is the area of the piston bore.

\subsubsection{Magnitude of Response}

Figure 4.14 shows the amplitude of the system impedance, $|Z|$, for low frequencies and for several piston amplitudes, using pressure fluctuations measured by the ceiling-mounted transducer. Equation 4.18 is plotted for comparison. With the pressure of the facility adjusted to balance the pressures on either side of the piston, the resulting overflow tank pressure was $\bar{p}_{o t}=92.4 \mathrm{kPa}$. The overflow tank compliance used in Equation 4.18 has been adjusted accordingly, to a value of $C_{o t}=1.16 \times 10^{-3} \mathrm{~ms}^{2}$. For now, both resistances, $R_{c}$ and $R_{t}$, have been neglected.

Worth noting is that the data obtained at different piston amplitudes collapses quite well when normalized by the amplitude of the resulting fluctuating mass flow rate, especially at lower frequencies. This indicates that the facility response is in fact linear with the amplitude of excitation, as is assumed throughout the modeling.

Also apparent is that the peak in Figure 4.14 occurs at a slightly higher frequency, around $f=0.5 \mathrm{~Hz}$, than the natural frequency measured in Section 4.2.2. This may be attributable to the fact that between the natural frequency and forced oscillation experiments, the facility was drained and refilled. If, in fact, the effective tunnel compliance is dependent on the volume of trapped air within the facility as was speculated, the natural frequency may have thus been altered between these two experiments. Accordingly, in generating the curve of Equation 4.18, a slightly modified value of $C_{t}=2.40 \times 10^{-4} \mathrm{~ms}^{2}$ was utilized to best match the experimentally observed resonance. 


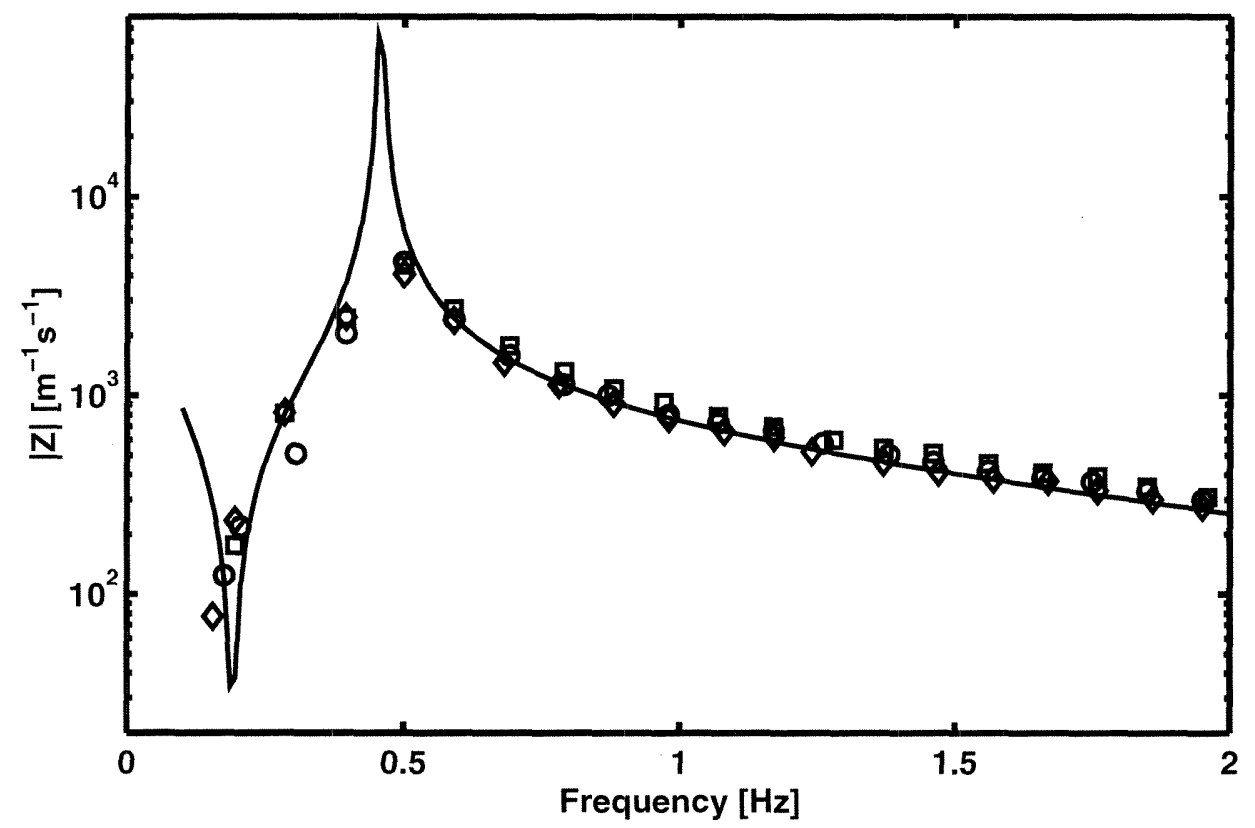

Figure 4.14: Magnitude of system impedance at low frequencies. $|Z|$, as determined experimentally for $\left|\tilde{x}_{p}\right|=6.5(0), 12.4(\diamond)$, and $15.7 \mathrm{~mm}(\square)$. Equation $4.18(-)$ is plotted for a value of $L_{t}=6.75 \mathrm{~m}^{-1}$, as determined in Figure 4.15 .

Easily distinguished in both the experimental data and the theoretical curve is the zero of the system impedance occurring at approximately $f=0.2 \mathrm{~Hz}$. This zero corresponds to the root of the numerator of Equation 4.18,

$$
\omega_{z}=\left[\frac{1}{L_{c} C_{o t}}\right]^{\frac{1}{2}}
$$

The response of the facility at the frequencies below resonance is thus dominated by the characteristics of the overflow tank. The excellent agreement between the theoretical curve and the experimental data near this zero supports the values of $L_{c}$ given in Table 4.2 and the adjusted value of $C_{o t}$ used in generating the theoretical curve.

Figure 4.15 shows the results of similar experiments and calculations performed at higher frequencies. At these higher frequencies, the data shows a substantial amount of scatter. This is partly attributable to the fact that operating at these higher frequencies approached the mechanical limits of the oscillating piston setup. The resulting piston motion was often not sinusoidal, with varying degrees of "banging" and "gallop- 


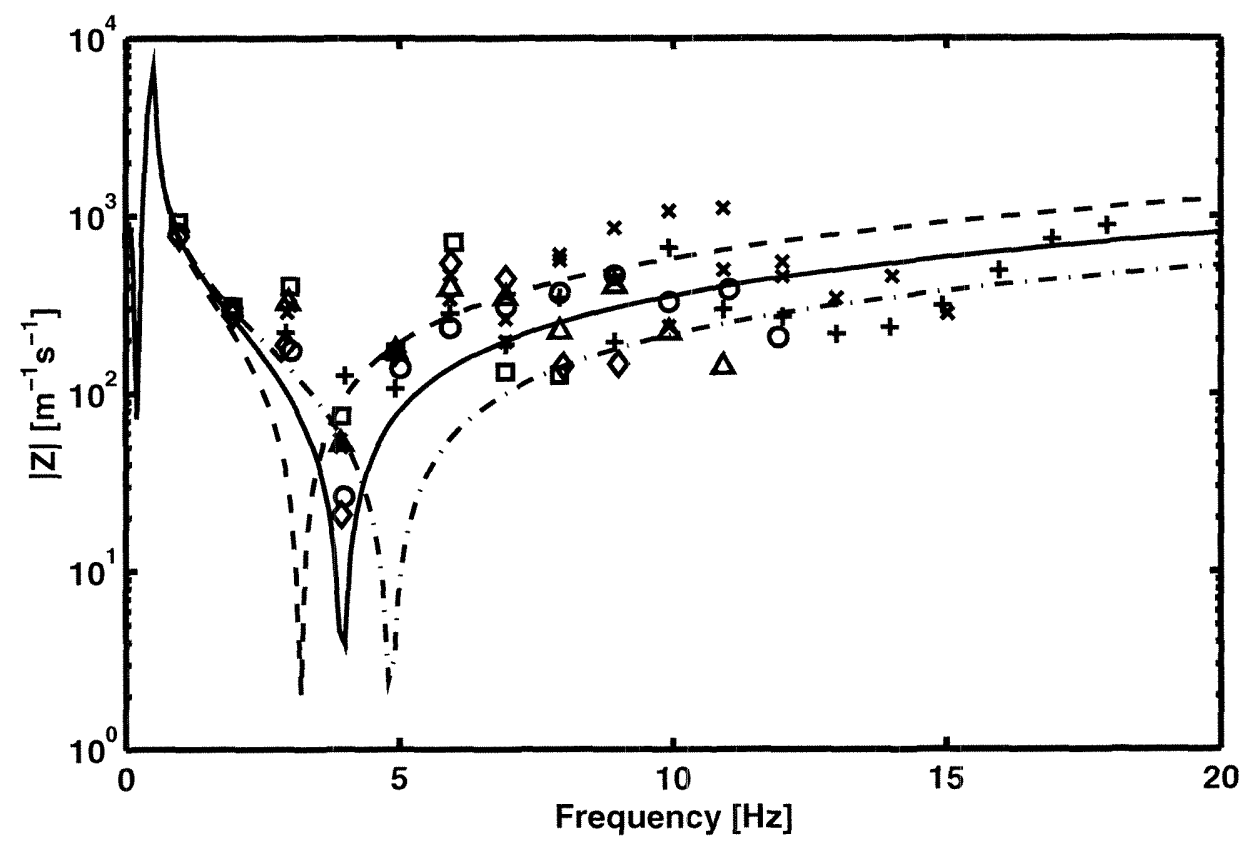

Figure 4.15: Magnitude of system impedance at high frequencies. $|Z|$, as determined experimentally for $\left|\tilde{x}_{p}\right|=1.6(+), 2.9(\times), 6.5(\circ), 9.0(\triangle), 12.4(\diamond)$, and $15.7 \mathrm{~mm}(\square)$. Equation 4.18 is plotted for values of $L_{t}=4.50(\cdot-\cdot), 6.75(-)$, and $10.4 \mathrm{~m}^{-1}(--)$, the value based on Table 4.2 .

ing" present. Also at higher frequencies, the pressure traces obtained from the transducers became increasingly more difficult to analyze, often exhibiting unsteady oscillations and sub-harmonics. At times, the amplitude of the sub-harmonic frequency component exceeded the amplitude of the excitation frequency component within the response. This suggests that the behavior of the piston excitation system becomes more non-linear with increasing frequency. In these cases, the amplitude of the excitation frequency component was used in determining the magnitude of the system response. Although this methodology is consistent with the linear model proposed for the facility, it does not account for the substantial energy present in the response at frequencies other than the excitation frequency. The larger piston amplitudes were especially problematic. Often, meaningful data was simply not attainable for the larger piston amplitudes at the highest frequencies.

For these reasons, it is not surprising to see the agreement between the predictions of Equation 4.18 and the experimental results degrade at higher frequencies. Nonetheless, readily apparent in both the experimental results and the curves generated from Equa- 


\begin{tabular}{||c|l|c||}
\hline Symbol & Quantity & Value \\
\hline \hline$R_{c}$ & Connecting pipe resistance & $60 \mathrm{~m}^{-1} \mathrm{~s}^{-1}$ \\
\hline$R_{t}$ & Tunnel resistance & $20 \mathrm{~m}^{-1} \mathrm{~s}^{-1}$ \\
\hline$L_{t u}$ & Upstream tunnel inertance & $10 \mathrm{~m}^{-1}$ \\
\hline$L_{t d}$ & Downstream tunnel inertance & $22 \mathrm{~m}^{-1}$ \\
\hline$C_{t}$ & Tunnel compliance & $2.65 \times 10^{-4} \mathrm{~ms}^{2}$ \\
\hline
\end{tabular}

Table 4.3: Revised estimates of the lumped parameters.

tion 4.18 is a second zero located at approximately $4.0 \mathrm{~Hz}$. This zero corresponds to the second root of the numerator of Equation 4.18,

$$
\omega_{z}=\left[\frac{1}{L_{t} C_{t}}\right]^{\frac{1}{2}}
$$

The behavior of the system at frequencies above resonance is thus dominated by the characteristics of the main tunnel circuit as opposed to those of the overflow tank. Because a reasonable value for the tunnel compliance was already determined from the natural frequency experiments and further refined for these particular experiments in Figure 4.14, this zero offers an excellent opportunity to better estimate the value of the tunnel inertance. Accordingly, the theoretical curves are plotted for several values of $L_{t}$ in Figure 4.15. A value of $L_{t}=6.75 \mathrm{~m}^{-1}$ provides the best fit to the experimental data.

Of course, the experimental data provides no indication of how this combined tunnel inertance is divided upstream and downstream of the point of excitation. Preserving the ratio between $L_{t u}$ and $L_{t d}$ of Table 4.2 yields the revised values shown in Table 4.3. Also shown is a revised value for the tunnel compliance based on the average of the natural frequency and forced response experiments.

\subsubsection{Phase of Response}

Figure 4.16 shows the experimentally determined phase of the facility response, $\operatorname{Arg}[Z]$, and that predicted by Equation 4.18. Due to the poor quality of the fluctuating pressure 


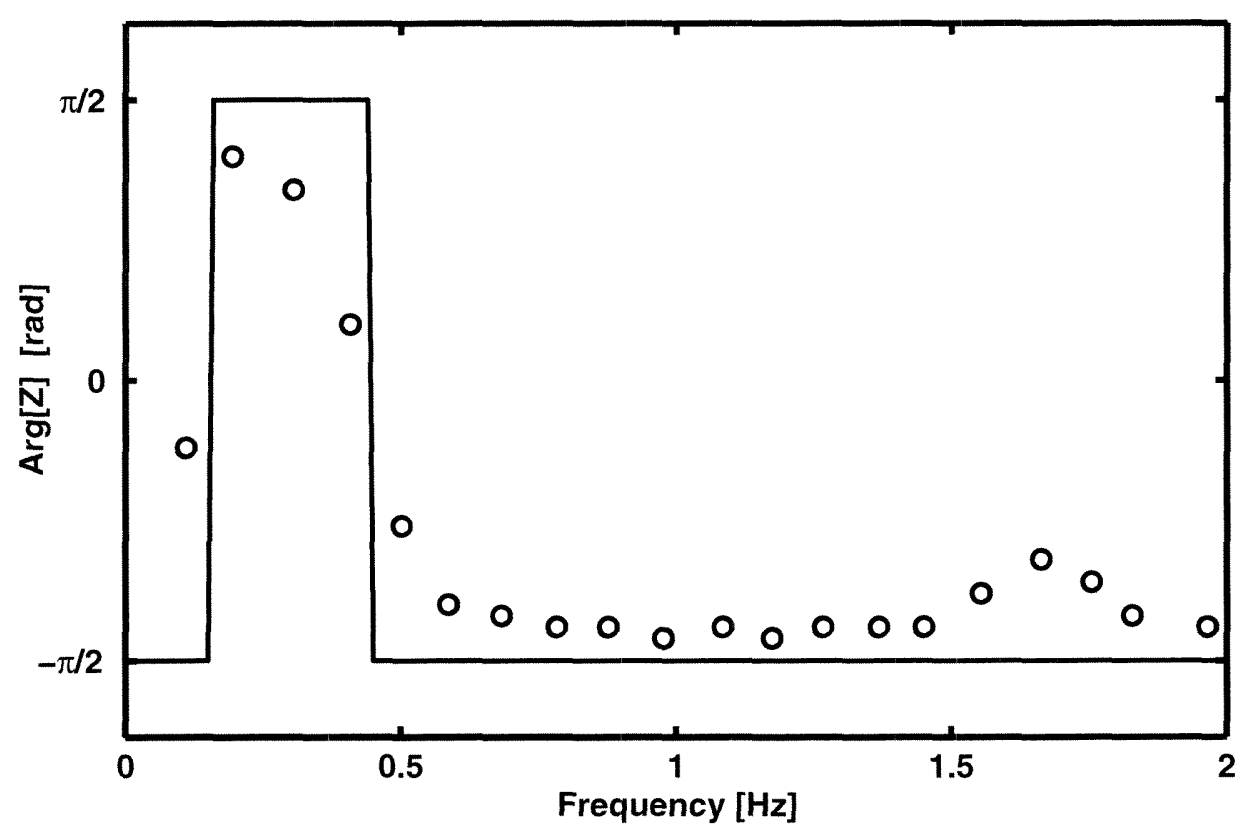

Figure 4.16: Phase of system impedance at low frequencies. The experimentally determined phase of the impedance, $\operatorname{Arg}[Z]$, is shown (O), and compared with the predictions of Equation 4.18 (-).

data at higher frequencies, phase angles could only be obtained reliably at low frequencies. The phase experiments were conducted with the oscillating piston setup located on the floor of the laboratory and therefore below the test section. The facility pressure needed to achieve the pressure balance across the piston was lower than for the forced oscillation experiments where the piston was located above the test section. The value for the overflow tank compliance used here in conjunction with Equation 4.18 has again been adjusted accordingly, slightly shifting the location of the zero near $f=0.2 \mathrm{~Hz}$.

With this adjustment made, the predictions of Equation 4.18 offer reasonable agreement with the experimentally determined phase. In particular, both exhibit phase transitions at the low frequency zero and again at resonance. Of note is that the experimental data also shows a slight increase and decrease in phase through the frequency range $f=1.5-1.8 \mathrm{~Hz}$. Whether this trend is simply a result of flaws in the experimental method or represents a zero and resonance not captured by the model remains unknown. 


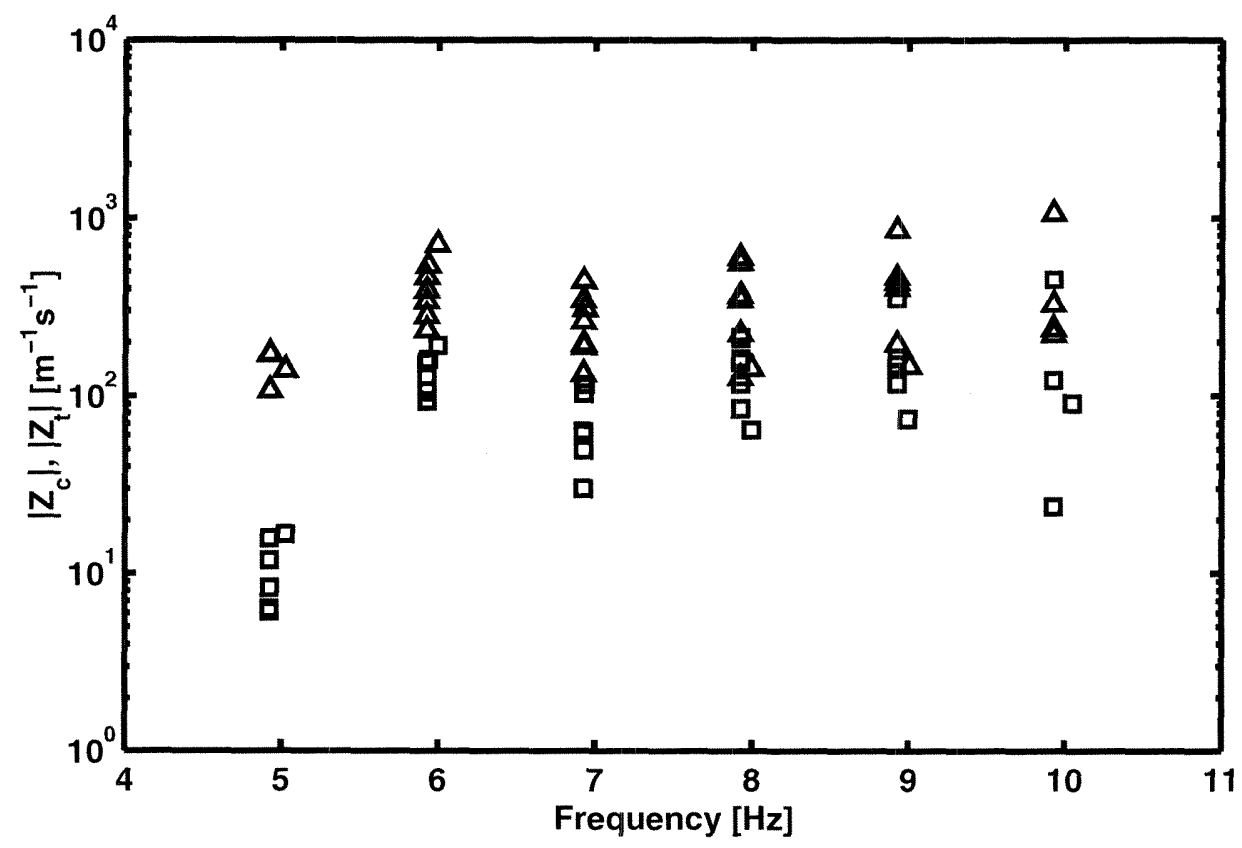

Figure 4.17: Magnitude of system impedance calculated from ceiling-mounted and test section centerline transducer measurements. $|Z|$, as determined from the ceiling-mounted $(\triangle)$ and test section centerline $(\square)$ transducer measurements.

\subsubsection{Test Section Inertance}

As indicated in Section 2.3, data was also obtained from a transducer mounted at the test section centerline. As exemplified by Figure 4.13, the data obtained from this transducer was generally very similar to the data obtained from the ceiling-mounted transducer. At higher frequencies, though, the amplitude of the fluctuating pressure at the test section centerline transducer was less than that at the ceiling-mounted transducer. Figure 4.17 shows a comparison of the ceiling-mounted and centerline transducer data for the higher frequencies. There is again a great deal of scatter in the data for the reasons discussed earlier, but it is clear that the values of the system impedance calculated based on the ceiling-mounted transducer measurements are higher than those based on the centerline transducer.

This can be attributed to the inertance and resistance of the test section length between the two transducers. The fact that, as shown in Figure 4.13, there is no substantial phase difference between the fluctuating pressures measured by the two transducers im- 
plies that this loss is a purely inertive one. In this case,

$$
\left|Z_{c}\right|-\left|Z_{t}\right|=\frac{\left|\tilde{p}_{c}\right|-\left|\tilde{p}_{t}\right|}{\left|\tilde{m}_{c t}\right|}=\omega L_{c t}
$$

where $Z_{c}$ is the system impedance based on the ceiling-mounted transducer measurements, $Z_{t}$ the system impedance based on the centerline transducer measurements, $L_{c t}$ the inertance of the length of test section between the two transducers, and $\tilde{m}_{c t}$ the fluctuating mass flow rate from $c$ to $t$.

Here, it is appropriate to make an adjustment to account for the fact that the mass flow rate passing locations $c$ and $t$ is only a fraction of the total mass flow rate entering the facility from the piston. A reasonable approximation of this fraction, especially at higher frequencies where the overflow tank receives little of the incoming excitation flow, is given by

$$
\tilde{m}_{c t}=\tilde{m}_{c}=\tilde{m}_{t}=\alpha \tilde{m}_{e},
$$

where $\alpha$ is determined by the relative impedance of the tunnel upstream and downstream to be

$$
\alpha=\frac{L_{t d}}{L_{t u}+L_{t d}} .
$$

With this adjustment, the average inertance based on the data of Figure 4.17 is $L_{c t}=$ $7 \mathrm{~m}^{-1}$, offering reasonable agreement with the value based on the geometry of the tunnel test section and transducers, $L_{c t}=5 \mathrm{~m}^{-1}$. The higher impedance observed experimentally can perhaps be attributed to the fact that the centerline transducer was not mounted flush with the interior test section walls but was instead located at the end of a short length of rigid tubing. The inertance of the fluid in this tubing would add to the test section inertance inferred from the experimental data. 


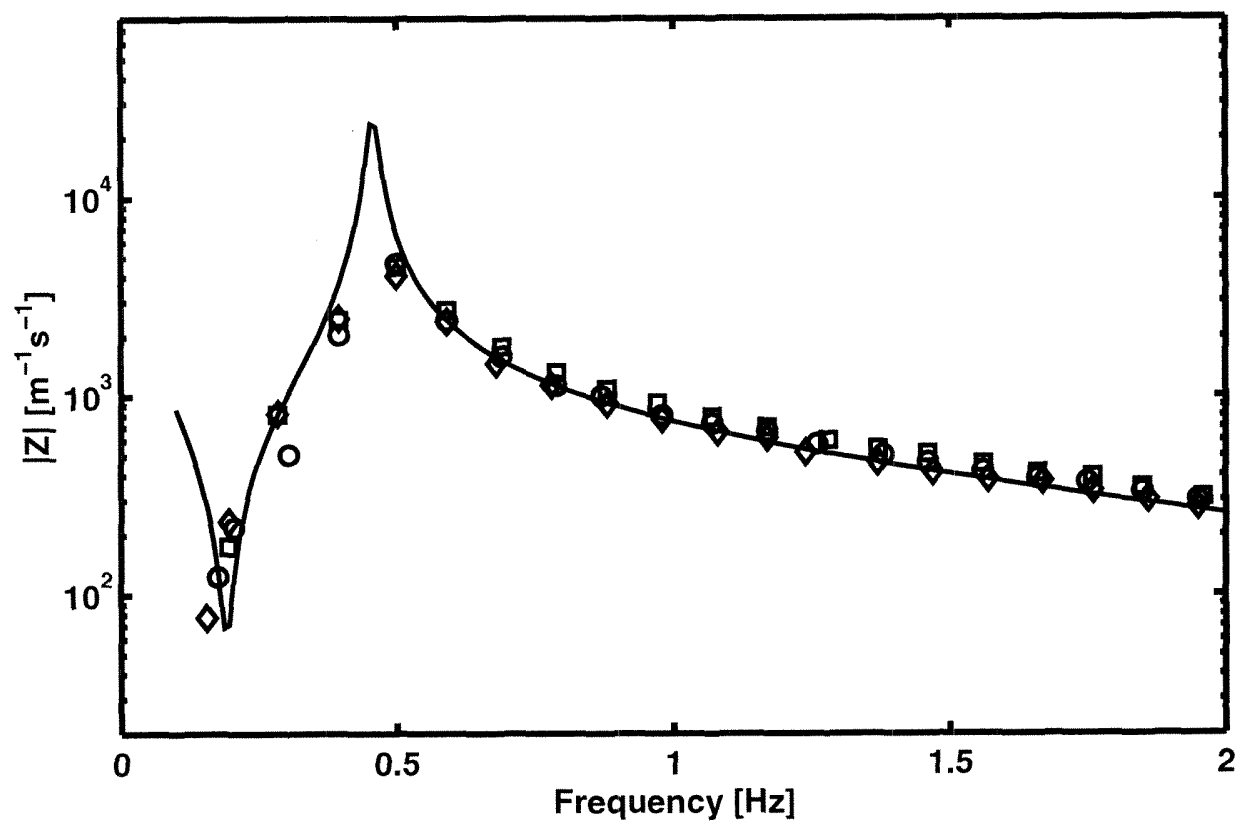

Figure 4.18: Effect of resistance on system impedance at low frequencies. The experimentally determined magnitude of the system impedance is repeated from Figure 4.14. Equation $4.18(-)$ is plotted for resistive values of $R_{c}=60$ and $R_{t}=20 \mathrm{~m}^{-1} \mathrm{~s}^{-1}$.

\subsubsection{Resistances}

In all of the preceding comparisons between experimental data and the predictions of Equation 4.18, the experimental data exhibits markedly less pronounced peaks in the magnitude of response near resonance, and less pronounced troughs near the two zeros. This is due to the fact that all resistances have been neglected in the preceding comparisons. Choosing values for the resistances to remedy this discrepancy and best fit the experimental data is difficult. Due to the discrete nature of the frequencies at which experiments were conducted, it is uncertain whether or not the precise resonance or zero may have been observed during the experiments. This difficulty is compounded by the scatter in the experimental data.

Nonetheless, Figures 4.18 and 4.19 show the effects of including reasonable estimates for the connecting pipe and tunnel resistances. Because the connecting pipe resistance principally affects the response at low frequencies, and the tunnel resistance affects the response at high frequencies, these two values could be chosen independently of one another to optimize the agreement at the zeros at $f=0.2$ and $4.0 \mathrm{~Hz}$, respectively. Even 


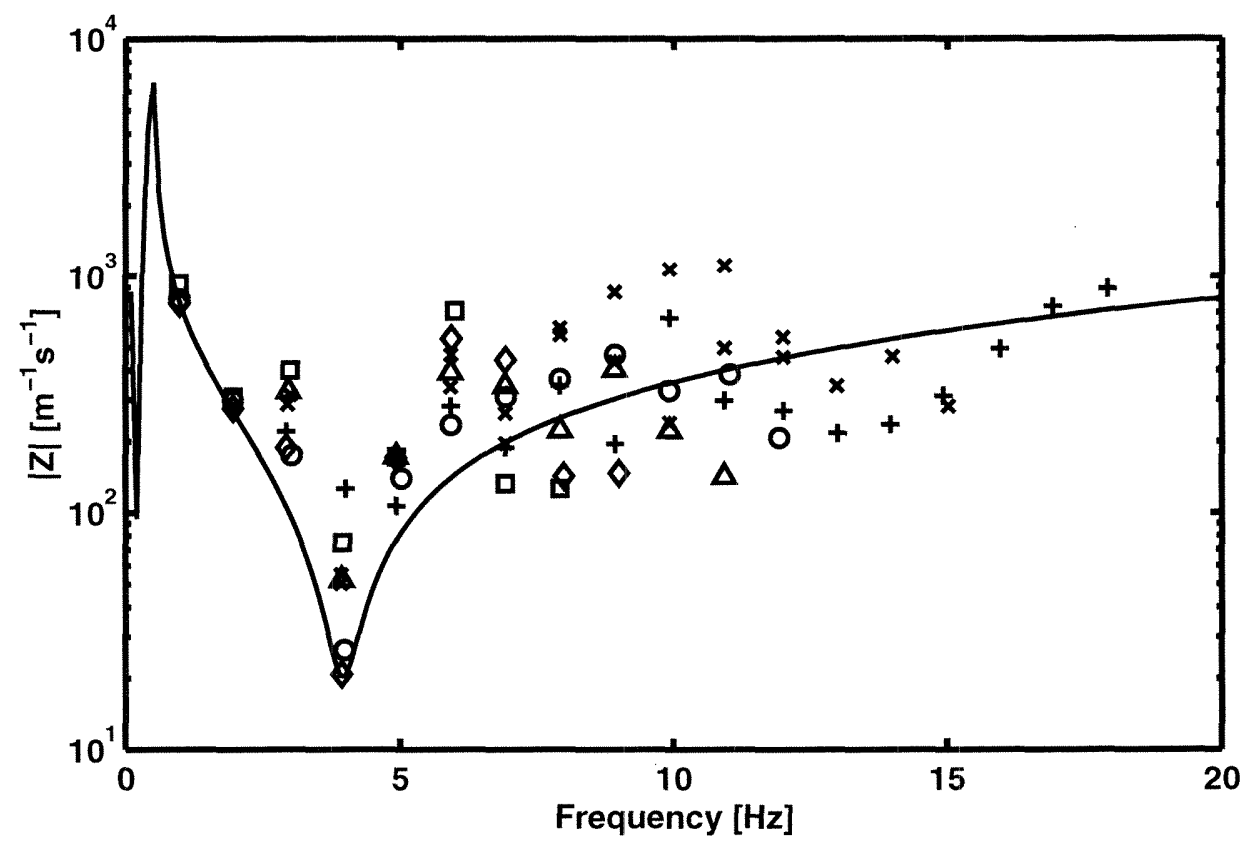

Figure 4.19: Effect of resistance on system impedance at high frequencies. The experimentally determined magnitude of the system impedance is repeated from Figure 4.15. Equation $4.18(-)$ is plotted for resistive values of $R_{c}=60$ and $R_{t}=20 \mathrm{~m}^{-1} \mathrm{~s}^{-1}$.

with these values of the resistances incorporated, there remains a substantial difference between the predicted and observed magnitude of response near resonance.

The values obtained from this fit to the experimental data are recorded in Table 4.3, and exceed the initial estimates in Table 4.2. As was the case with the tunnel inertance, determining resistances in this manner offers little insight regarding the precise distribution of these resistances within the facility. As discussed in Section 4.1.4, the resistances would seemingly be located in areas of smallest cross-section. It is surprising, then, that the comparison between the ceiling-mounted and test section centerline transducer data suggested that the test section resistance is very small.

\subsubsection{Effect of Mean Flow}

The forced response experiments described above were also repeated in the presence of a mean flow, and the behavior of the system response remained largely unchanged. However, the decrease in the magnitude of response seen at the zero near $f=4.0 \mathrm{~Hz}$ in Figure 4.15 became somewhat less pronounced. This indicates that the tunnel resistance may in fact 


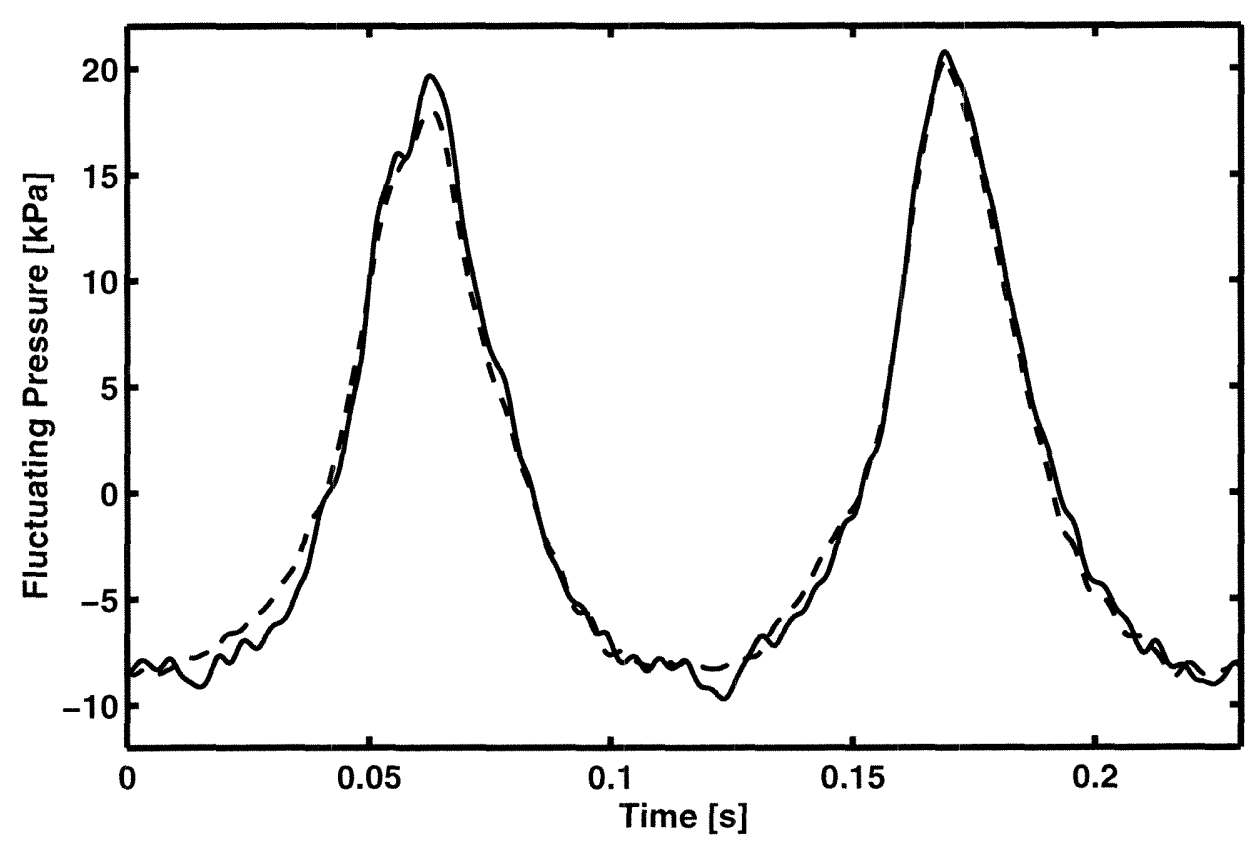

Figure 4.20: Floor- and ceiling-mounted transducer measurements. The solid line ( - ) and dashed line (--) show simultaneous pressure measurements obtained from the floorand ceiling-mounted pressures transducers, respectively. Both signals have been low-pass filtered at $200 \mathrm{~Hz}$.

be higher still in the presence of a mean flow, as suggested in Section 4.1.4.

\subsection{Far Field Pressure Attenuation}

The validity of the proposed one-dimensional model for the facility dynamics can be further assessed by considering the pressure measurements obtained from the floor- and ceiling-mounted pressure transducers during the instability cycle. Figure 4.20 shows a typical set of simultaneous pressure measurements obtained from the two transducers. A further failing of the three-dimensional model given in Section 3.3.8 is immediately apparent in that the two signals have comparable amplitudes. This is inconsistent with the nearly threefold attenuation predicted by Equation 3.9 and the transducer distances of $\mathcal{R}_{f}=0.15 \mathrm{~m}$ and $\mathcal{R}_{c}=0.43 \mathrm{~m}$.

The model for the facility dynamics predicts a pressure difference associated with the inertive losses between the two transducers. This difference in the transducer measure- 
ments $\tilde{p}_{f}$ and $\tilde{p}_{c}$ is given by

$$
\left|\tilde{p}_{c}\right|=\left|\tilde{p}_{f}\right|-\omega L_{f c}\left|\tilde{m}_{f c}\right|
$$

where $\left|\tilde{m}_{f c}\right|$ is the amplitude of the fluctuating mass flow rate between the transducers and $L_{f c}$ is the inertance of the test section between the two transducers. Specifically,

$$
L_{f c}=\frac{l_{f c}}{A_{t}},
$$

where $l_{f c}$ is the distance between the transducers and $A_{t}$ the cross-sectional area of the test section.

As implied by Equation 4.1, the mass flow rate $\tilde{m}_{f c}$ is related to the fluctuating cavity volume on the propeller, such that

$$
\left|\tilde{p}_{f}\right|-\left|\tilde{p}_{c}\right|=\alpha \rho \omega^{2} L_{f c}\left|\tilde{V}_{c a v}\right|
$$

Here, the factor $\alpha$ developed in Section 4.3.4 has been included to reflect the fact that not all of the mass flow rate generated by the fluctuating cavity volume flows upstream towards the ceiling-mounted transducer. The amplitude of the pressure difference predicted by the model is then $\left|\tilde{p}_{f}\right|-\left|\tilde{p}_{c}\right|=519 \mathrm{~Pa}$ at a typical instability frequency of $f=10 \mathrm{~Hz}$.

This pressure difference compares favorably with that shown in Figure 4.20. In fact, averaging over eight transducer measurements of approximately forty instability cycles each, the experimentally observed pressure difference is found to be $\left|\tilde{p}_{f}\right|-\left|\tilde{p}_{c}\right|=487 \mathrm{~Pa}$. Also encouraging is the attenuation of the high frequency content between the floor- and ceiling-mounted transducer signals of Figure 4.20. This is consistent with the scaling of the pressure difference with frequency given in Equation 4.33 . 


\section{Chapter 5}

\section{Cavitation Dynamics}

The analysis and experiments of Chapter 4 demonstrate that the conditions within the facility, in particular those in the test section, will respond to the volumetric excitations imposed by the fluctuating cavity volume of the instability. Yet the results presented in Chapter 3 indicate that the cavity volume itself responds to changes in these very same test section conditions. Clearly, then, the facility dynamics and the cavitation dynamics must be considered as part of a coupled dynamic system. Essential to understanding these coupled dynamics, and not yet properly quantified, is how the cavity volume on the propeller blades responds to these variations in test section conditions.

\subsection{Development of Cavitation Dynamic Parameters}

As mentioned in Section 3.3.6, it was suggested by Tulin (1953) following studies of two-dimensional hydrofoils that the non-dimensional cavity length, $\frac{l_{t}}{c}$, is approximately a function of the ratio of the effective angle of attack of the propeller blade to the cavitation number, $\frac{\alpha}{\sigma}$. Recalling also the observation of Blake (1986) that the cavity volume on a propeller blade is proportional to the propeller radius and the square of the cavity length, it is clear that the total cavity volume is also approximately a function of the ratio $\frac{\alpha}{\sigma}$.

The discussion of Section 3.1 demonstrates that at a given propeller rotation speed, the effective angle of attack $\alpha$ is determined entirely by the mass flow rate in the tunnel test section, $m_{t s}$, while the cavitation number is determined entirely by the test section pressure $p_{t}$. It is therefore appropriate (Brennen 1994) to express the quasistatic variation 
in cavity volume with $\alpha$ and $\sigma$ in terms of a cavitation compliance, $K^{*}$, and a mass flow gain factor, $M^{*}$, defined as

$$
\begin{aligned}
K^{*} & =-\rho\left(\frac{d V_{c a v}}{d p_{t}}\right)_{m_{t s}} \\
M^{*} & =-\rho\left(\frac{d V_{c a v}}{d m_{t s}}\right)_{p_{t}} .
\end{aligned}
$$

It is convenient to recast these expressions in terms of the cavitation number and advance ratio. From Equations 3.1 and 3.4, it can be shown that

$$
\begin{aligned}
K^{*} & =-\frac{2}{\Omega^{2} R^{2}}\left(\frac{d V_{c a v}}{d \sigma}\right)_{J} \\
M^{*} & =-\frac{\pi}{A_{t s} \Omega R}\left(\frac{d V_{c a v}}{d J}\right)_{\sigma},
\end{aligned}
$$

where $\Omega$ is the angular frequency of propeller rotation, $R$ the radius of the propeller, and $A_{t s}$ the cross-sectional area of the test section. The parameters $K^{*}$ and $M^{*}$ thus summarize the response of the cavity volume to variations in upstream pressure and mass flow rate, respectively. The cavitation compliance is a measure of the compressibility of the cavitating flow, and the mass flow gain factor reflects the extent to which variations in mass flow rate are either consumed or augmented by the changing cavity volume.

\subsection{Estimation of Cavitation Dynamic Parameters}

Equations 5.3 and 5.4 indicate that it is possible to obtain values for $K^{*}$ and $M^{*}$ from experiments determining the cavity volume at various advance ratios and cavitation numbers. Such an estimate of cavity volumes at various test section conditions can be obtained by again recalling the suggestion of Blake (1986) for evaluating cavity volume and applying this method to the cavitation extent measurements presented in Section 3.2.1. Figure 5.1 shows the results of this calculation for the cavitation extent measurements obtained at moderate propeller rotation speed.

Also shown in Figure 5.1 are several lines corresponding to constant values of the

parameter $\xi=\frac{J_{o}-J}{\sigma}$ developed in Section 3.3.6. Not surprisingly, lines of constant $\xi$ 


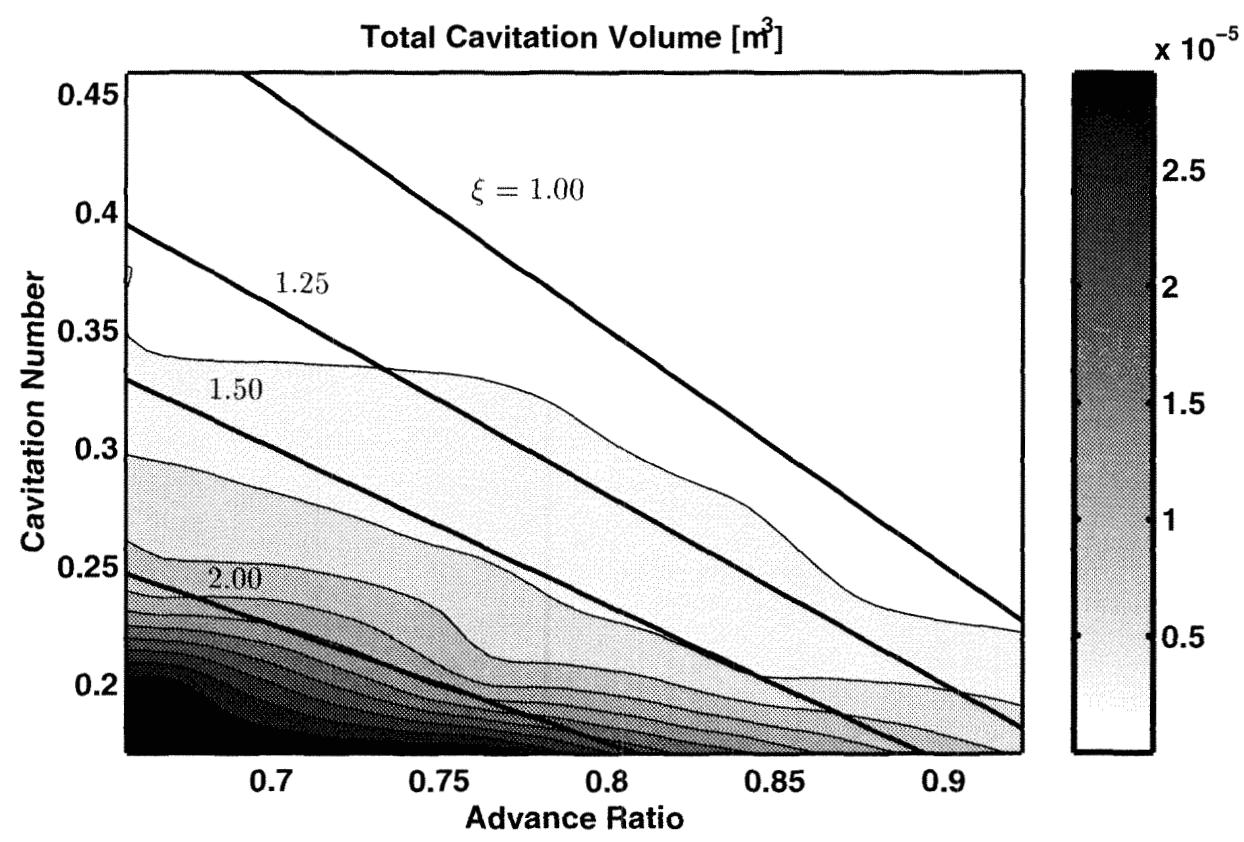

Figure 5.1: Cavity volume on propeller blades at moderate rotation speed. $V_{c a v}$ as computed from cavity length measurements using the method of Blake (1986). The propeller rotation speed was $n=28.3 \mathrm{~Hz}$.

correspond quite closely to contours of constant cavity volume. It is convenient to fit the cavity volume to a function of the form $V_{c a v}=h(\xi)$, with a simple second order polynomial $h(\xi)=a \xi^{2}+b \xi+c$ providing acceptable results when $a=0.86 \times 10^{-5} \mathrm{~m}^{3}$ and $b=-1.2 \times 10^{-5} \mathrm{~m}^{3}$.

Combining the results of this polynomial fit with Equations 5.3 and 5.4 yields expressions for the cavitation compliance and mass flow gain factor,

$$
\begin{aligned}
K^{*} & =\frac{2 \xi}{\Omega^{2} R^{2} \sigma} \frac{d h}{d \xi} \\
M^{*} & =\frac{\pi}{A_{t s} \Omega R \sigma} \frac{d h}{d \xi} .
\end{aligned}
$$

Figure 5.2 shows the results of this evaluation for moderate propeller rotation speeds. Shown in Figure 5.3 are the results of this process conducted with the data taken at a higher rotation speed. In general, the cavitation compliance and mass flow gain factor increase steadily with decreasing advance ratio and cavitation number. The behavior of the mass flow gain factor at higher advance ratios in Figure 5.3 is very irregular, but the 

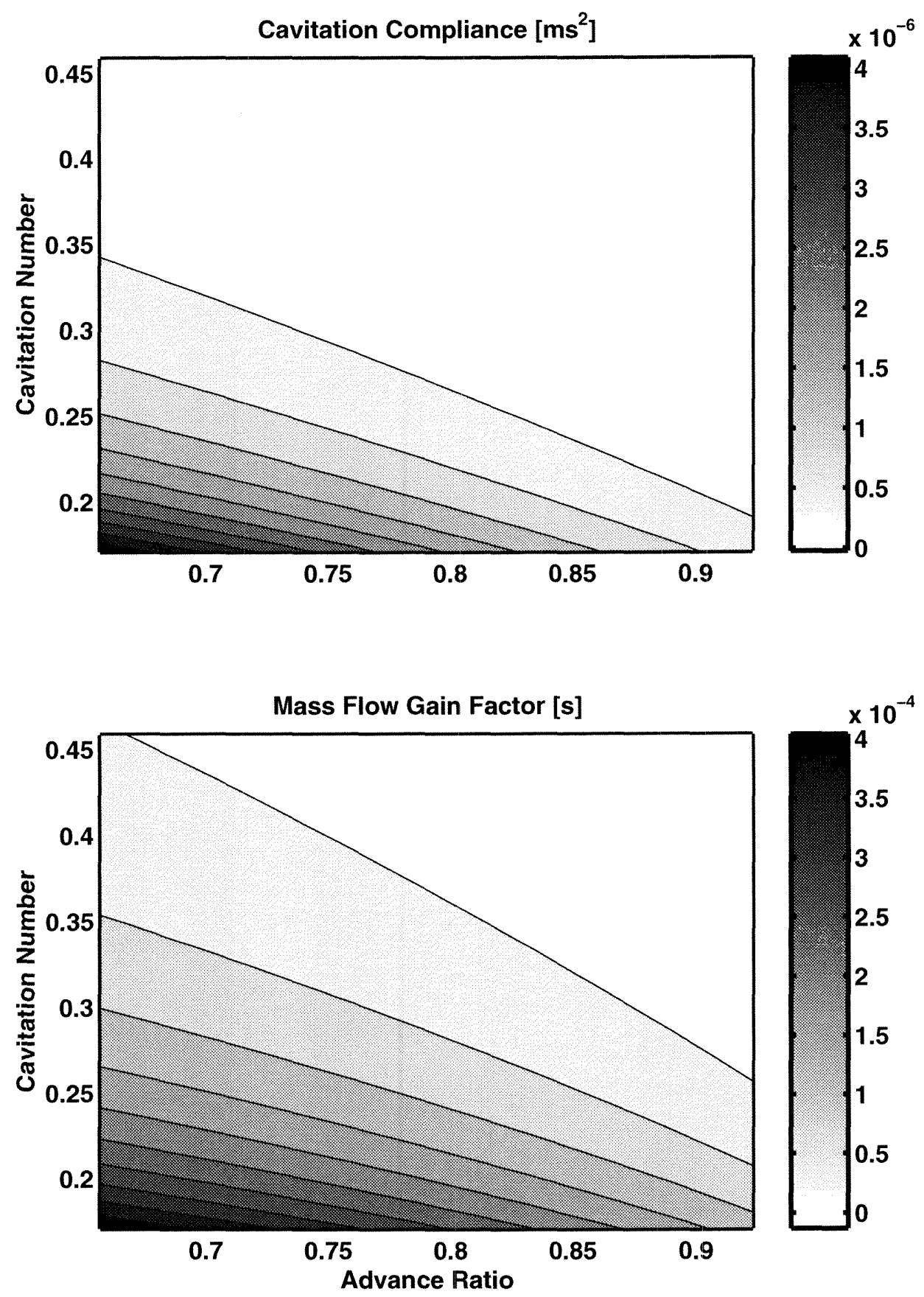

Figure 5.2: Cavitation compliance and mass flow gain factor estimates at moderate propeller rotation speed. $K^{*}$ and $M^{*}$ computed using the method summarized in Equations 5.5 and 5.6. The propeller rotation speed was $n=28.3 \mathrm{~Hz}$. 

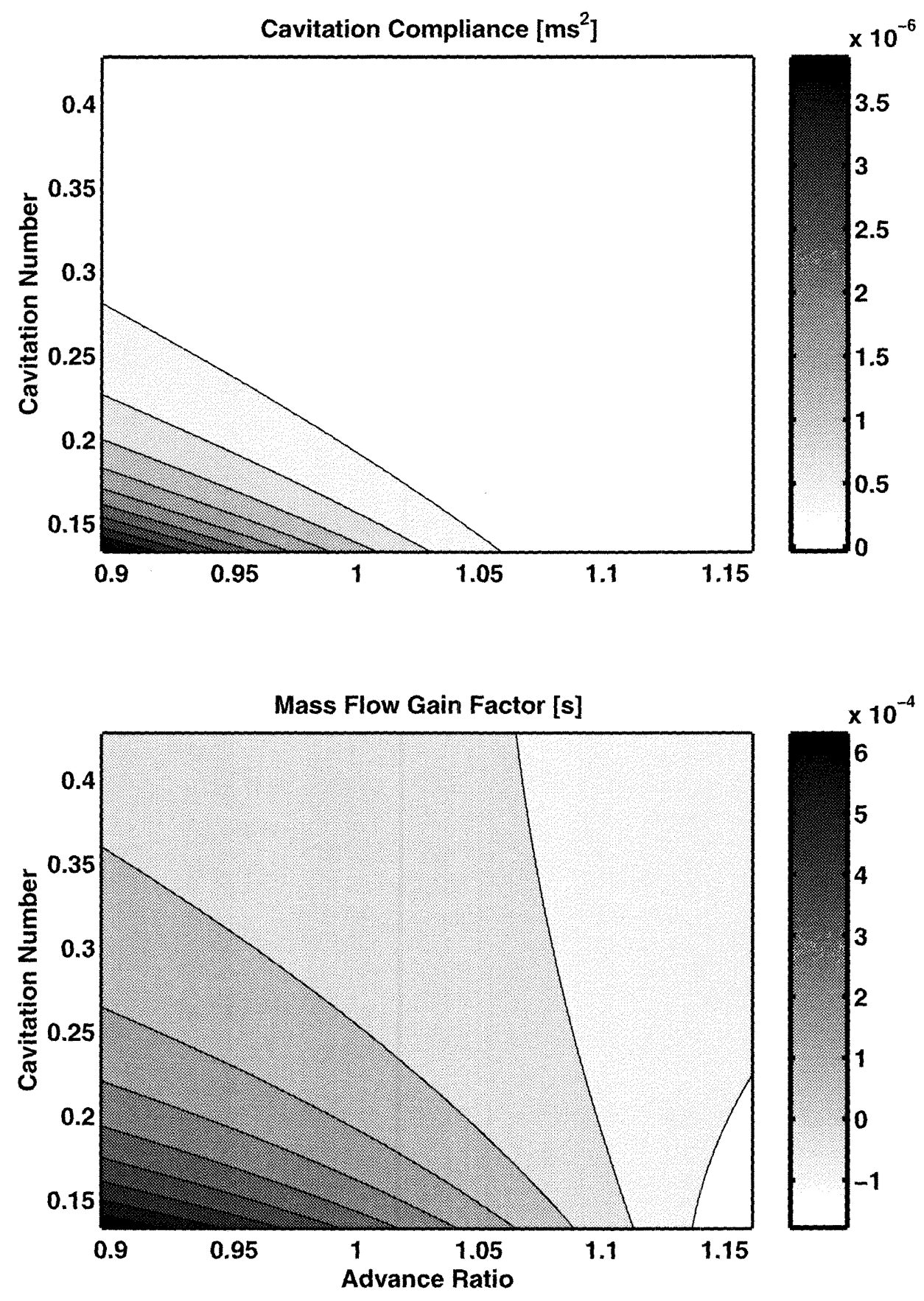

Figure 5.3: Cavitation compliance and mass flow gain factor estimates at high propeller rotation speed. $K^{*}$ and $M^{*}$ computed using the method summarized in Equations 5.5 and 5.6. The propeller rotation speed was $n=31.7 \mathrm{~Hz}$. 
accuracy of the calculation in this region is poor due to the behavior of the parameter $\xi$ near the design advance ratio of $J_{o}=1.15$.

It is worth repeating that the estimates of the cavitation dynamic parameters are quasistatic estimates. However, the reduced frequency of $k=0.07$ computed in Section 3.3.4 indicates that the variations in cavitation during the instability cycle occur over a time scale that is longer than the time required for disturbances to propagate across the propeller blade. Furthermore, the experiments of Section 3.2.2 investigating the behavior of the propeller at yaw revealed that the dynamics of cavity growth and recession are considerably faster than the rotation frequency of the propeller. Since the instability frequency is lower still, the quasistatic estimates seem appropriate for modeling the dynamic behavior of the cavitation during the instability cycle.

\subsection{Comparison of Cavitation Dynamic Parameters}

To facilitate comparison with results obtained by previous investigators exploring the unstable behavior of cavitating pumps, the cavitation dynamic parameters can be nondimensionalized as

$$
\begin{aligned}
& K=\frac{\Omega^{2} K^{*}}{R} \\
& M=\Omega M^{*} .
\end{aligned}
$$

Also, it is necessary to account for the fact that in the case of a cavitating pump, the mass flow gain factor is based on the mass flow rate entering the cross-sectional area of the pump. In contrast, the definition of Equation 5.4 is based on the mass flowing through the cross-sectional area of the entire test section. An adjustment is therefore necessary,

increasing the mass flow gain factor computed for the propeller by a factor of $\eta=\frac{A_{t s}}{A_{x}}$, the ratio of the test section area to the area of the propeller disc.

After this adjustment, the ranges of the dimensionless cavitation compliance and dimensionless mass flow gain factor are determined to be $K=0.0-1.4$ and $M=0.05-0.20$ for the advance ratios and cavitation numbers investigated at $n=28.3 \mathrm{~Hz}$. At $n=$ $31.7 \mathrm{~Hz}$, these ranges increase slightly to $K=0.0-1.6$ and $M=0.0-0.4$. For com- 
parison, in the first theoretical calculation of these dynamic parameters for a cavitating inducer, Brennen and Acosta (1976) obtained values in the ranges of $K=0.05-0.2$ and $M=0.6-0.8$ for cavitation numbers above $\sigma=0.02$. Experimental measurements by Brennen, Meissner, Lo, and Hoffman (1982) for cavitating inducers at $\sigma=0.2$ yielded a larger typical value of $K=0.25$, but similar values of $M=0.6$. More recently, theoretical studies by Otsuka, Tsujimoto, Kamijo, and Furuya (1996) examined the potential frequency dependence of $K$ and $M$. At low frequencies and $\sigma=0.17$, they obtained values of the order of $K=0.06$ and $M=0.6$. These values offer reasonable agreement with those determined in the current work, since some differences would be expected given the geometrical differences between propellers and pumps.

Finally, it should be noted that the definitions of the dimensional parameters given in Equations 5.3 and 5.4, and therefore the dimensionless parameters of Equations 5.7 and 5.8, are based on the test section static pressure. In contrast, the values encountered in the cavitating pump literature are more commonly based on the total pressure upstream of the pump. However, reviewing the definitions of the cavitation compliance, mass flow gain factor, and total pressure indicates that

$$
\begin{aligned}
& K^{T}=K^{S} \\
& M^{T}=M^{S}-\lambda K^{S},
\end{aligned}
$$

where the superscripts $T$ and $S$ denote the values based on total pressure and static pressure, respectively. Here, the factor $\lambda$ is given by

$$
\lambda=\frac{\bar{u}_{t s} R}{A_{t s} \Omega} .
$$

Choosing a typical value for the mean test section flow velocity of $\bar{u}_{t s}=3.75 \mathrm{~ms}^{-1}$ yields a value for this parameter of $\lambda=0.02$. This value demonstrates that, for the cavitating propeller, the cavitation dynamic parameters based on total and static pressure may be used interchangeably. 


\subsection{Effect of Tip Vortex Participation}

As noted in the description of the propeller instability given in Chapter 3, the fluctuation in cavitation extent is not confined to the cavitation on the propeller blades, but also includes the tip vortices downstream of the propeller. The definition of the cavitation compliance given in Equation 5.3 indicates that the compliance scales linearly with the total cavity volume. Thus, the fluctuations in tip vortex volume suggest a higher value of the cavitation compliance than the calculations in Section 5.2, which are based only on the cavity volume on the propeller blades. Whether or not tip vortex participation affects the mass flow gain factor is less certain. The mass flow rate affects cavity volume only through changing the effective angle of attack of the propeller blades. Exactly how the tip vortex volume varies with changing effective angle of attack is not known, but it is likely that the mass flow gain factor is also somewhat higher than estimated in Section 5.2.

Quantifying the effect of the tip vortices on the cavitation compliance is difficult, as measuring the variation in tip vortex volume during the instability cycle is quite challenging. Furthermore, the fact that the vortices can persist in time through several instability cycles complicates efforts to determine the total vortex length participating in the volumetric fluctuations. This situation is simplified by noting from high speed movie footage that a substantial fluctuation of vortex diameter occurs over a distance $l_{d}=0.50 \mathrm{~m}$ downstream from the propeller. Further downstream, the vortices are more poorly defined and the magnitude of fluctuation declines rapidly. Considering the helical geometry of the vortices, the participating vortex length is given by

$$
l_{v}=N l_{d}\left[1+\left(\frac{2 \pi n R}{U_{w}}\right)^{2}\right]^{\frac{1}{2}}
$$

where $U_{w}$ is the velocity of the propeller wash, a quantity greater than the upstream tunnel velocity, $U_{t}$. Determining a value of $U_{w}=5.5 \mathrm{~ms}^{-1}$ from high speed movie footage and using a typical rotation speed of $n=30.8 \mathrm{~Hz}$ yields a participating vortex length of $l_{v}=10.5 \mathrm{~m}$. Also obtained from the movie footage is the variation in vortex cavity diameter during the instability cycle, approximately $D_{v}=2.0-7.5 \mathrm{~mm}$. These minimum and maximum diameters and the participating vortex length imply a vortex cavity volume 
fluctuation of $\left|\tilde{V}_{v}\right|=2.1 \times 10^{-4} \mathrm{~m}^{3}$. This is much larger than the previously calculated volumetric fluctuation on the propeller blades, $\left|\tilde{V}_{\text {cav }}\right|=4.2 \times 10^{-5} \mathrm{~m}^{3}$, potentially increasing the cavitation compliance by as much as a factor of six. Implicit in this calculation is that the vortex volume responds to fluctuating pressures in the same manner as the cavitation on the propeller blades. This is probably not accurate. Nevertheless, the approximation does demonstrate that the vortex volume fluctuations are potentially very significant.

It should be noted that including the tip vortex contributions would alter the calculations made in previous chapters that were based on the fluctuating volume of $\left|\tilde{V}_{c a v}\right|=$ $4.2 \times 10^{-5} \mathrm{~m}^{3}$. Most notably, the calculation in Section 4.3 .4 of the test section inertance between the floor- and ceiling-mounted transducers, and the analysis in Section 4.4 of the pressure attenuation between these two transducers are changed substantially. In both cases, the agreement between the experimentally and analytically determined quantities is diminished considerably. However, as the exact extent of tip vortex participation in the volumetric fluctuation remains uncertain, these previous calculations remain based on the more precisely determined fluctuating volume on the propeller blades. 


\section{Chapter 6}

\section{Combined Facility and Cavitation Dynamics}

Chapters 4 and 5 explored separately the dynamics of the facility and the dynamics of the propeller cavitation. Considered independently, neither the facility nor the cavitation dynamics offer any insight into the origins of the observed instability. In this chapter, the two dynamic behaviors are considered together as part of a more complete model, and the origins of the instability are explored.

\subsection{Passive and Active Dynamics}

\subsubsection{System Impedance}

The facility dynamics developed in Chapter 4 are characterized by resistive, inertive, and compliant behaviors. Systems of this nature are termed passive systems, in that any fluctuations within the system will be dissipated in time without an external input of fluctuation energy. This behavior can be characterized mathematically based on the system impedance, $Z$. For all passive systems, $\operatorname{Re}[Z] \geq 0$, with equality achieved only in the case of a system with no resistance.

The inclusion of the cavitation dynamics significantly alters the character of the overall system dynamics. The nature of this change is most clearly illustrated by considering the simplified system shown in Figure 6.1, consisting only of a source of fluctuating mass flow rate and the cavitation dynamics discussed in Chapter 5 . Recalling the definitions of the 


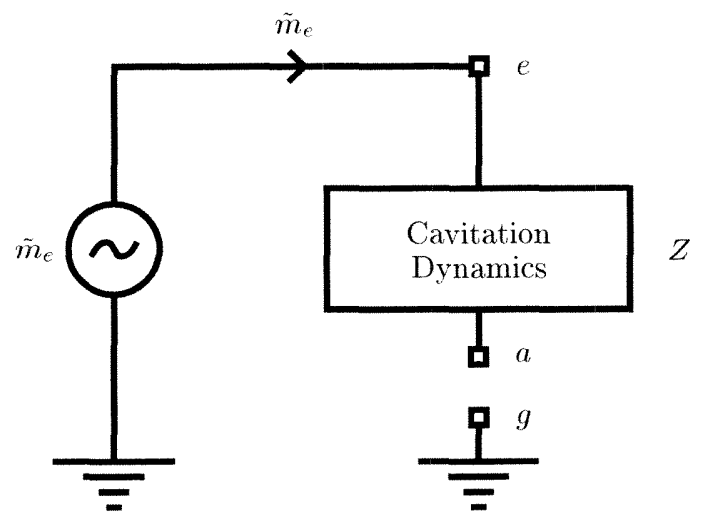

Figure 6.1: Schematic of system including only cavitation dynamics.

cavitation dynamic parameters, a control volume analysis yields

$$
\begin{gathered}
\tilde{p}_{a}^{T}=\tilde{p}_{e}^{T} \\
\tilde{m}_{a}=\tilde{m}_{e}-j \omega K^{*} \tilde{p}_{e}^{T}-j \omega M^{*} \tilde{m}_{e} .
\end{gathered}
$$

Because the fluctuating mass flow rate at station $a$ is zero, the system impedance can be evaluated as

$$
Z=\frac{1-j \omega M^{*}}{j \omega K^{*}}
$$

More importantly,

$$
\operatorname{Re}[Z]=-\frac{M^{*}}{K^{*}}
$$

Recalling from Section 5.2 that the cavitation compliance and mass flow gain factor of the propeller cavitation are both positive, it is clear that this simplified system is characterized by a negative value of the real part of the system impedance. The simplified system is thus an active system in that it is capable of sustaining fluctuations without an external input of fluctuation energy, instead extracting the fluctuation energy from the steady flow. This is in accord with the view of other investigators such as Brennen (1978) and Tsujimoto, Kamijo, and Yoshida (1993) who characterize cavitating pump instabilities as essentially 
arising from a positive mass flow gain factor.

\subsubsection{Fluctuation Energy Flux}

The active dynamics concept can also be cast in terms of the flux of fluctuation energy through a location within the facility. As noted by Brennen (1994), the total energy flux

entering a location is given by $E^{T}=\frac{m p^{T}}{\rho}$. The portion of this energy flux associated with the fluctuating flow quantities, the fluctuation energy flux, is given by

$$
E=\frac{1}{4 \rho}\left(\tilde{m} \tilde{\tilde{p}}^{T}+\tilde{\tilde{m}} \tilde{p}^{T}\right)
$$

In the case of location $e$ in the systems shown in Figures 4.1 and 6.1 , this quantity represents the net fluctuation energy provided to the system. If this quantity is negative, the system is an active system and a source of fluctuation energy.

Not surprisingly, then, if the definition of the system impedance given in Equation 4.4 is substituted into Equation 6.5, it is apparent that

$$
E=\frac{\tilde{m} \tilde{\bar{m}}}{4 \rho}(\bar{Z}+Z)
$$

and therefore

$$
E \sim \operatorname{Re}[Z]
$$

Thus, a system classified as active due to a negative real part of the system impedance is also active by virtue of being a source of fluctuation energy.

\subsection{Local Impedance}

Before the combined cavitation and facility dynamics can be analyzed, it is necessary to address the possible effects of the local inertance associated with the expansion and contraction of the cavity volume on the propeller blades. The nature of this inertance can be understood by considering a simple spherical cavity as shown in Figure 6.2. As the cavity of radius $R$ expands and contracts, it creates a fluctuating mass flow rate, 


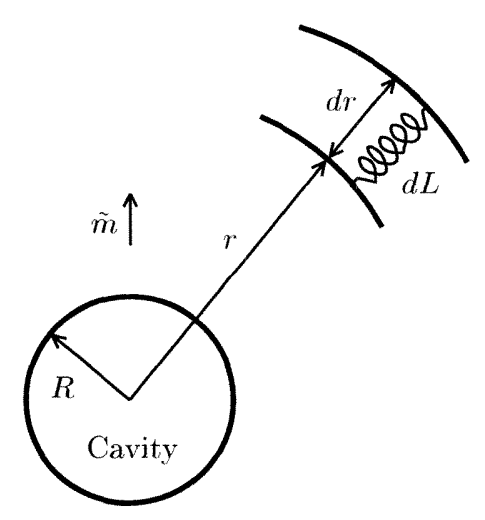

Figure 6.2: Schematic of local impedance.

$\tilde{m}$, traveling radially outward from the cavity. The associated pressures in the flow are attenuated with increasing radius, due to the inertive nature of the fluid mass beyond the cavity surface. By applying a control volume analysis to the spherical shell geometry of Figure 6.2 , it can be seen that

$$
d \tilde{p}=j \omega \tilde{m} \frac{d r}{4 \pi r^{2}}
$$

Integrating this differential pressure drop from the cavity surface to the far field yields an expression for the pressure attenuation of

$$
\tilde{p}_{R}-\tilde{p}_{\infty}=j \omega L_{l} \tilde{m}
$$

where $\tilde{p}_{R}$ and $\tilde{p}_{\infty}$ are the fluctuating pressures at the cavity surface and in the far field, respectively. In this expression, the local inertance, $L_{l}$, is given by

$$
L_{l}=\frac{1}{4 \pi R}
$$

Equations 6.9 and 6.10 are together equivalent to Equation 3.9, the expression for the far field pressure predicted by the three-dimensional model. The local inertance thus incorporates the locally three-dimensional effects into the one-dimensional model of the facility dynamics. The result is the combined one- and three-dimensional approach described in 
Section 3.3.8.

Choosing an appropriate cavity radius for the propeller cavitation is difficult, as the cavity shape on the propeller blades is far from the spherical configuration assumed in Figure 6.2. Furthermore, any such cavity radius changes dramatically throughout the instability cycle. The actual local inertance is therefore time variant, with any value obtained from Equation 6.10 representing a mean value. Nonetheless, an estimate of the local inertance can be obtained by assuming that the total cavity volume behaves as a spherical source when viewed from a sufficient distance in the far field. Based on the cavity volume estimates of Section 3.3.8, a mean cavity volume of $\bar{V}_{c a v}=5.0 \times 10^{-5} \mathrm{~m}^{3}$ is obtained, implying a mean cavity radius of $\bar{R}_{c a v}=23 \mathrm{~mm}$ and a local inertance of $L_{l}=3.5 \mathrm{~m}^{-1}$.

\subsection{Modeling of the System}

\subsubsection{Full Model}

Figure 6.3 shows a schematic representation of the combined facility and cavitation dynamics. Here, point $e$ is taken to be a location within the test section beyond the effects of the local inertance but near enough to the propeller that the majority of the tunnel inertance lies exterior to the portion of the test section between the propeller and point $e$. Because the cavitation dynamic parameters of Chapter 5 were determined with pressure measurements from manometer taps at such a location, it is possible to write a set of equations governing the behavior of the flow at point $e$ without reference to the local inertance. Specifically,

$$
\begin{gathered}
\tilde{p}_{e}^{T}=\tilde{p}_{u}^{T} \\
\tilde{m}_{e}=\tilde{m}_{u}-j \omega K^{*} \tilde{p}_{u}^{T}-j \omega M^{*} \tilde{m}_{u},
\end{gathered}
$$

where $\tilde{p}_{u}^{T}, \tilde{p}_{e}^{T}, \tilde{m}_{u}$, and $\tilde{m}_{e}$ are the fluctuating total pressures and mass flow rates at points $u$ and $e$, respectively. In this sense, the cavitation dynamic parameters developed in Chapter 5 already incorporate the effects of the local inertance, and the difficulties of 


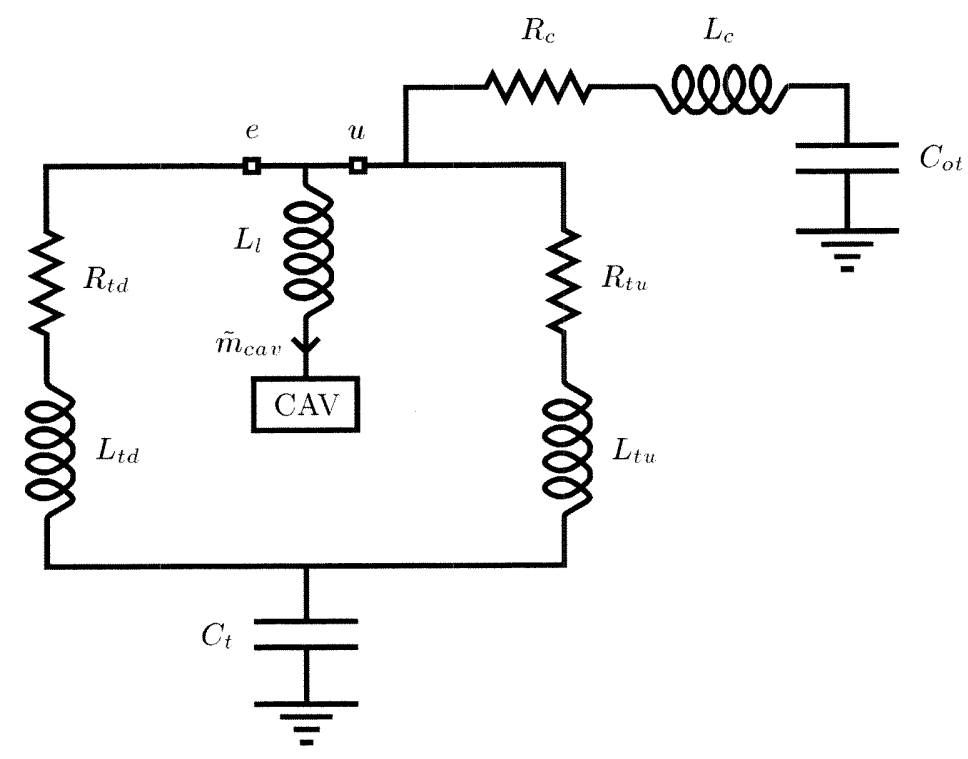

Figure 6.3: Schematic of facility and cavitation dynamics.

Section 6.2 in estimating this parameter are immaterial.

Employing Equations 6.11 and 6.12 in conjunction with the standard rules for simplifying resistive, inductive, and capacitive networks allows for the solution of the system impedance at point $e$. The impedance is given by

$$
Z=\frac{\left(L_{t} C_{t} \omega^{2}-1\right) Z_{c}}{j L_{t} C_{t}\left[Z_{c} K^{*}-M^{*}\right] \omega^{3}-L_{t} C_{t}\left[\frac{Z_{c} M^{*}}{L_{t u}}-1\right] \omega^{2}+j\left[M^{*}-Z_{c}\left(C_{t}+K^{*}\right)\right] \omega-1}
$$

where $L_{t}^{-1}=L_{t u}{ }^{-1}+L_{t d}^{-1}$ and $Z_{c}$ is the impedance of the connecting pipe and overflow tank,

$$
Z_{c}=j \omega L_{c}+R_{c}+\frac{1}{j \omega C_{o t}}
$$

Figure 6.4 shows the real part of this system impedance at various frequencies. In generating this plot, values for $K^{*}$ and $M^{*}$ were obtained from the calculations presented in Figures 5.2 and 5.3 at the typical instability conditions of $n=30.8 \mathrm{~Hz}, J=0.64$ 


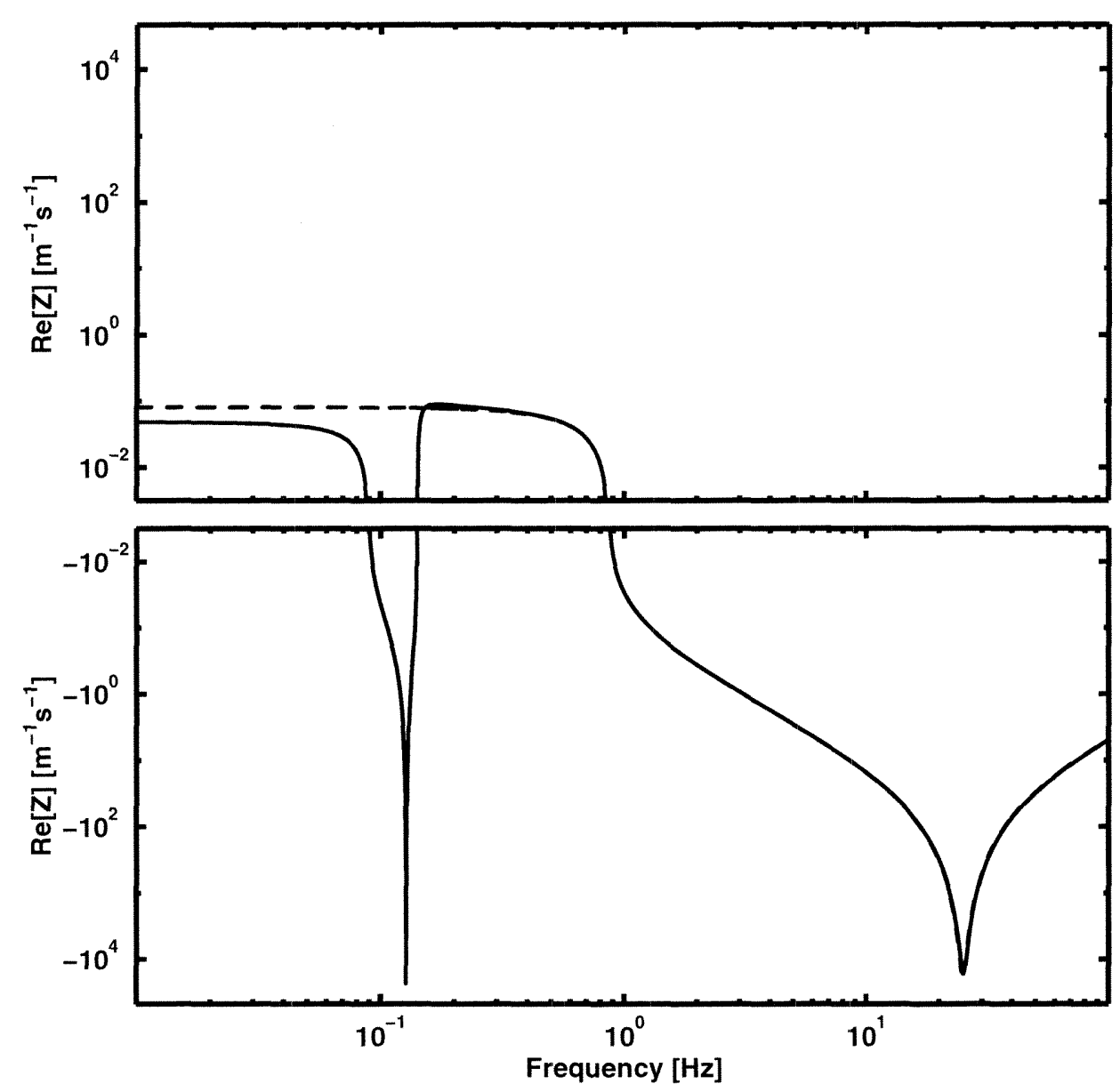

Figure 6.4: Real part of system impedance for combined dynamics model. The results of the full model of Figure $6.3(-)$ and the simplified model of Figure $6.5(--)$ are shown.

and $\sigma=0.16$. Matching this advance ratio and cavitation number required only a small extrapolation of the data in Figure 5.2, yielding values of $K^{*}=4.5 \times 10^{-6} \mathrm{~ms}^{2}$ and $M^{*}=4.5 \times 10^{-4} \mathrm{~s}$ at $n=28.3 \mathrm{~Hz}$. The calculations of Figure 5.3, however, extend only to an advance ratio of $J=0.9$, requiring a more uncertain extrapolation to obtain values of $K^{*}=10 \times 10^{-6} \mathrm{~ms}^{2}$ and $M^{*}=10 \times 10^{-4} \mathrm{~s}$ at $n=31.7 \mathrm{~Hz}$. Finally, interpolating between these values to the appropriate propeller rotation speed gave the values of $K^{*}=5.9 \times 10^{-6} \mathrm{~ms}^{2}$ and $M^{*}=5.9 \times 10^{-4} s$ used in producing Figure 6.4. As suggested in Section 4.2.3, the tunnel compliance was chosen to be $C_{t}=5 \times 10^{-3} \mathrm{~ms}^{2}$, a value providing the correct system natural frequency determined in the varying overflow tank pressure experiments of Figure 4.10. The estimates of Table 4.3 were used to evaluate the 
remaining parameters, though the resistive effects have initially been omitted from the calculation.

Immediately apparent from Figure 6.4 is that the real part of the system impedance is negative over two frequency ranges centered at approximately $f=0.12 \mathrm{~Hz}$ and $f=$ $25 \mathrm{~Hz}$. The cavitation dynamics discussed in Section 6.1 are still evident, but with the addition of the facility dynamics, the positive activity has been limited to these two frequency ranges. The trough at $f=0.12 \mathrm{~Hz}$ corresponds to the natural frequency discussed in Section 4.2.2. The particular value of $f=0.12 \mathrm{~Hz}$ can be confirmed in Figure 4.10 given the overflow tank pressure of $\bar{p}_{o t}=18.2 \mathrm{kPa}$ associated with the reduced test section pressure during the instability experiments.

Most importantly, the trough at $f=25 \mathrm{~Hz}$ seems a probable explanation for the propeller instability. At this frequency, the combination of facility and cavitation dynamics results in the creation of fluctuation energy and therefore potentially unstable behavior of the cavitation on the propeller. However, there is a large discrepancy between the frequency of this trough and the typical experimentally observed instability frequency of $f=10 \mathrm{~Hz}$.

\subsubsection{Simplified Model}

Further insight into the behavior of the combined facility and cavitation dynamics systems near this trough can be gained by considering the behavior of the simplified system shown in Figure 6.5, where the overflow tank and its connecting pipe have been removed from the system. Mathematically, this corresponds to assuming the impedance $Z_{c}$ of Equation 6.13 to be infinite, thereby removing the pole of the system impedance associated with the connecting pipe and overflow tank behavior. As demonstrated in Figure 6.4, which also includes the system impedance based on this simplified network, the simplification has no significant impact on the behavior of the system at higher frequencies. The resulting 


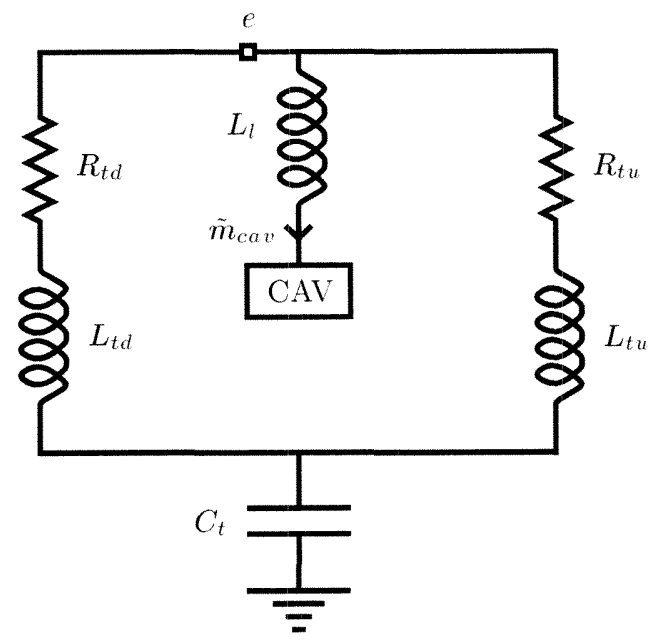

Figure 6.5: Schematic of simplified facility and cavitation dynamics.

algebraic expression for the system impedance at point $e$ is greatly simplified, with

$$
Z=\frac{L_{t} C_{t} \omega^{2}-1}{j \omega\left[L_{t} C_{t} K^{*} \omega^{2}+j\left[\frac{L_{t}}{L_{t u}}\right] C_{t} M^{*} \omega-\left(C_{t}+K^{*}\right)\right]},
$$

where once again $L_{t}^{-1}=L_{t u}{ }^{-1}+L_{t d}{ }^{-1}$.

The non-zero positive poles of this impedance are complex due to the presence of the term in the denominator associated with the mass flow gain factor. However, this term is rather small compared to the other two terms of the denominator, and thus a reasonable approximation of the pole location is

$$
\omega_{o}=\left[\frac{C_{t}+K^{*}}{L_{t} C_{t} K^{*}}\right]^{\frac{1}{2}}
$$

Further, since $C_{t} \gg K^{*}$, the pole is essentially determined by

$$
\omega_{o}=\left[\frac{1}{L_{t} K^{*}}\right]^{\frac{1}{2}}
$$

The location of this pole is also the location of the trough in the real part of the system 
impedance shown in Figure 6.4. Evaluating Equation 6.17 yields a frequency of $f=$ $25.0 \mathrm{~Hz}$. In comparison, the exact frequency of the trough in Figure 6.4 is $f=25.2 \mathrm{~Hz}$, indicating that the approximation is quite accurate.

As an aside, this analysis can be applied to cavitating pumps to help explain the experimental observations of Brennen (1994) that the auto-oscillation frequency for many

different impellers is roughly proportional to $\sigma^{\frac{1}{2}}$. It is clear from Equation 6.17 that since $K^{*}$ is roughly inversely proportional to $\sigma$ (Brennen, Meissner, Lo, and Hoffman 1982), the predicted auto-oscillation frequency will be proportional to $\sigma^{\frac{1}{2}}$.

Equation 6.17 reveals that the location of the trough in the real part of the system impedance is not dependent on the value chosen for the tunnel compliance, $C_{t}$. This is fortunate given the difficulties encountered in accurately estimating this parameter. The location of the trough is, however, dependent on the value of the cavitation compliance, and this parameter is also not known with great precision. As noted in Section 5.2, the values obtained from the stable propeller estimates are only quasistatic estimates, while the instability is a dynamic phenomenon. Moreover, a substantial extrapolation of this data was required in Section 6.3.1 to obtain an estimate of this parameter at the instability conditions. Finally, and perhaps most importantly, there are the possible effects of tip vortex participation in the volumetric fluctuations during the instability cycle.

\subsection{Effect of Tip Vortex Participation}

As discussed in Section 5.4, the participation of the tip vortices in the volumetric fluctuations would increase the cavitation compliance. This would reduce the predicted instability frequency, $\omega_{o}$, perhaps closer to the observed value. Figure 6.6 shows the effect of increasing the cavitation compliance on the real part of the system impedance calculated for the simplified system of Figure 6.5. As anticipated by Equation 6.17, the location of the trough moves to lower frequencies. A factor of six increase in the compliance, as estimated in Section 5.4, results in a trough at a frequency of $f=10.4 \mathrm{~Hz}$. This indicates that including the effect of tip vortex participation can bring the instability frequency predicted by the combined facility and cavitation dynamics model into good agreement 


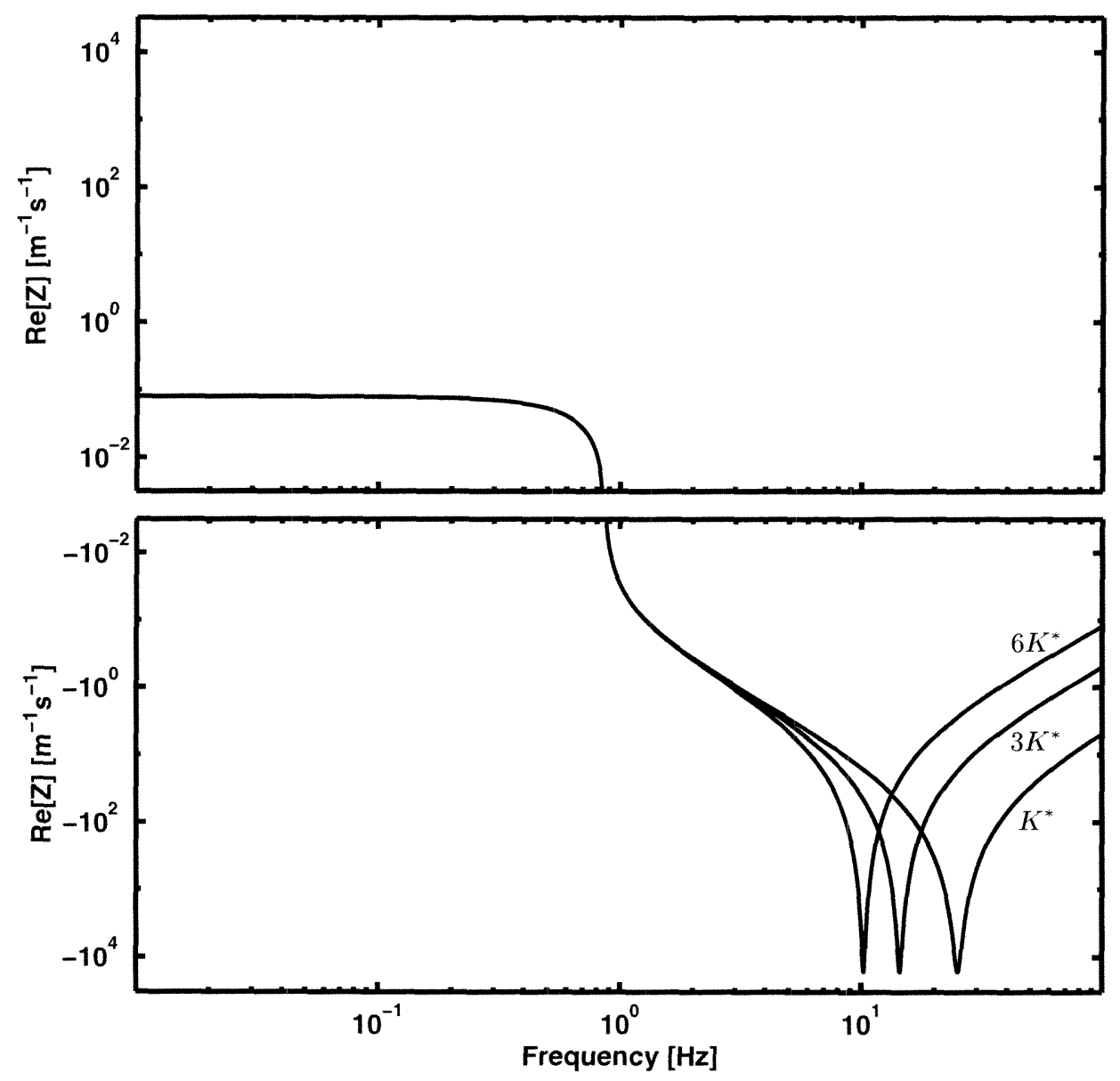

Figure 6.6: Real part of system impedance for various cavitation compliances. Three curves are shown for cavitation compliance values of one, three, and six times the value in Figure 6.4.

with the experimentally observed frequency.

\subsection{Variation of the Instability Frequency}

As discussed in Section 3.3.7, the Strouhal frequency did not adequately predict the variations in instability frequency seen with both varying advance ratio and cavitation number. This suggested that a mechanism other than vortex shedding was responsible for determining the frequency of the instability.

Inspection of Figures 5.2 and 5.3 reveals that the cavitation compliance decreases with increasing advance ratio. Based on Equation 6.17 and as illustrated in Figure 6.6, 
this results in a higher instability frequency. This trend is consistent with that of Figure 3.17 , where the experimentally observed instability frequency does indeed increase with increasing advance ratio. Also, the cavitation compliance decreases with increasing cavitation number, again resulting in a higher instability frequency. Unlike the Strouhal frequency, this variation does offer an explanation of the trend seen in Figure 3.18, where the instability frequency is found to increase with increasing cavitation number. These correspondences strongly support the notion that the instability frequency is in fact determined by the location of the trough in the real part of the system impedance.

It is also worth noting here, as in Section 3.3.6, that the observed instability frequency did not change with increasing instability intensity. This provides some evidence that although the instability is surely a highly non-linear phenomenon, the linear model developed here is adequate in predicting not only the frequency of the instability at onset but at greater intensities as well.

\subsection{Effect of Resistance}

The analyses of Sections 6.3.1 and 6.3.2 did not include any system resistances. Not surprisingly, inclusion of these resistances tends to increase the real part of the impedance at all frequencies, but these effects are especially dramatic and also more complex near the trough detailed in Section 6.3.2. Figure 6.7 shows the real part of the system impedance for various values of the tunnel resistances. Here, the upstream and downstream resistances, $R_{t u}$ and $R_{t d}$, are assumed equal, and the cavitation compliance is evaluated including the component contributed by the tip vortex cavitation. The system impedance is calculated for several values of the non-dimensional damping parameter,

$$
\zeta=\frac{5 R_{t} K^{*}}{3 M^{*}}
$$

where $R_{t}^{-1}=R_{t u}^{-1}+R_{t d}^{-1}$ is an effective resistance based on the parallel combination of the upstream and downstream resistances. This parameter represents a ratio of the resistive effects opposing unstable behavior to the dynamic characteristic driving the instability, the quantity $\frac{M^{*}}{K^{*}}$. 


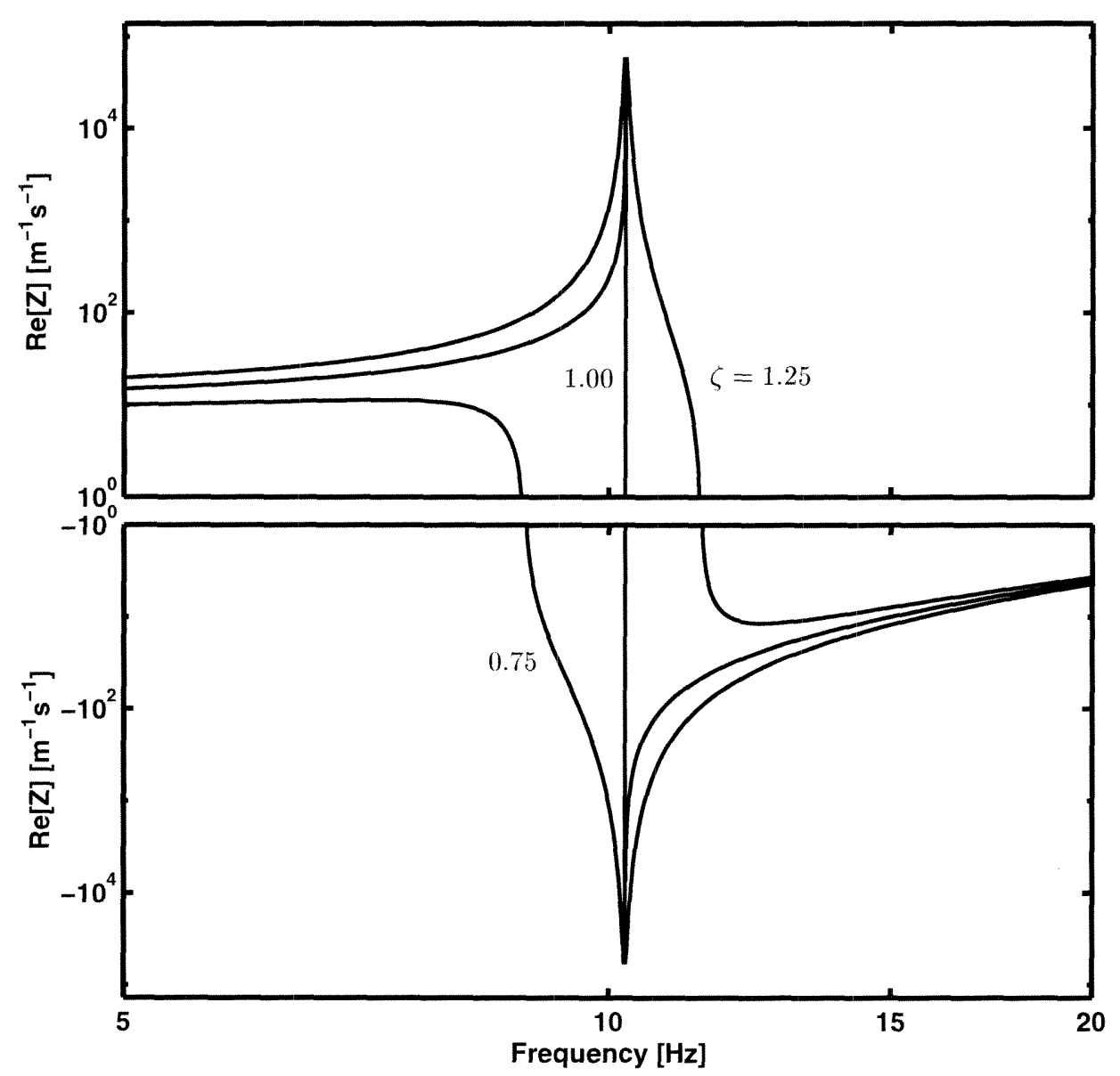

Figure 6.7: Effect of resistance on real part of system impedance. The real part of the system impedance is shown for several values of the parameter $\zeta$, including the critical value of $\zeta=1$, with $R_{t u}=R_{t d}$.

It is apparent from Figure 6.7 that the system impedance undergoes a dramatic transition at $\zeta=1$. The well defined trough transforms into a dramatic positive peak in the real part of the system impedance. At the value of $\zeta=1.25$, the real part of the system impedance does regain negative values at higher frequencies, but the amplitude of these negative values is several orders of magnitude lower than those of the trough present when $\zeta<1$. For sufficiently high values of $\zeta$, the real part of the system impedance remains positive across all frequencies.

The tunnel resistance estimate of Table 4.3, obtained initially from tunnel geometry and then revised based on the experimentally determined facility response, results in a damping value of $\zeta=2.0$. Based on this estimate, it would seem the facility has 
sufficient resistance to effectively damp out any potentially unstable behavior. However, this estimate of $\zeta$ is based on a cavitation compliance including the effects of tip vortex participation. If these effects are not considered in estimating the cavitation compliance, a value of $\zeta=0.33$ is obtained. As shown in Section 6.4, though, this reduced value of the cavitation compliance does not correctly predict the experimentally observed instability frequency. The value of $\zeta$ would also be lowered by an increased value of the mass flow gain factor. As noted in Section 5.4, this parameter may be larger than the estimate used here due to the effects of tip vortex participation. Finally, it is possible that the value of the tunnel resistance estimated in Table 4.3 is too large.

If the upstream and downstream resistances are not assumed equal, the behavior of the system impedance becomes significantly more complicated. Figure 6.8 shows the real part of the system impedance at various ratios of upstream to downstream tunnel resistance. Despite the fact that all three curves are generated for a damping value of $\zeta=1.0$, the case of a lesser upstream resistance results in unstable behavior. In contrast, a greater upstream resistance results in stable behavior. Thus, the model predicts that unstable behavior is favored by a relatively smaller upstream resistance. This directly contradicts laboratory observations, where the presence of the gear case upstream of the propeller, which presumably increases the upstream resistance, was necessary for observation of the instability.

\subsection{Modeling of the Far Field Pressure}

Section 3.3.8 demonstrates that a model incorporating only the fluctuating cavity volume is inadequate for predicting the pressures generated by the instability and that a model incorporating the facility dynamics is needed to explain the dramatic pressure fluctuations that were observed experimentally. Such a model was developed in Chapter 4, with the resulting system impedance, including the effect of the estimated resistances, shown in Figure 4.19. This model yields a magnitude of the system impedance of $|Z|=354 \mathrm{~m}^{-1} \mathrm{~s}^{-1}$ at the instability of frequency of $f=10 \mathrm{~Hz}$. Based on Equations 4.1 and 4.4 , it is clear 


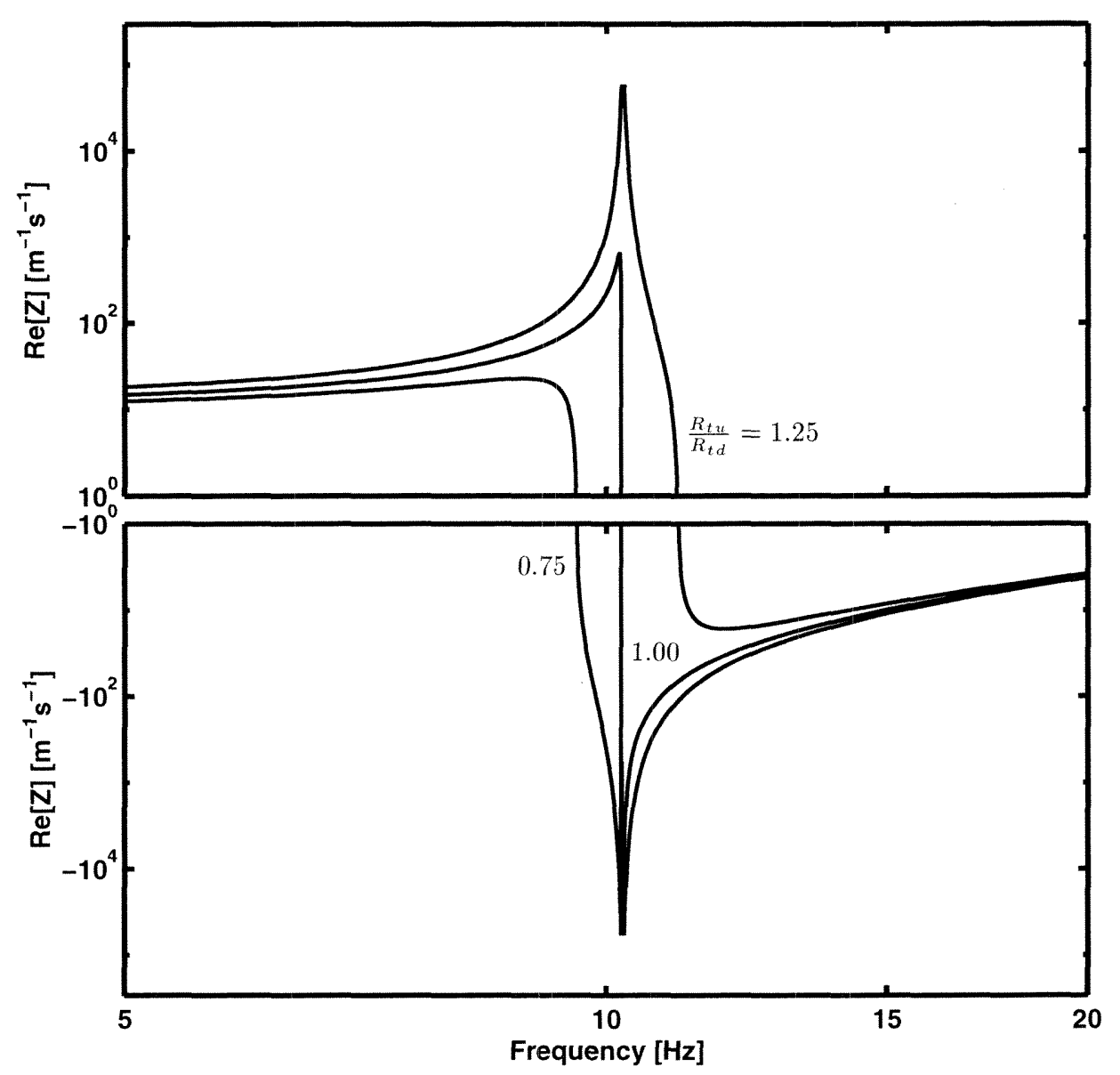

Figure 6.8: Effect of unbalanced upstream and downstream resistances. The real part of the system impedance is shown for several values of $\frac{R_{t u}}{R_{t d}}$, with $\zeta=1.0$.

that

$$
\left|\tilde{p_{e}}\right|=\rho \omega\left|\tilde{V}_{c a v}\right||Z|
$$

yielding a fluctuating pressure amplitude of $\left|\tilde{p}_{e}\right|=934 \mathrm{~Pa}$. This is significantly lower than the observed amplitudes, which were as high as $\left|\tilde{p}_{e}\right|=10 \mathrm{kPa}$.

However, as demonstrated, the inclusion of the cavitation dynamics in combination with the facility dynamics yields a very different system impedance. Shown in Figure 6.9 is the magnitude of the system impedance for the system of Figure 6.3. Easily distinguished is the peak in the impedance at a frequency of $f=10.4 \mathrm{~Hz}$. Of course, the exact magnitude of the impedance at this frequency, and therefore the predicted ampli- 


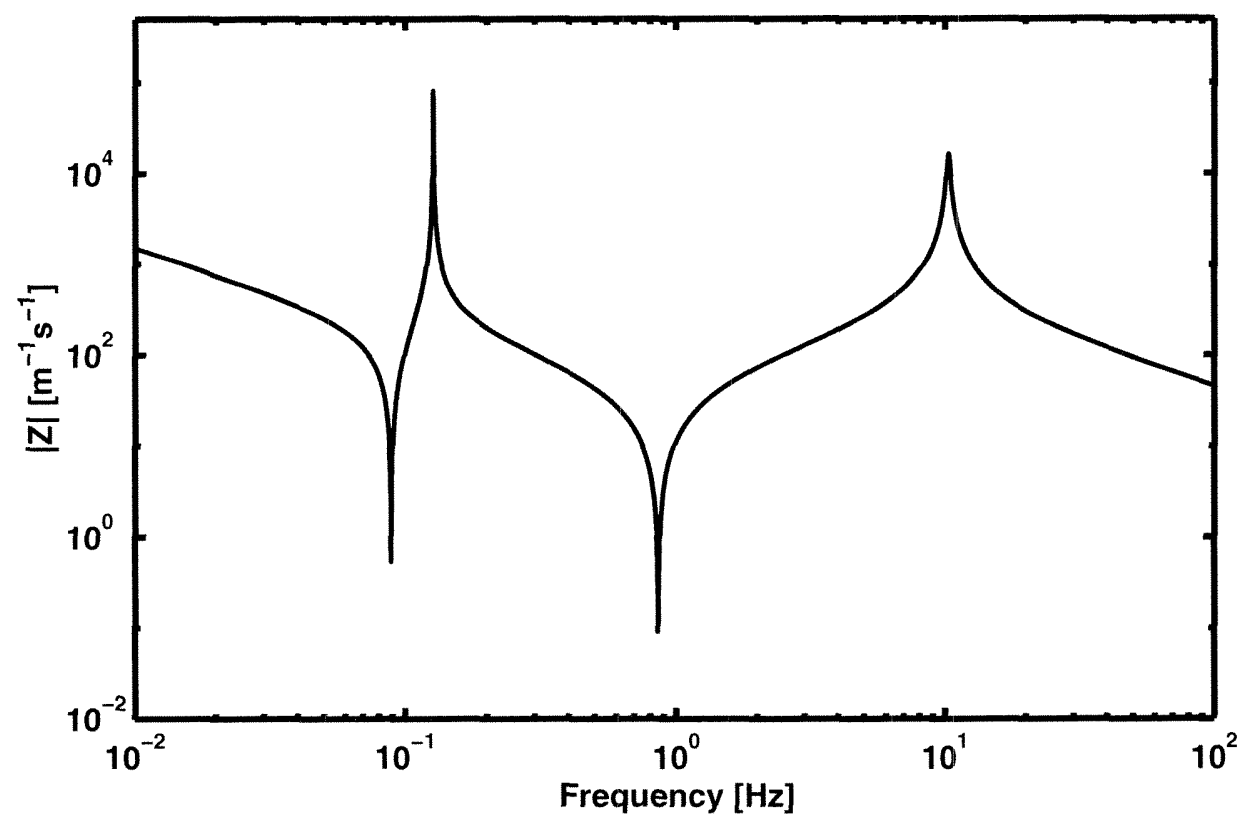

Figure 6.9: Magnitude of system impedance for combined dynamics model. $|Z|$ is shown for the system of Figure 6.3 .

tude of the fluctuating pressures, will be determined by the value of the damping factor $\zeta$. The difficulties encountered in estimating all three parameters affecting $\zeta$ make a numerical comparison of the predicted and observed fluctuating pressure amplitudes difficult. However, a value of $|Z|=1 \times 10^{4} \mathrm{~m}^{-1} \mathrm{~s}^{-1}$ can be estimated from Figure 6.9 , yielding a fluctuating pressure amplitude of $\left|\tilde{p}_{e}\right|=26 \mathrm{kPa}$. This is somewhat greater than the $10 \mathrm{kPa}$ amplitude observed experimentally, a fact that is most likely attributable to non-linear resistances not represented in the linear model. It is nonetheless clear that the combined facility and cavitation dynamics model provides a magnitude of the system impedance capable of yielding the very dramatic amplitudes that were observed experimentally. 


\section{Chapter 7}

\section{Conclusions}

The current research effort has proven successful in identifying and describing a previously unreported surge instability on a cavitating model propeller. The dynamics of the instability were investigated with a special effort to separate propeller cavitation dynamics and the dynamics of the surrounding facility. The uncertainty in estimating many of the parameters encountered places limits on detailed characterization of the model. Nonetheless, the work provides many valuable conclusions and suggests topics for future study.

\subsection{Summary of Thesis Work}

Summarized below are the principal results and conclusions presented in the current work.

- The stable cavitating performance of the propeller exhibited dependence not only on the advance ratio and cavitation number but also on the propeller rotation speed. The cavitation extent increased with decreasing advance ratio and cavitation number. However, with these quantities constant, substantial variations in the cavitation extent were observed with changing propeller rotation speed.

- The behavior of the propeller at yaw indicated that the cavitation extent closely tracked the effective angle of attack of the propeller blades. The variation in cavitation extent with angular location during operation at yaw was consistent with the quasistatic predictions of an effective angle of attack calculation. In the case of the 
highest yaw angles explored, variations in excess of that predicted by the effective angle of attack suggested the possibility of additional cavitation dynamics.

- A previously unseen cavitation surge instability was observed with the propeller operating downstream of the supporting gear case. The instability was characterized by a periodic fluctuation in the cavitation extent both on the propeller blades and in the tip vortices. The noise generated by the instability was dominated by a single low frequency, typically around $f=10 \mathrm{~Hz}$. This matched the visually observed frequency of the fluctuation in cavity volume.

- The instability showed re-entrant jet behavior characteristic of three-dimensional swept hydrofoils. In particular, the nature of cavity closure inboard and outboard of the point of re-entrant jet impingement on the leading edge was similar to that reported by previous investigators.

- The general nature, onset, and reduced frequency of the instability were similar to partial cavity instability observations on two-dimensional hydrofoils. The reduced frequency calculated for the propeller instability showed reasonable agreement to that reported in previous hydrofoil studies. The onset of the propeller instability, like the partial cavity instability, appeared closely correlated to the ratio of the cavity length on a propeller blade to the blade chord.

- The measured fluctuating pressures correspond closely to the volumetric acceleration imposed upon the flow. The volumetric acceleration, calculated from cavity length measurements over an instability cycle, tracked the frequency, phase, and shape of the pressure measured on the test section floor.

- A model developed for the facility dynamics offered good agreement with the experimentally measured dynamics of the facility. The predictions of a lumped parameter model incorporating resistive, inertive, and compliant behaviors closely matched the observed natural frequency and forced response of the facility. The proposed model illustrated the importance of the tunnel compliance in determining the facility dynamics. 
- Estimates of the cavitation compliance and mass flow gain factor suggested that the inclusion of the cavity dynamics in a comprehensive system model would result in active system dynamics. Using cavitation extent measurements made during stable propeller operation, quasistatic estimates were obtained for the cavitation compliance and mass flow gain factor. The values obtained offered reasonable agreement with those obtained in investigations of cavitating pump instabilities. A system containing only the cavitation dynamics was characterized by a negative real part of the system impedance. This implied an increasing fluctuation energy and therefore unstable system behavior.

- A comprehensive model incorporating both the facility and cavitation dynamics predicted the unstable cavitating behavior. The model exhibited a negative real part of the system impedance across narrow frequency ranges centered about two distinct frequencies. It was hypothesized that one of these frequencies corresponded to the observed unstable cavitating behavior.

- The predicted instability frequency was highly dependent on the estimated value of the cavitation compliance. The frequency predicted with the initial estimate of the cavitation compliance was significantly higher than the observed instability frequency. Inclusion of the fluctuating tip vortex volume in the cavitation compliance estimate resulted in excellent agreement between the predicted and observed frequencies.

- The model offered insight into the variation of the instability frequency. The predicted variations in instability frequency with cavitation number and advance ratio matched the experimentally observed trends. In contrast, these variations were not fully explained by an analysis hypothesizing vortex shedding as the determining factor of the instability frequency.

- The model predicted that the instability will not occur above a critical value of the tunnel resistance. Above this critical value of the tunnel resistance, the negative real part of the system impedance became positive, and the system dynamics therefore transitioned from active to inactive. 
- The model does not explain why the instability only occurs with the propeller mounted downstream of the gear case. The model more strongly favored unstable behavior when the downstream resistance exceeds the upstream resistance. This implied that the instability should have occurred with the propeller mounted upstream of the added resistance of the supporting gear case. This was in direct contradiction with experimental observations. It remained possible that the instability was triggered, but not sustained, by irregularities in the gear case wake.

- It could not be established that the linear resistive lumped parameters adequately captured the resistive behavior of the facility. Moreover, the precise value of the linearized resistances could not be determined precisely. Thus, the validity of predictions based on resistive behaviors remained uncertain.

- The peak in the system impedance at the predicted instability frequency adequately predicts the large amplitude of the fluctuating pressures observed during the instability. The added resonance associated with the instability more closely matched the observed pressure fluctuations than a model incorporating only the facility dynamics.

\subsection{Future Research}

The remaining uncertainty in several of the results and findings detailed above suggests several topics for future investigations. First, although the lumped parameter model utilized in the current work proved adequate in most respects, it is possible that a distributed parameter approach would offer more insight into the nature of the facility dynamics. This would also allow for more precise representation of the tunnel compliance. In addition, efforts should be made to better understand the effects on the tunnel compliance of trapped and dissolved air within the facility. Finally, it is possible that the resistive behavior of the facility could be more accurately determined through the use of non-linear resistance models.

It remains unclear after the present analysis whether or not a similar instability will occur in the environment downstream of a ship hull. The current work only reveals 
that the likelihood of an instability is strongly dependent on the nature of the dynamics surrounding the cavitating device. A natural extension of the work, then, would be a study to determine the cavitation dynamic parameters and a model for the dynamics in the wake of a ship hull.

More generally, the present work is a testament to the importance of facility dynamics in determining the nature of dynamic cavitation phenomena. Future investigators of such phenomena should take care to consider these effects. In particular, it is necessary to determine if the cavitating device is characterized by cavitation dynamic parameters that could potentially lead to active system dynamics. Only then can conclusions be drawn regarding the true origins of an observed dynamic cavitation phenomenon. 


\section{Bibliography}

Bark, G. (1986). Development of violent collapses in propeller cavitation. In Proc. Cavitation and Multiphase Flow Forum, pp. 65-76. ASME.

Bark, G. and van Berlekom, W. B. (1978). Experimental investigations of cavitation noise. In Proc. 12th ONR Symp. on Naval Hydrodynamics, pp. 470-493.

Blake, W. K. (1986). Propeller cavitation noise: the problems of scaling and prediction. In Proc. Int. Symp. on Cavitation and Multiphase Flow Noise, Volume FED 45, pp. 89-100. ASME.

Braisted, D. M. and Brennen, C. E. (1978). Observations on instabilities of cavitating inducers. In Proc. Cavitation and Polyphase Flow Forum, pp. 19-22. ASME.

Braisted, D. M. and Brennen, C. E. (1980). Auto-oscillation of cavitating inducers. In Proc. Symp. on Polyphase Flow and Transport Tech., pp. 157-166. ASME.

Brennen, C. E. (1978). Bubbly flow model for the dynamic characteristics of cavitating pumps. J. Fluid Mech. 89, 223-240.

Brennen, C. E. (1994). Hydrodynamics of Pumps. Concepts ETI and Oxford Univ. Press.

Brennen, C. E. (1995). Cavitation and Bubble Dynamics. Oxford Univ. Press.

Brennen, C. E. and Acosta, A. J. (1973). Theoretical, quasistatic analyses of cavitation compliance in turbopumps. J. Spacecraft and Rockets 10(3), 175-180.

Brennen, C. E. and Acosta, A. J. (1976). The dynamic transfer function for a cavitating inducer. ASME J. Fluids Eng. 98, Part 2, 182-191.

Brennen, C. E., Meissner, C., Lo, E. Y., and Hoffman, G. S. (1982). Scale effects in 
the dynamic transfer functions for cavitating inducers. ASME J. Fluids Eng. 104, 428-433.

d'Agostino, L. and Brennen, C. E. (1983). On the acoustical dynamics of bubble clouds. In Proc. Cavitation and Multiphase Flow Forum, pp. 72-75. ASME.

d'Agostino, L. and Brennen, C. E. (1989). Linearized dynamics of spherical bubble clouds. J. Fluid Mech. 199, 155-176.

de Lange, D. F., de Bruin, G. J., and van Wijngaarden, L. (1994). On the mechanism of cloud cavitation - experiment and modeling. In Proc. 2nd Int. Symp. on Cavitation, Tokyo, pp. 45-50.

Franc, J. P. and Michel, J. M. (1988). Unsteady attached cavitation on an oscillating hydrofoil. J. Fluid Mech. 193, 171-189.

Gates, E. M. (1977). The influence of freestream turbulence, freestream nuclei populations, and drag-reducing polymer on cavitation inception on two axisymmetric bodies. Technical Report E182-2, California Institute of Technology.

Hart, D. P. (1993). Cavitation and Wake Structure of Unsteady Tip Vortex Flows. Ph.D. thesis, California Institute of Technology.

Hart, D. P., Brennen, C. E., and Acosta, A. J. (1990). Observations of cavitation on a three-dimensional oscillating hydrofoil. In Proc. Cavitation and Multiphase Flow Forum, Volume FED 98, pp. 49-52. ASME.

Hennyey, Z. (1962). Linear Electric Circuits. Addison-Wesley Publ., Inc.

Huse, E. (1972). Pressure fluctuations on the hull induced by cavitating propellers. Technical Report 111, Norwegian Ship Model Experiments Tank.

Jessup, S. (1997). Cavitation on the 4990 hydrofoil. Private communication.

Kjeldsen, M., Vennatro, R., Arndt, R., and Keller, A. (1999). Discussion on cyclic cavitation in closed water tunnels and the influence from the dynamic response of the tunnel. In Proc. Behavior of Hyd. Machinery Under Steady Oscillatory Conditions Work Group. IAHR. 
Knapp, R. T. (1955). Recent investigations of the mechanics of cavitation and cavitation damage. Trans. ASME 77, 1045-1054.

Kubota, A., Kato, H., Yamaguchi, H., and Maeda, M. (1989). Unsteady structure measurement of cloud cavitation on a foil section using conditional sampling. ASME J. Fluids Eng. 111, 204-210.

Laberteaux, K. and Ceccio, S. (1998). Partial attached cavitation on two- and threedimensional hydrofoils. In Proc. 22nd ONR Symp. on Naval Hydrodynamics, pp. 239254.

Le, Q., Franc, J. P., and Michel, J. M. (1989). Partial cavities: global behaviour and mean pressure distribution. ASME J. Fluids Eng. 115, 243-248.

Liu, Z. (1995). Nuclei Population Dynamics and Cavitation. Ph.D. thesis, California Institute of Technology.

McKenney, E. (1995). A Study of Tip Vortices and Cavitation on a Propeller in a Non-uniform Flow Field. Ph.D. thesis, California Institute of Technology.

McKenney, E. A. and Brennen, C. E. (1994). On the dynamics and acoustics of cloud cavitation on an oscillating hydrofoil. In Proc. Symp. on Cavitation and Gas-Liquid Flows in Fluid Machinery and Devices, Volume FED 190, pp. 195-202. ASME.

Munson, B. R., Young, D. F., and Okiishi, T. H. (1994). Fundamentals of Fluid Mechanics. John Wiley and Sons, Inc.

Ng, S. L. and Brennen, C. E. (1978). Experiments on the dynamic behavior of cavitating pumps. Trans. ASME 100, 166-176.

Otsuka, S., Tsujimoto, Y., Kamijo, K., and Furuya, O. (1996). Frequency dependence of mass flow gain factor and cavitation compliance of cavitating inducers. ASME J. Fluids Eng. 118, 400-408.

Reisman, G. E. (1997). Dynamics, Acoustics and Control of Cloud Cavitation on Hydrofoils. Ph.D. thesis, California Institute of Technology.

Reisman, G. E., Wang, Y.-C., and Brennen, C. E. (1998). Observations of shock waves in cloud cavitation. J. Fluid Mech. 355, 255-283. 
Shen, Y. and Peterson, F. B. (1978). Unsteady cavitation on an oscillating hydrofoil. In Proc. 12th ONR Symp. on Naval Hydrodynamics, pp. 362-384.

Soyama, H., Kato, H., and Oba, R. (1992). Cavitation observations of severely erosive vortex cavitation arising in a centrifugal pump. In Proc. 3rd Int. Mech. Eng. Conf. on Cavitation, pp. 103-110.

Tsujimoto, Y., Kamijo, K., and Yoshida, Y. (1993). A theoretical analysis of rotating cavitation in inducers. ASME J. Fluids Eng. 115, 135-141.

Tulin, M. P. (1953). Steady two-dimensional cavity flows about slender bodies. Technical Report 834, David Taylor Model Basin.

van Wijngaarden, L. (1964). On the collective collapse of a large number of gas bubbles in water. In Proc. 11th Int. Conf. Appl. Mech., pp. 854-861. Springer-Verlag.

Wade, R. B. and Acosta, A. J. (1966). Experimental observations on the flow past a plano-convex hydrofoil. ASME J. Basic Eng. 88, 273-283.

Weitendorf, E.-A. (1989). 25 years research on propeller excited pressure fluctuations and cavitation. In Proc. Int. Symp. on Cavitation Noise and Erosion in Fluid Systems, Volume FED 18, pp. 1-10. ASME. 